- ofo

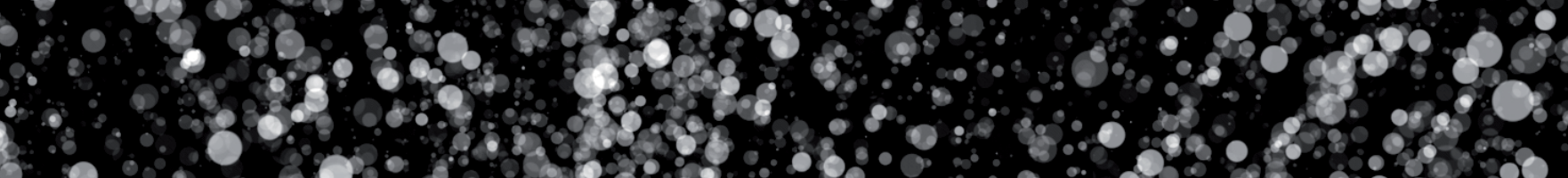

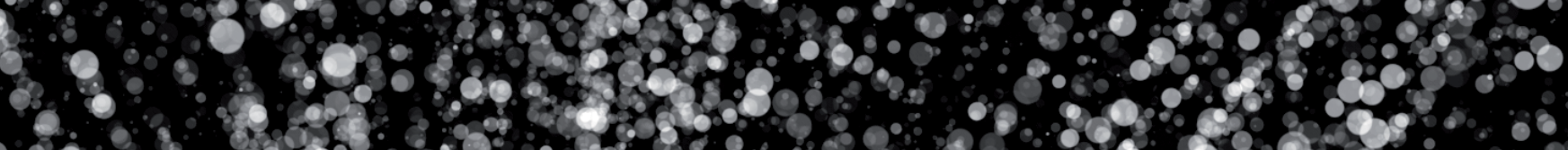

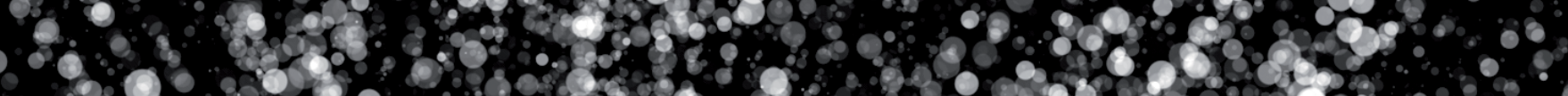

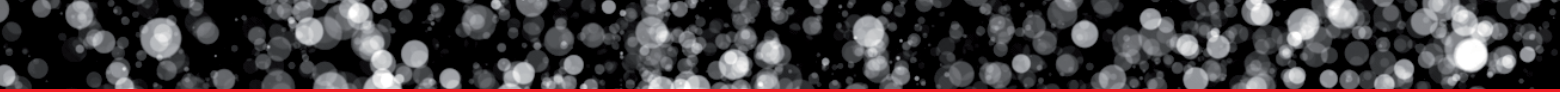

\title{
IntechOpen
}

\section{Single Photon Manipulation}

Edited by Keyu Xia

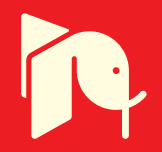

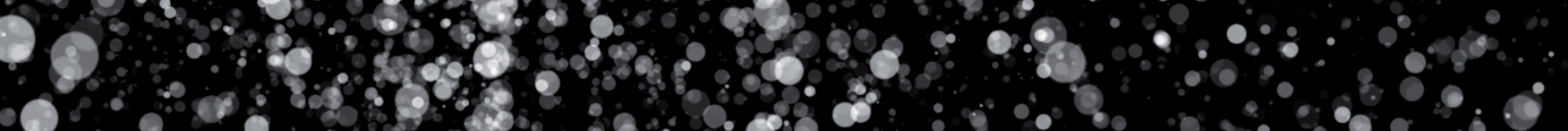

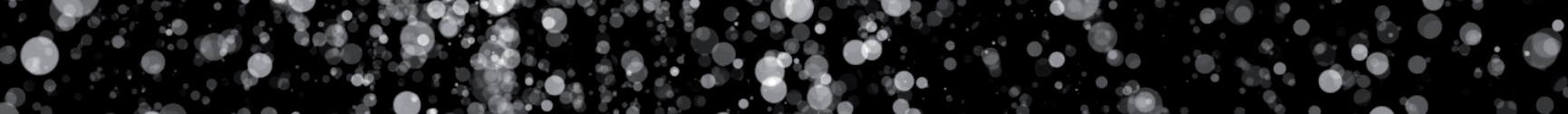

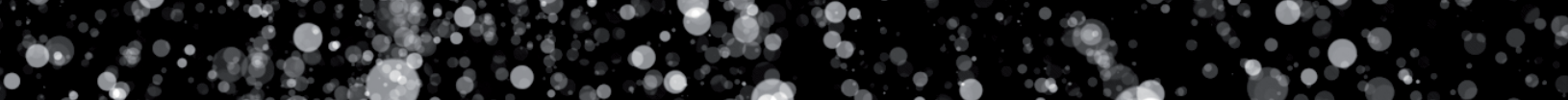

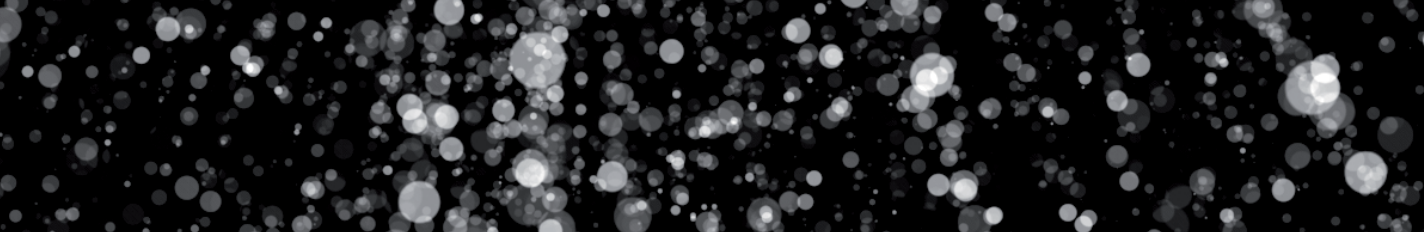

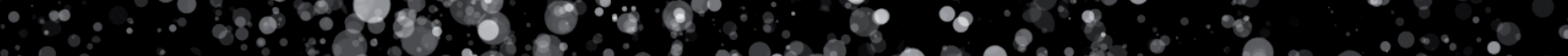





\section{Single Photon Manipulation \\ Edited by Keyu Xia}



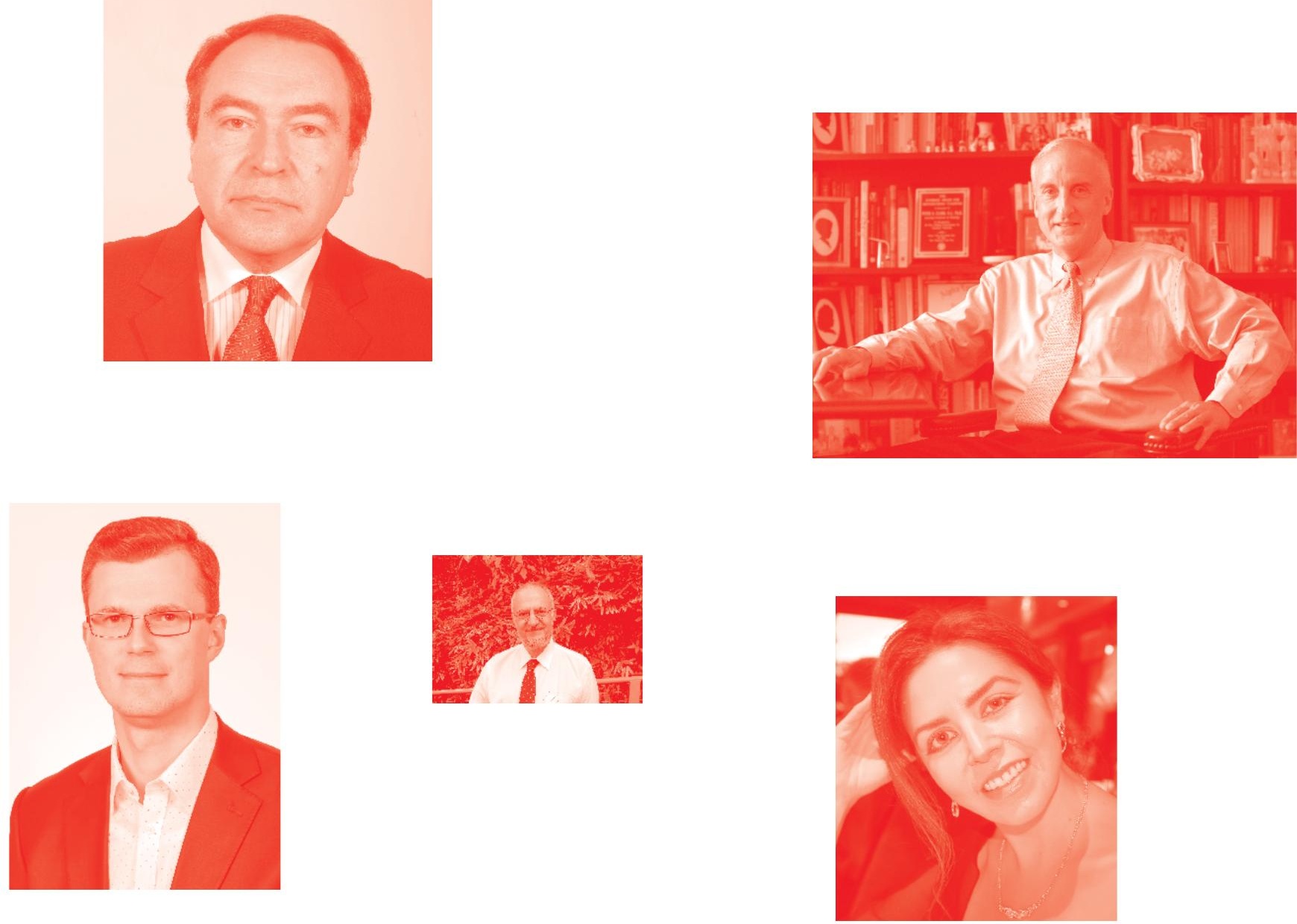

Supporting open minds since 2005
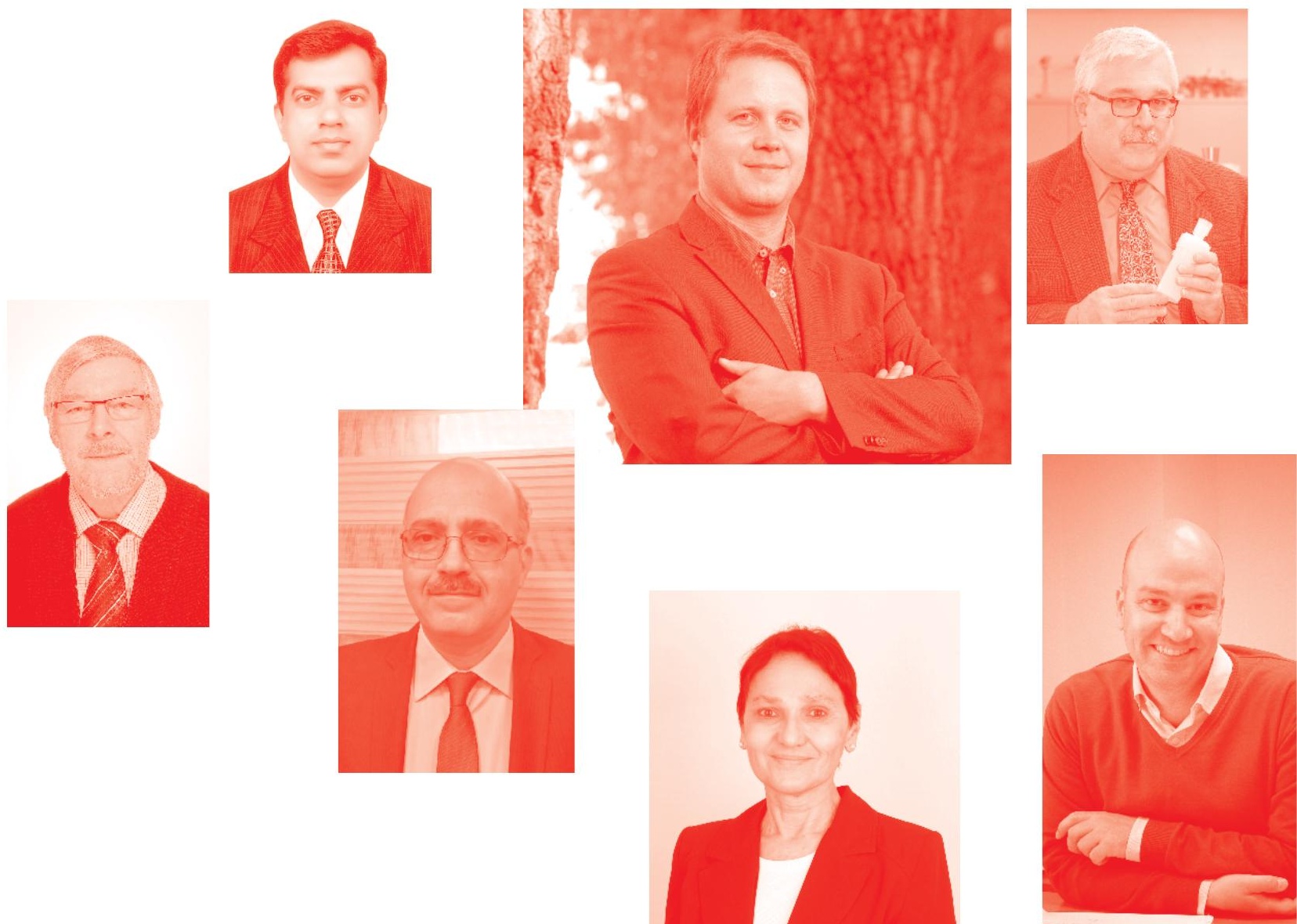
Single Photon Manipulation

http : //dx. doi . org/10.5772/intechopen. 83207

Edited by Keyu Xia

Contributors

Constantin Meis, Chandrasekhar Roychoudhuri, Yuanhua Li, Xianfeng Chen, Lei Tang, Keyu Xia, Zhi-Yuan Zhou, Bao-Sen Shi

๑) The Editor(s) and the Author(s) 2020

The rights of the editor(s) and the author(s) have been asserted in accordance with the Copyright. Designs and Patents Act 1988. All rights to the book as a whole are reserved by INTECHOPEN LIMITED. The book as a whole (compilation) cannot be reproduced, distributed or used for commercial or non-commercial purposes without INTECHOPEN LIMITED's written permission. Enquiries concerning the use of the book should be directed to INTECHOPEN LIMITED rights and permissions department (permissions@intechopen.com).

Violations are liable to prosecution under the governing Copyright Law .

\section{(c) BY}

Individual chapters of this publication are distributed under the terms of the Creative Commons Attribution 3. 0 Unported License which permits commercial use, distribution and reproduction of the individual chapters, provided the original author(s) and source publication are appropriately acknowledged. If so indicated, certain images may not be included under the Creative Commons license. In such cases users will need to obtain permission from the license holder to reproduce the material. More details and guidelines concerning content reuse and adaptation can be found at http : //www . intechopen. com/copyright-policy. html.

Notice

Statements and opinions expressed in the chapters are these of the individual contributors and not necessarily those of the editors or publisher. No responsibility is accepted for the accuracy of information contained in the published chapters. The publisher assumes no responsibility for any damage or injury to persons or property arising out of the use of any materials, instructions, methods or ideas contained in the book.

First published in London, United Kingdom, 2020 by IntechOpen

IntechOpen is the global imprint of INTECHOPEN LIMITED, registered in England and Wales, registration number: 11086078 , 5 Princes Gate Court, London, SW7 2QJ, United Kingdom Printed in Croatia

British Library Cataloguing-in-Publication Data

A catalogue record for this book is available from the British Library

Additional hard and PDF copies can be obtained from orders@intechopen.com

Single Photon Manipulation

Edited by Keyu Xia

p. cm.

Print ISBN 978-1-83880-353-7

Online ISBN 978-1-83880-354-4

eBook (PDF) ISBN 978-1-83880-606-4 


\section{We are IntechOpen, \\ the world's leading publisher of Open Access books}

\section{Built by scientists, for scientists}

\section{$5,100+$}

Open access books available

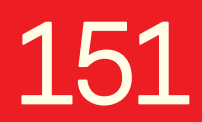

Countries delivered to

\author{
$126,000+$ \\ International authors and editors
}

Our authors are among the

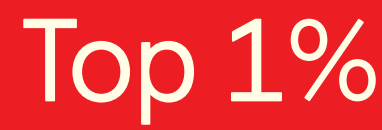

most cited scientists

Contributors from top 500 universities

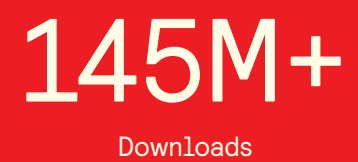

Downloads

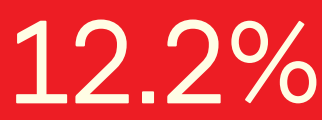

in Web of Science ${ }^{\mathrm{TM}}$ Core Collection (BKCI)

\section{Interested in publishing with us? \\ Contact book.department@intechopen.com}

Numbers displayed above are based on latest data collected.

For more information visit www.intechopen.com 



\section{Meet the editor}

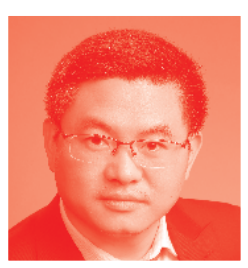

Keyu Xia was awarded his doctoral degree of "Optics" by the Shanghai Institute of Optics and Fine Mechanics, Chinese Academy of Sciences in 2007. He has been a full professor at Nanjing University since 2017. His research interest includes quantum optics and quantum information, in particular, chiral quantum optics. He has published more than 40 peer-reviewed publications. His academic achievements include: (I) proposing the first quantum-state-controlled photon isolator and circulator; (II) proposing a chiral cross-Kerr nonlinear medium to bypass the dynamical reciprocity in nonlinear isolation; (III) experimentally demonstrating the chiral susceptibility-momentum locking as a principle for optical nonreciprocity. Due to his significant contributions to quantum optics and quantum information technology, he was awarded the senior member designation of OSA in 2020. 



\section{Contents}

$\begin{array}{ll}\text { Preface } & \text { XIII }\end{array}$

Section 1 1

Basic Concept

Chapter 1

Do We Manipulate Photons or Diffractive EM Waves to Generate Structured Light?

by ChandraSekhar Roychoudhuri

Chapter 2

Quantized Field of Single Photons

by Constantin Meis

Section 2

Generation

Generation and Manipulation of Nonclassical Photon Sources

in Nonlinear Processes

by Zhi-Yuan Zhou and Bao-Sen Shi

Chapter 4

Single-Photon Frequency Conversion for Quantum Interface by Yuanhua Li and Xianfeng Chen

Section 3

Nonreciprocal Control

Chapter 5

Optical Chirality and Single-Photon Isolation

by Lei Tang and Keyu Xia 



\section{Preface}

This short book provides readers with some basic concepts of single photons and the latest developments in the field. The book reviews the most important method of generating single photons in experiments and it also introduces an emerging field of single-photon isolation.

The first section provides a very simple picture of the concept of evolution of the photon" from the classical to the quantum formalisms, and the quantization of the light field as a description of the photon. The next section focuses on recent experiments exploiting optical nonlinear processes to generate and manipulate single photons. In the last section, nonreciprocal quantum devices are designed to isolate the backscattering of single photons.

The book aims to present some basic knowledge of single photons to readers in a quick read but with not many details. 

Section 1

Basic Concept 



\title{
Do We Manipulate Photons or Diffractive EM Waves to Generate Structured Light?
}

\author{
ChandraSekhar Roychoudhuri
}

\begin{abstract}
In the domain of light emissions, quantum mechanics has been an immensely successful guiding tool for us. In the propagation of light and optical instrument design, Huygens-Fresnel diffraction integral (HFDI) (or its advanced versions) and Maxwell's wave equation are continuing to be the essential guiding tools for optical scientists and engineers. In fact, most branches of optical science and engineering, like optical instrument design, image processing, Fourier optics, Holography, etc., cannot exist without using the foundational postulates behind the Huygens-Fresnel diffraction integral. Further, the field of structured light is also growing where phases and the state of polarizations are manipulated usually with suitable classical macro-devices to create wave fronts that restructured through light-matter interactions through these devices. Mathematical modeling of generating such complex wave fronts generally follows classical concepts and classical macro tools of physical optics. Some of these complex light beams can impart mechanical angular momentum and spin-like properties to material particles inserted inside these structured beams because of their electromagnetic dipolar properties and/or structural anisotropy. Does that mean these newly structured beams have acquired new quantum properties without being generated through quantum devices and quantum transitions? In this chapter, we bridge the classical and quantum formalism by defining a hybrid photon (HP). HP is a quantum of energy, h $\nu$, at the initial moment of emission. It then immediately evolves into a classical time-finite wave packet, still transporting the original energy, $\mathrm{h} v$, with a classical carrier frequency $v$ (oscillation of the E-vector). This chapter will raise enquiring questions whether all these observed "quantum-like" behaviors are manifestations of the joint properties of interacting material particles with classical EM waves or are causal implications of the existence of propagation of "indivisible light quanta" with exotic properties like spin, angular momentum, etc.
\end{abstract}

Keywords: structured light, hybrid photon, non-interaction of light (NIW), Huygens principle, photoelectric effect, semiclassical model

\section{Introduction}

Structured light is a matured applied field of study. It has been steadily inventing many new tools and techniques to manipulate and study, from nanoparticles to molecules to atoms. Other chapters of this book have described these developments. 
The purpose of this chapter is to promote the development of out-of-box enquiring questions in physics leveraging the topic of structured light. It is a complex thinking and analytical process to describe a physical phenomenon simply based upon reproducible experimental data. This is because experimental data generation requires detector and deductee to undergo some physical transformation in their relevant parameters after exchanging some energy guided by some allowed force of interaction between them. Since we cannot directly observe the details of the physical interaction process, we cannot be certain from the properties of the measured data as to which property belongs to the detector and which belongs to the deductee. We have not been addressing this important enquiring question explicitly in physics. The field of structured light is a good test optical phenomenon to explore this enquiring question.

Beams of structured light are generated by using classical optical components and the analytical tools of classical optics, which are Maxwell's wave equation and Huygens-Fresnel diffraction integral (or its advanced versions). Then, the concept of "indivisible light quanta" must have come from Einstein's paper on photoelectric effect [1]. However, Lamb et al. [2-4] have clearly shown that the use of semiclassical model, classical light, and quantized atoms yields a much more causal and selfconsistent model for light-matter interactions. This chapter, therefore, strengthens this concept behind the semiclassical model by underscoring some neglected but fundamental nature of light from two fundamental angles- "We never see light" (Section 2) and "Light does not see light" (Section 3). Then we discuss the consequences of assigning detector's intrinsic quantum properties to the energy donating classical and Maxwellian light waves as we do for the photoelectric effect (Section 4). Next, in Section 5, we discuss the consequences of ignoring interaction process visualization, which guides us to accept the necessity of introducing the concept of hybrid photon model. Hybrid photon model eliminates the need for accepting the postulate of "wave-particle duality." This duality postulate actually originated in late 1600s during debate between Newton ("corpuscular") and Huygens ("secondary wavelets"), and they agreed that their debate arose out of their ignorance about the deeper structure of light. Unfortunately, founders of mathematical formalism of quantum have promoted the "wave-particle duality" as the new confirmed knowledge. In reality, this postulate should energize us to keep exploring the deeper issues behind quantized emission and absorption of light and classical propagation properties of light. The last section on discussion underscores that we should always try to reevaluate working theories beyond its prevailing successes so we can advance our current understanding. Then discover new phenomena and then invent new tools and technologies.

\section{We never "see" light}

We only perceive or measure the physical transformation induced by light energy in material bodies, which have their own unique response characteristics due to their unique response properties to light. Therefore, assigning any new physical property, to a physical entity under study, should be done carefully to ascertain that the observed (measured) property is not that of the detecting entity. This is especially important for light. We always infer the incidence (presence) of light after observing (or perceiving) some physical transformation in the detecting element. It could take place through a wide variety of already known phenomena like photoelectric effect, photochemical effect, photothermal effect, photo-acoustic effect, etc. In all such cases, a finite amount of energy from the EM wave is absorbed by the detecting element to undergo some quantitative physical 
transformation, which becomes our data. If the detecting element is inherently quantum mechanical in nature, then the amount of energy $\Delta E_{m n}=h \nu_{m n}$ is absorbed by the detector out of the EM waves and will correspond to the specific quantum transition. However, all light-matter interactions are frequency dependent since all materials are individual dipolar atoms and molecules or their assemblies in solid or liquid states. X-rays and $\gamma$-rays do not interact with quantum mechanical Siphotoelectric detectors or classical photothermal detectors. We need appropriate materials where X-rays can stimulate the electrons in the inner shells of atoms and $\gamma$-rays can stimulate the nuclear energy levels at the core of atoms.

The strength of the evidence-based science lies with the corroboration of evidences with a suitable mathematical model. The model must help us to visualize the interaction processes that give rise to measurable data (evidence). This is the foundation of our causal approach to explore the laws of nature. This causal approach allows us to keep refining both the measurements and the modeling as we keep integrating diverse observations into a broader and well-validated theory. This is how our scientific advances have been continuing for centuries. Therefore, let us explore the physical process steps behind the generic detection/interaction processes (Ch. 12, in [5]):

1. All measured data are some quantitative physical transformations in some detector element induced by a deductee-element.

2. All physical transformations must be triggered by some physical interaction (stimulation) process, followed by energy exchange between the detector and the deductee. Discouraging the visualization of such invisible interaction processes has been the key mystifying reason behind our "working" theories, whose purpose has been limited to validate only the measurable data.

3. All energy exchange must be guided (allowed) by some specific and allowed force of interaction existing between the detector and the deductee. Our continuing failure to understand the origin of all forces and unify them is the key bottlenecks behind the causal advancement in modern physics.

4. All forces, short or long range, have finite physical ranges. Therefore, all interactions are fundamentally "local" or physical range dependent.

Thus, we cannot generate observable (measurable) data without some physical transformation in an interactant (detector) whose intrinsic physical properties dictate its specific response characteristics to one or the other force to participate in any interaction, leading to a specified amount of energy exchange leading to the observable transformation. Obviously, recordable data generation is not possible if the interactants are physically beyond the range of their mutual force of interaction. Causal physics require that the interactants recognize each other through their mutually allowed force of interaction. Without a direct hit of a well-collimated laser beam within the active area of a detector of a power meter, we cannot ascertain the energy of the laser beam. Interaction-free data generation cannot take place in the causal world. "Spooky action at a distance" is an unfortunate cultural phenomenon that wants to mystify physics. Nature is systematically causal. That is why our "cause-effect" inter-relating causal mathematical equations, through centuries, have remained the key guiding tool to explore nature. Nature is not mystical.

Measured and analyzed "elliptical polarization" does not imply that the resultant electrical vector of the light beam is rotating circularly as the composite light beam (two collinear, phase-steady, and orthogonally polarized beams with $90^{\circ}$ relative 
phase delay) is propagating with the resultant E-vector helically rotating around the Poynting vector. In this assumed and imaginary model, the energy of the composite light beam would have been also oscillating due to time-varying resultant amplitude of the E-vector. This would have implied that nature is violating the law of conservation of energy. Fortunately, in this case, our mathematics has been guiding us along the correct and causal path. Jones' matrix has been constructed to find the final energy of a composite light beam as the sum of the two separate energies contained in each of the two orthogonal polarization. The energy in each of the two orthogonal components is the square modulus of the sum of the $\mathrm{X}$-component amplitudes and the Y-component amplitudes, carried out separately. Interested readers should consult Ch. 9 on polarization phenomenon in Ref. [5]. The chapter underscores, using elementary mathematics and the bulk dipolar polarizability $\chi$, that without explicitly inserting this light-matter interaction parameter, the understanding of the ongoing physical process becomes difficult and confusing.

\section{Light does not "see" light}

The light wave amplitudes cross propagate and co-propagate through each other in the absence of interacting materials. This is why experimental astrophysics can image and analyze individual distant galaxies or stars even though the light selected by a telescope has crossed through the light beams of innumerable galaxies and/or stars. This is the same physical reason why we can see (recognize) each other from a distance, even though the scattered light beams from innumerable other faces and objects are crossing through each other. Alhazen experimentally validated this noninteraction of waves, or NIW, about a thousand years ago [6]. This brilliantly simple experiment is sketched in Figure 1.

Alhazen generated the inverted images of a set of candles through a pinhole camera. He found that blocking anyone or more candles does not create any changes in the images of the other candles. Inverted images clearly underscored that the light from all the candles were crossing through each other at the tiny pinhole. Alhazen underscored that he did not understand the deeper nature of light. He was humble.

Much later, Huygens formally postulated NIW in his 1690 book [7] when he presented his principle of wave propagation visualizing the process as the perpetual generation of innumerable secondary wavelets out of every point on the wave front. This also implied that the space is an energetic tension field to be able to support the perpetual wavelet generation and propagation (Ch. 11 in [5, 8-10]). Huygens explicitly articulated non-interaction of waves (Figure 2):

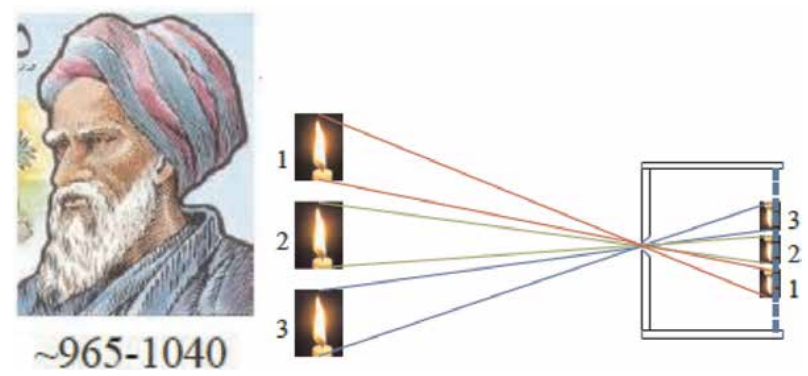

Figure 1.

Alhazen's ancient experiment forced him to conclude that light does not interact with light. We still are struggling with the wave-particle duality [5]. 


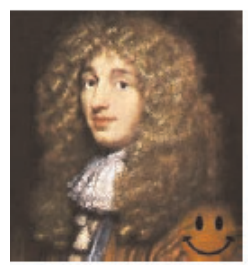

$1629-1695$

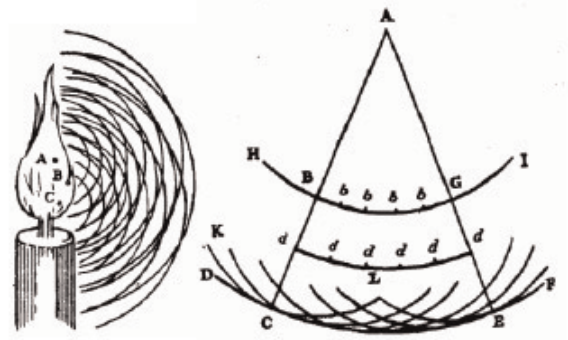

The

geometry is

based on the

assumption

of the NIW-

property.

Figure 2.

Huygens' visualization of the wave propagation process through indefinite number of secondary wavelets, diffractively evolving through each other, "unperturbed by each other, cross one another without hindering one another in any way" [7]. This is non-interaction of waves (NIW) [5].

For I do not find that any one has yet given a probable explanation of the first and most notable phenomena of light, namely ......how visible rays, coming from an infinitude of diverse places, cross one another without hindering one another in any way. From p. 2 in [7].

In p. 4 of his 1690 book, Huygens clearly anticipated the existence of a universal tension field, like pressure tension of air, but without any material particles, which facilitates the perpetual propagation of waves, as sound does in the air.

In 1817, Fresnel gave the mathematical structure to the Huygens principle in which NIW was automatically built in Huygens-Fresnel diffraction integral (HFDI). The integral literally propagates innumerable spherical wavelets that keep evolving through each other while diffractively evolving as co-propagating and cross propagating through each other $[11] . E\left(P_{\text {det. }}\right)$ is the resultant amplitude:

$$
E\left(P_{\text {det. }}\right)=\frac{-i}{\lambda} \iint_{\Sigma} U\left(P_{\text {source. }}\right) \frac{\exp \left(i k r_{s \rightarrow d}\right)}{r_{s \rightarrow d}} \cos \theta d s
$$

Notice that the above HFDI propagates all spherical wave fronts out of every source point to the detector plane, irrespective of its distance $r_{s \rightarrow d}$ from the source plane. These amplitude wave fronts evolve through each other completely independent of each other irrespective of how far they are propagating. In other words, the HFDI has automatically incorporated the NIW property of the waves.

After Maxwell's wave equation was developed (1867), it was found that HFDI is a solution to Helmholtz equation, a time-independent form of Maxwell's generalized wave equation. Maxwell's wave equation accepts any linear superposition of wave amplitudes as its solution. The physical meaning, in the context of NIW, is that wave properties of the individual propagating wave remain unaltered as they cross propagate and/or co-propagate through each other. In other words, light does not interfere with light in the absence of interacting material, which we have logically derived in section on "We do not see light," except through the "eyes" of interacting materials.

It is then obvious that the generation and spatial superposition of multiple complex light wave fronts will continue to diffractively evolve and co-propagate as independent beams. However, when they finally interact with some materials, the energy transfer to any interacting material will be the square modulus of the sum of the finally evolved "local" wave front incident on the material. If the material is an anisotropic polarized detector, it will respond to the square modulus of the sum of all the amplitude components projected on to its polarization axis. If it is a very small-suspended anisotropic particle and the state of polarization is rotating slowly 
in time and if the inertia permits, the anisotropic particle will rotate with the rotating polarizing field since most materials strongly respond to the resultant Evector. However, the original set of multiple co-propagating wave amplitudes does not reorganize themselves into a single composite wave front because of the overriding NIW property of waves.

\section{Consequences of assigning detector's properties to the energy donating entity}

Can we logically confirm that the emission of a single photoelectron proves the existence of light as "indivisible light quanta"? We should recognize that the individual "clicks," which we register in photon counting electronics, are actually a brief current pulse, probably, consisting of billions of amplified electrons. It may not be difficult to validate that this highly amplified current pulse has been originally triggered by a single photoelectron. However, releasing a quantummechanically bound electron does not necessarily require the presence of a quantum photon of energy $h \nu_{m n}=\Delta E_{m n}$. The quantum cupful of energy $\Delta E_{m n}$ can be acquired by a quantum entity from almost any source of energy under appropriate condition of interactions. Ancient humans used to create fire by using sparks generated by fast mechanical collisions between a pair of stones. They had no idea that they were inducing quantum transitions in the molecules of the stones while transferring the classical kinetic energy out of their moving hands! This is why the quantum formalism does not require any quantum postulate that energy providers to induce quantum transitions have to have energy-matching quantum states. In fact, Boltzmann's classical statistical thermal population density formula has been co-opted by the quantum mechanics. Un-quantized thermal energy can be absorbed during thermal collisions by quantum entities to fill up their quantum cups while accepting only that much of energy that fills up their quantum cups [12].

Toward the end of his life, Einstein, the originator of the concept of "indivisible light quanta," clearly stated that even after "50 years of brooding," he still did not understand "what are light quanta" [13]. Author was inspired by Einstein's doubt and delved into exploring the nature of light for many decades [14-16].

In the section on "We do not see light," we have underscored that detectors see light based on their internal physical properties. This is why visual observation of classical interferometry never pointed us to the quantumness of light. In fact, Newton was the first inventor of two-beam interferometry. He measured the radius of curvature of his plano-convex lens by putting it on a flat mirror and shining light from the top. Note that the debate over wave-particle duality started long time ago during late 1600 by Newton ("corpuscular") and Huygens ("wavelet"). Nevertheless, they recognized that their debate represented their deeper ignorance about the fundamental nature of light. We still have not fully understood the deeper nature of light. Therefore, we should not make the "wave-particle duality" as our confirmed new knowledge. We should humbly continue to explore the deeper nature of light. That is the purpose of this chapter.

The first solid reasoning behind theorizing emission and absorption of light from materials in discrete energy packets was presented by Planck in 1900 to match analytically the already measured blackbody radiation curve. However, Planck maintained his understanding that the quantum processes are real only during the instants of emission and absorption. After emission, the EM energy packet immediately evolves into Huygens' diffractive wavelets. This is how the diffractive radiation achieves the state of equilibrium energy density within the enclosed blackbody cavity [14]. 


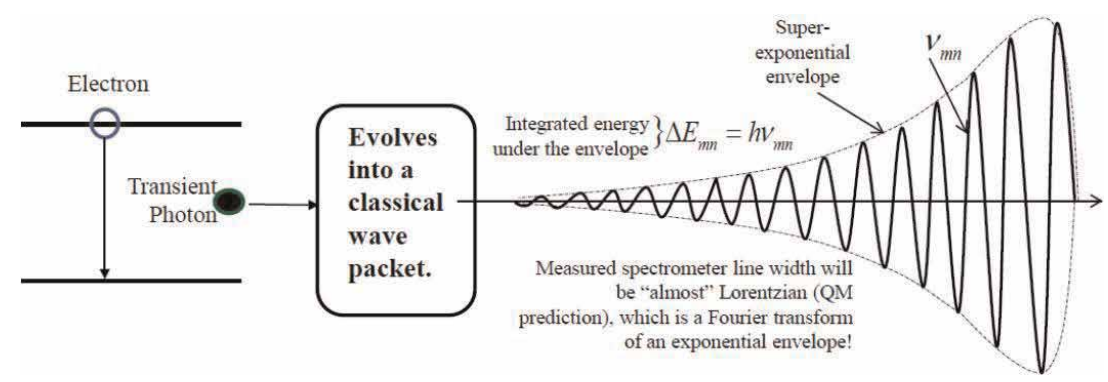

Figure 3.

Transient quantum photon, immediately after emission from an atom, evolves into a diffractively spreading classical wave packet with quasi-exponential temporal envelope. All the quantum predictions are preserved. The total energy carried by the wave packet is $\Delta E_{m n}=h \nu_{m n}$ with the unique carrier frequency $\nu_{m n}$. The Fourier transform of the quasi-exponential envelope gives a spectral line width close to Lorentzian, which is the normal spectral line width of spontaneous emissions.

However, during very late 1800 , emission of photoelectrons from photocathode showed some uniqueness. Below some optical frequency, there was no photoelectron emission in spite of increasing the radiation density. Young Einstein correctly surmised that there is some "quantumness" hidden behind this frequency dependence and no electron emission after a cutoff frequency. Unfortunately, Einstein assigned this quantumness to light, instead of to the electrons. However, we now know that electrons are always bound quantum mechanically in all materials, even when they are bound collectively in quantum energy bands in solid state [15]. Here, we must recognize that Bohr atom was formulated in 1913 and Quantum Mechanics was formulated in 1925, many years after the 1905 paper by Einstein on the photoelectric effect. However, had Einstein correctly assigned the quantumness, observed in photoelectric effect, to electrons instead of to light, he would have been able to formulate quantum mechanics the Einsteinian way some 20 years earlier.

We believe that Planck's view of light is correct. EM waves are classical waves, solutions of Maxwell's equation, and propagates following Huygens secondary wavelets. In fact, Refs. [2-4] have derived the equation for photoelectric emission using the semiclassical method. Here, we present a heuristic approach to present the hybrid photon model that is a quantum at the moment of emission but a classical wave packet with a quasi-exponential temporal envelope. The total energy under the envelope corresponds to the QM predictions: (i) total energy of the wave packet is $\Delta E_{m n}=h \nu_{m n}$, and (ii) the quasi-exponential envelope assures the observed spontaneous emission line width as very close to Lorentzian, the Fourier transform of the amplitude envelope of the light pulse (see Figure 3).

\section{Consequences of ignoring interaction process visualization: the necessity of the hybrid photon model}

Let us first recognize that Einstein's photoelectric equation is an energybalancing equation to match the observed data. This is measurable data modeling epistemology (MDM-E). We need to incorporate interaction process mapping epistemology (IPM-E) over and above MDM-E. Einstein's formulation did not embody light-matter interaction process, as we have underscored in the section on "Light does not see light." For accurate semiclassical derivation of the photoelectric effect, the readers should consult the following references [2-4]. We will present here only a heuristic derivation of Einstein's energy-balancing photoelectric equation, but starting from light-matter amplitude-amplitude stimulation, the E-vector of light 

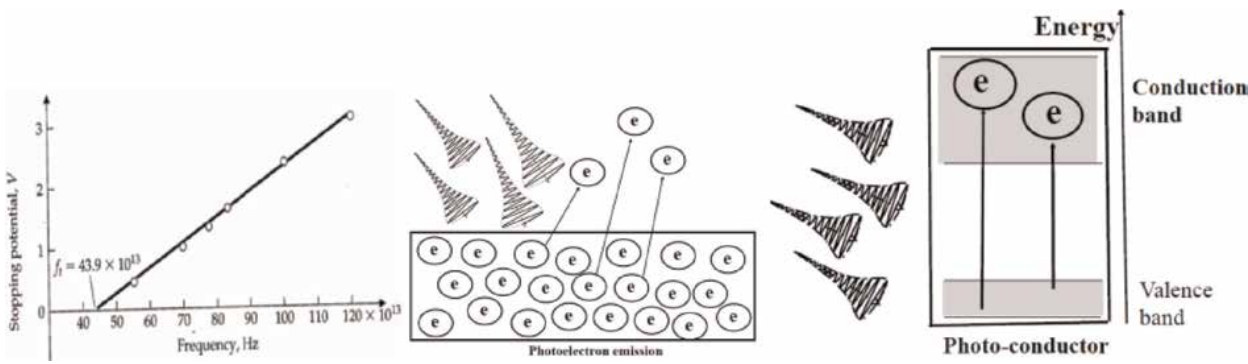

Figure 4.

Left diagram: Emission of photoelectrons from a given material stops at a fixed specific frequency [16]. Middle diagram: Photoelectron emission from photocathode. Right diagram: Photoelectron transfer from valence to conduction band.

stimulating the dipoles as $\chi\left(\nu_{q}\right) E\left(\nu_{q}\right)$, containing the bound electrons, where $\chi\left(\nu_{q}\right)$ is the polarizability, or the light-matter interaction parameter. Since semiclassical thermal radiation consists of random wave groups emitted spontaneously with random phases, the total dipolar amplitude-amplitude stimulation of a bound electron can be expressed as

$$
\Psi=\sum_{q} \psi_{q}=\sum_{q} \chi\left(\nu_{q}\right) E\left(\nu_{q}\right)
$$

The bound electron system must absorb the necessary amount of quantum-cupfilling energy, before the electron can be released to the conduction band or become a free-space electron. This energy exchange is a quadratic process:

$$
|\Psi|^{2}=\left|\sum_{q} \chi\left(\nu_{q}\right) E\left(\nu_{q}\right)\right|^{2} \propto h \nu_{q}
$$

For any quantum system, we must take the ensemble average. A single event (data point), as in Eq. (3), is never sufficient to verify a theory:

$$
\left\langle|\Psi|^{2}\right\rangle=\left\langle\left|\sum_{q} \chi\left(\nu_{q}\right) E\left(\nu_{q}\right)\right|^{2}\right\rangle \Leftrightarrow\left\langle h \nu_{q}\right\rangle=\left\langle\phi_{\text {work fn. }}+(1 / 2) m \mathrm{v}_{\text {el. }}^{2}\right\rangle
$$

In the right segment of Eq. (4), we have "recovered" Einstein's photoelectric energy-balancing equation out of dipole amplitude stimulations due to multitudes of waves. The left curve in Figure 4 represents the photoelectric current [15]. Waves only fill up the quantum cups with the necessary energy if the dipoles are resonant to the frequency $\nu$ of the incident waves.

\section{Discussions}

Visible light is always generated through orbital quantum electron transition processes in atoms. We have presented our hybrid photon model where light is released as a quantum energy packet, $h \nu$, as required by quantum formalism. Then we posit that immediately after the release of the $h \nu$ packet, it evolves into a classical wave packet and follows Maxwell's wave equation and Huygens-Fresnel diffraction integral. We generate structured light using classical optical components and classical optical analytical tools. The possibility of introducing any quantumness in classical light during this process is difficult to imagine. The author is suggesting that we should explore the physical response properties of the material particles that 
are inserted into the structured beams and try to visualize the light-matter dipolar interaction processes. This would be a better system-engineering approach to understand different optical phenomena $[17,18]$.

The key point of the author is that our unquestioned acceptance of the waveparticle duality has been hindering our deeper enquiry into the ultimate nature of light. The author has been attempting to inspire this process over decades by organizing special publications [13] and special conference series at SPIE from 2005 to 2015 [19], publishing experimental papers [20], and writing books [5, 21]. During this long arduous process, the author has recognized that Huygens principle (HP) of "secondary wavelets" has deeper enduring value for physics. HP requires space to be a physical tension field, a complex tension filed or CTF, to facilitate the perpetual and well-defined velocity of light. CTF possesses the necessary characteristic properties, which facilitates the perpetual velocity of light, $c=\left(\varepsilon_{0}^{-1} / \mu_{0}\right)^{1 / 2}$, through the entire cosmic space. CTF must also possess other physical attributes that we have not been exploring actively. Thus, CTF could have serious implications in guiding us to reorganize our investigations to fulfill Einstein's dream of defining a unified field. CTF could be behind the emergence of both the EM waves and the particles as different kinds of oscillations of the same CTF, which holds $100 \%$ of the energy of our cosmic system (Ch. 11 in $[5,8,17])$.

Optical physicists should note that the two major "successful theories" for gravity, those of Newton and of Einstein, have been unable to explain the velocity distribution curves measured for a couple hundred galaxies. Therefore, theoretical physicists have proposed unnecessary postulates of the existence of Dark Matter and Dark Energy, neither of whose existence has been confirmed over the last
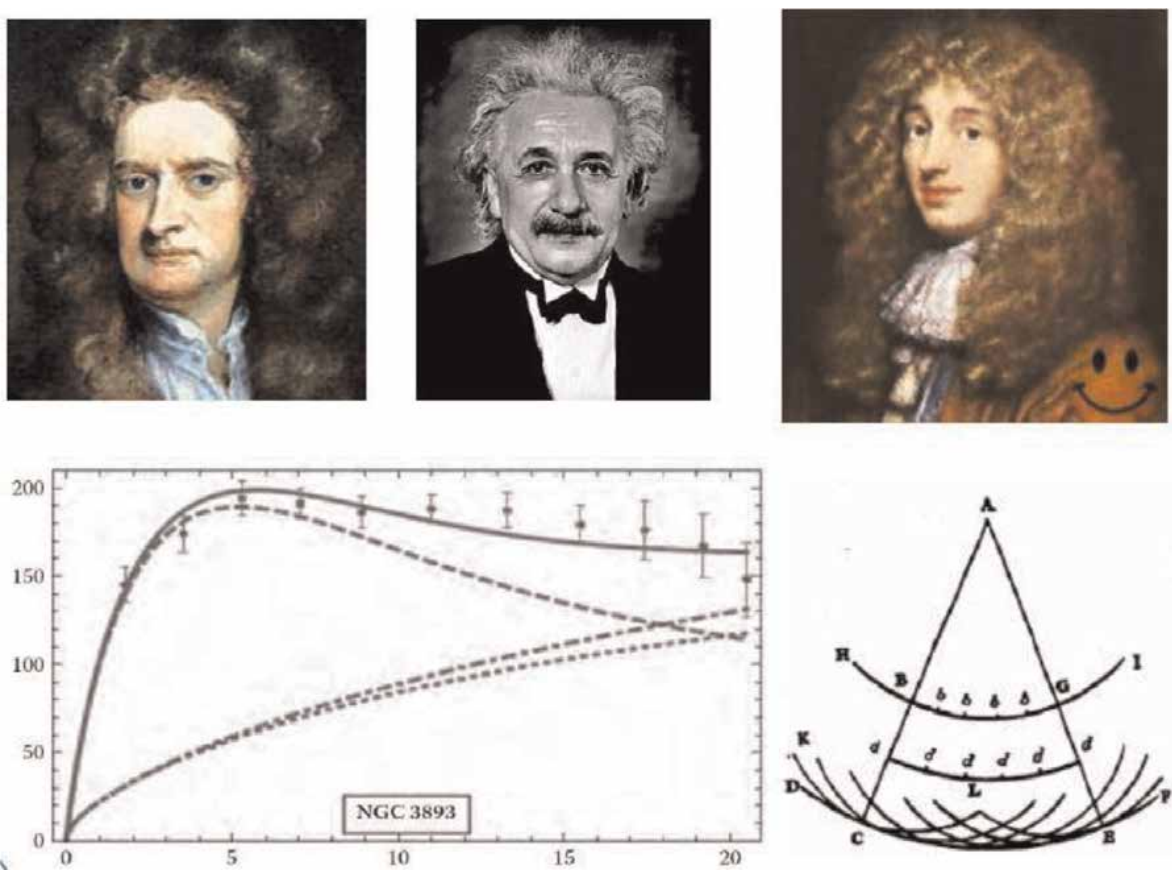

Figure 5.

Left-bottom curves [22]: Gravity theories of Newton and Einstein cannot match the measured velocity distribution of stars in one particular galaxy. This phenomenon turns out to be true for a couple of hundreds of galaxies. These two theories are very accurate for our solar system, but not good at the galactic scale. Bottom right: Huygens 1690 postulate of secondary wavelets [7], framed into Huygens-Fresnel diffraction integral, is still the core guiding analytical equation for the broad field of physical optics, including generating structured light. 
several decades. These theories are definitely not wrong. Those who have been successfully launching and manipulating artificial satellites in our solar system rarely need to go beyond the mechanics of Newtonian gravity. Einstein's gravity correctly predicts the precession of the perihelion of Mercury. However, these two theories must be limited in capability to model gravity in the galactic scale (Figure 5).

In contrast, in spite of subtle mathematical issues behind the Huygens postulate [23] of secondary wavelets, it remains the key foundational guide to propagate light through free space and non-interacting materials. To model light-matter interaction, Maxwell's equations have remained quite successful. In the history of physics, all theories eventually yield to new and better theories. Our attempts should be directed along these lines. We should not try to keep promoting the general validity of all working theories.

Optical physicists should explore the deeper enduring values behind the Huygens principle and find the limits of its application in different optical phenomena to advance further optical physics. Studies in optical phenomena have been guiding major advances in physics since ancient times. Starting from the 1600 s to 1800 s, advancements in physics were predominantly pioneered by scientists studying the broad field of optical sciences. However, starting from the early 1900s, this pioneering role has been shifted from guiding fundamental physics to finding only novel technical applications of optics. It is time for optical physicists to pick up more proactive roles in guiding the development of fundamental physics [24].

\section{Author details}

ChandraSekhar Roychoudhuri

Department of Physics, University of Connecticut, Storrs, CT, USA

*Address all correspondence to: chandra.roychoudhuri@uconn.edu

\section{IntechOpen}

(C) 2019 The Author(s). Licensee IntechOpen. This chapter is distributed under the terms of the Creative Commons Attribution License (http://creativecommons.org/licenses/ by/3.0), which permits unrestricted use, distribution, and reproduction in any medium, provided the original work is properly cited. (cc) BY 


\section{References}

[1] Einstein A. Concerning a heuristic point of view about the creation and transformation of light. Annalen der Physik. 1905;17:132-148

[2] Lamb WE, Scully MO. Polarization, matter and radiation, Jubilee volume in honor of Alfred Kastler. In:

Photoelectric Effect without Photons.

Paris: Presses Universitaires de France; 1969. pp. 363-369

[3] Grynberg G, Aspect A, Fabre C. Introduction to Quantum Optics: From the Semi-Classical Approach to Quantized Light. Cambridge University Press; 2010

[4] Stroud CR, Jaynes ET. Long-term solutions in semi-classical radiation theory. Physical Review A. 1970;1(1): 106-121

[5] Roychoudhuri C. Causal Physics: Photon by Non-Interaction of Waves. Boca Raton: Taylor \& Francis; 2014

[6] Ronchi V. Nature of Light. Cambridge: Harvard University Press; 1970. p. 53

[7] Huygens C. Treatise on Light, drafted in 1678. English translation. Chicago: University of Chicago Press; 2005 Project Gutenberg Edition: Available from: http://www.gutenberg. org/ebooks/14725

[8] Roychoudhuri C. Next frontier in physics-Space as a complex tension field. Journal of Modern Physics. 2012

[9] Roychoudhuri C. Could space be considered as the inertial rest frame? In: Proc. SPIE. Vol. 9570-30; 2015

[10] Roychoudhuri C. The concept of "fundamental" must keep evolving, FQXi essay competition. 2015. Available from: https://fqxi.org/data/essay-contest-files/ Roychoudhuri_180121_Fundame.pdf
[11] Born M, Wolf E. Principles of Optics. 6th ed. Cambridge University Press; 1980

[12] Roychoudhuri C. Hybrid photon model bridges classical and quantum optics.In: JW3A.32-1, OSA Annual Conference; 2017

[13] Roychoudhuri C, Roy R, editors. The nature of light. What is a photon? Optics \& Photonics News (OSA). 2003. Available from: https://www.osa-opn. org/opn/media/Images/PDFs/3185_ 6042_30252.pdf?ext=.pdf

[14] Planck M. The Theory of Heat Radiation. Blakistons Son \& Co.; 1914. Free download from Gutenberg eBooks

[15] Hufner S. Photoelectron Spectroscopy: Principles and Applications. Springer; 2010

[16] Bernstein J, Fishbane PM, Gasiorowicz S. Modern Physics. Prentice Hall; 2000

[17] Roychoudhuri C. Urgency of evolution process congruent thinking in physics. In: An advocacy to elevate the prevailing abstract physics-Thinking towards a functionally useful reverseengineering thinking, Proc. SPIE. Vol. 9570; 2015

[18] Roychoudhuri C. Consequences of repeated discovery and benign neglect of non-interaction of waves (NIW). In: Proc. SPIE. Vol. 10452 1045215; 2017. Available from: https://spie.org/Publications/ Proceedings/Paper/10.1117/12.2266216

[19] Roychoudhuri C. et al. Key organizer of the conference series: Nature of light: What (is) are photons? In: Proc. SPIE Vol. 5866, 2005; Proc. SPIE Vol. 6664, 2007; Proc. SPIE Vol. 7421, 2009; Proc. SPIE Vol. 8121, 2011; Proc. SPIE Vol. 8832, 2013; Proc. SPIE Vol. 9570, 2015 
[20] Roychoudhuri C. Most of the author's papers and presentations for workshop, etc. Available from: http:// www.natureoflight.org/CP/

[21] Roychoudhuri C, Kracklauer AF, Creath K. The Nature of Light: What Is a Photon? Boca Raton: Taylor \& Francis; 2008

[22] Mannheim PD, O’Brian JG. Fitting galactic rotation curves with conformal gravity and a global quadratic potential. Physical Review D. 2012;85:124020

[23] Goodman J. Fourier Optics. W. H. Freeman; 2017

[24] Roychoudhuri C, Tirfessa N. Bringing reality in physics: System engineering approach to optical phenomena following Huygens' Principle. In: Proc. SPIE 11143; 2019. DOI: $10.1117 / 12.2523602$. Available from: https://www.spiedigitallibrary. org/conference-proceedings-of-spie on 08 Jul 2019 


\title{
Chapter 2
}

\section{Quantized Field of Single Photons}

\author{
Constantin Meis
}

\begin{abstract}
We present theoretical developments expressing the physical characteristics of a single photon in conformity with the experimental evidence. The quantization of the electromagnetic field vector potential amplitude is enhanced to a free of cavity photon state. Coupling the Schrödinger equation with the relativistic massless particle Hamiltonian to a symmetrical vector potential relation, we establish the vector potential - energy equation for the photon expressing the simultaneous waveparticle nature of a single photon, an intrinsic physical property. It is shown that the vector potential can be naturally considered as a real wave function for the photon entailing a coherent localization probability. We deduce directly the electric and magnetic field amplitudes of the cavity-free single photon, which are revealed to be proportional to the square of the angular frequency. The zero-energy electromagnetic field ground state (EFGS), a quantum vacuum real component, issues naturally from Maxwell's equations and the vector potential quantization procedure. The relation of the quantized amplitude of the photon vector potential to the electronpositron charge results directly showing the physical relationship between photons and electrons-positrons that might be at the origin of their mutual transformations. It becomes obvious that photons, as well as electrons-positrons, are issued from the same quantum vacuum field.
\end{abstract}

Keywords: single photon, vector potential, photon wave-particle equation, photon wave function, photon electric field, electromagnetic field ground state, electron-positron charge

\section{Introduction}

During the last decades, an impressive technological development has been achieved permitting the manipulation of single photons with a high degree of statistical accuracy. However, despite the significant experimental advances, we still do not have a clear physical picture of a single photon state universally accepted by the scientific community, especially involved in quantum electrodynamics. In this chapter, based on the present state of knowledge, we make a synthesis of the physical characteristics of a single photon put in evidence by the experiments, and we advance theoretical developments for its representation. Accordingly, the concept of the wave-particle nature of a single photon becomes physically comprehensive and in agreement with the experimental evidence.

However, before advancing in the theoretical developments, we consider that it is important starting with a brief historical review on the efforts carried out previously for understanding the nature of light while simultaneously making a synthesis of the main experimental results which are of crucial importance for the comprehension of the birth of the photon concept. 
The very first scientific publications on the nature of light are due to ancient Greeks who believed light is composed of corpuscles [1, 2]. Around 300 BC Euclid published the book Optica in which he developed the laws of reflection based on the rectilinear propagation of light. Two centuries later, Ptolemy of Alexandria published the book Optics, in which he included extensively all the previous knowledge on light. In this book, colours as well as refraction of the moonlight and sunlight by the earth's atmosphere were analysed. After Ptolemy of Alexandria, almost no progress has been reported until the seventeenth century.

In the year of 1670, Newton revived the ideas of ancient Greeks and advanced the theory following that light is composed of corpuscles that travel rectilinearly [3]. Ten years later, Huygens developed the principles of the wave theory of light $[1,4,5]$. Huygens' wave theory was a hard opponent to Newton's corpuscle concept. In the beginning of the nineteenth century, Young obtained experimentally interference patterns using different sources of light and explained some polarisation observations by assuming that light oscillations are perpendicular to the propagation axis $[1,6]$. Euler and Fresnel explained the diffraction patterns observed experimentally by applying the wave theory [6]. In 1865, Maxwell published his theory on the electromagnetic waves establishing the relations between the electric and magnetic fields and showing that light is composed of electromagnetic waves [7]. A few years later, Hertz confirmed Maxwell's theory by discovering the longwavelength electromagnetic radiation [1,7]. Thus, at the end of the nineteenth century, the scientific community started to accept officially the wave nature of light replacing Newton's theory.

Nevertheless, new events supporting the particle nature of light occurred in the beginning of the twentieth century. Stefan and Wien discovered the direct relationship between the thermal radiation energy and the temperature of a black body $[8,9]$. However, the emitted radiation energy density as a function of the temperature calculated by Rayleigh failed to describe the experimental results at short wavelengths. Scientists had given the name "UV catastrophe" to this problem revealing the necessity of a new theoretical approach. Planck managed to establish the correct energy density expression for the radiation emitted by a black body with respect to temperature, in excellent agreement with the experiment [8]. For that purpose, he assumed that the bodies are composed of "oscillators" which have the particularity of emitting the electromagnetic energy in "packets" of $h \nu$, where $\nu$ is the frequency and $h$ is a constant that was later called Planck's constant. During the same period, the experiments carried out by Michelson et al. [10] demonstrated that the speed of light in vacuum is a universal physical constant corresponding to the product of the frequency $\nu$ times the wavelength $\lambda$, that is, $c=\lambda \nu$.

In 1902, Lenard pointed out that the photoelectric effect, discovered by Hertz 15 years earlier [11], occurs beyond a threshold frequency of light and the kinetic energy of the emitted electrons does not depend on the incident light intensity. Based on Planck's works, Einstein proposed a simple interpretation of the photoelectric effect assuming that the electromagnetic radiation is composed of quanta with energy $h \nu$ [12]. He advanced that the energy of a light ray when spreading from a point consists of a finite number of energy quanta localised in points in space, which move without dividing and are only absorbed and emitted as a whole. Although that was a decisive step towards the particle theory of light, the concept of the light quanta was still not generally accepted, and Bohr, who was strongly opposed to the particle concept of light [13], announced in his Nobel lecture (1922) that the light quantum hypothesis is not compatible with the interference phenomena and consequently it cannot throw light in the nature of radiation. Bohr's statement was rather surprising because Taylor's experiments, consisting of repeating Young's double slit diffraction at extremely low light intensities, had already demonstrated since 1909 that light rays 
are composed of discrete parts whose spots compose the diffraction patterns by gradual accumulation on the detection screen [14]. Compton published his studies on X-rays scattered by free electrons in 1923 advancing that the experimental results could only be interpreted based on the light quanta model [15].

Thus, the photoelectric effect and Compton scattering have been initially considered as the undoubtable demonstrations of the particle nature of light and historically were the strongest arguments in favour of the light quanta concept, which started to be universally accepted, and Lewis introduced the word "photon", from the Greek word phos ( $\Phi \omega$ s, which means light) [1, 4].

Therein, it is extremely important to mention that Wentzel in 1926 [16] and Beck in 1927 [17], as well as much later Lamb and Scully in the 1960s [18], demonstrated that the photoelectric effect can be interpreted remarkably well by only considering the wave nature of light, without referring to photons at all [19]. Furthermore, the Compton scattering has been fully interpreted by Klein and Nishina in 1929 [20] also by considering the electromagnetic wave nature of light without invoking the photon concept. On the other hand, Young's experiment, initially presented as the most convincing argument for the wave nature of light, was applied by Taylor at very low intensities to demonstrate the particle concept of light [14]. Indeed, much later Jin et al. [21] published an excellent theoretical interpretation of Young's diffraction experiments based only on the particle representation of light.

Thus, the picture on the nature of light in the 1930s was rather confusing since both opposite sides defending the wave or the particle nature advanced equally strong arguments. Hence, Bohr, inspired by de Broglie's thesis on the simultaneous wave character of particles, announced the complementarity principle according to which light has both wave and particle natures appearing mutually exclusively in each specific experimental condition $[1,2,19]$.

The development of lasers [22] in the 1960s and the revolutionary parametric down-convertion techniques $[23,24]$ in the 1970 s, have made it possible to realise conditions in which, with a convenient statistical confidence, only a single photon may be present in the experimental apparatus. In this way, the double-prism experiment [25] realised in the 1990s contradicted for the first time Bohr's mutual exclusiveness demonstrating that a single photon exhibits both the wave and particle natures in the same experimental conditions.

According to the experimental investigations, it has been always stated that a photon has circular, left or right, polarisation with spin $\pm h / 2 \pi$ and cannot be conceived along the propagation axis within a length shorter than its wavelength [26]. Indeed, since Mandel's experiments in the 1960s [27, 28], all the efforts to localise precisely a single photon remained fruitless yielding the conclusion is that a photon cannot be better localised than within a volume of the order of the cube of its wavelength [29, 30]. Furthermore, Grangier et al. demonstrated experimentally the indivisibility of photons $[19,31]$, while in recent years the entangled state experiments $[29,30]$ have shown that the photon should be locally an integral entity during the detection procedure but with a real non-local wave function.

The lateral expansion of a single photon, considered locally as an indivisible entity, was always an intriguing part of physics. With the purpose of studying the lateral expansion of the electromagnetic rays, Robinson in 1953 [32] and Hadlock in 1958 [33] carried out experiments using microwaves crossing small apertures and deduced that no energy is transmitted through apertures whose dimensions are smaller than roughly $\sim \lambda / 4$. In 1986, for the same purpose, Hunter and Wadlinger $[34,35]$ used X-band microwaves with $\lambda=28.5 \mathrm{~mm}$ and measured the transmitted power through rectangular or circular apertures of different dimensions. They concluded that no energy is transmitted when the apertures are 
smaller than $\sim \lambda / \pi$ confirming that the lateral expansion of the photons is a fraction of the wavelength.

Thus, the experiments have shown that the single photon is not a point and cannot be localised at a coordinate, as stated by Einstein, while it can exhibit both the wave and particle natures in the same experimental conditions contradicting Bohr's mutual exclusiveness. However, quantum electrodynamics (QED) has been developed during the 1930s to 1960s based upon the point particle model for the photon [36-39]. In fact, the point photon concept has permitted to establish an efficient mathematical approach for describing states before and after an interaction processes [19,39-41], but it is naturally inappropriate for the description of the real nature of a single photon.

Finally, what we can essentially draw out by summing up the experimental evidence is that a single photon is a minimum, local, indivisible part of the electromagnetic field with precise energy $h \nu$ and momentum $h \nu / c$, having circular left or right polarisation with spin $\pm h / 2 \pi$, respectively. It is not a point particle since it expands over a wavelength $\lambda$ along the propagation axis and is detectable within a volume of the order of $\lambda^{3}$ entailing that its lateral expansion is a fraction of its wavelength. Hence, it appears to be a local "wave-corpuscle" guided by a non-local wave function, absorbed and emitted as a whole and capable of interacting with charges increasing or decreasing its frequency and consequently its energy.

In what follows, we present first the standard theoretical representation of the electromagnetic field quantization resulting in photons, and next we proceed to recent advances based on the vector potential quantization enhanced to a single photon state.

\section{The electromagnetic field vector potential}

\subsection{Reality of the vector potential}

Since the formulation of Maxwell's equations, the vector potential $\vec{A}(\vec{r}, t)$ was considered as a pure mathematical artefact $[4,5,7]$ used to calculate the electric field:

$$
\vec{E}(\vec{r}, t)=-\partial \vec{A}(\vec{r}, t) / \partial t-\vec{\nabla} \Phi(\vec{r}, t)
$$

where $\Phi(\vec{r}, t)$ is the scalar potential, as well as the magnetic field:

$$
\vec{B}(\vec{r}, t)=\vec{\nabla} \times \vec{A}(\vec{r}, t)
$$

In 1949, Ehrenberg and Siday were the first to put in evidence the influence of the vector potential on charged particles [42] deducing that it is a real physical field. Ten years later, Aharonov and Bohm re-infirmed the influence of the vector potential on electrons in complete absence of electric and magnetic fields [43]. That was confirmed experimentally by Chambers [44], Tonomura et al. [45], and Osakabe et al. [46] demonstrating without any doubt the reality of the vector potential field end its direct influence on charges.

From a theoretical point of view [43], the behaviour of a particle with charge $q$ and mass $m$ in the vicinity of a solenoid where the vector potential is present is described by the Hamiltonian: 


$$
H=\frac{1}{2 m}(-i \hbar \vec{\nabla}-q \vec{A}(\vec{r}, t))^{2}+q \Phi(\vec{r}, t)
$$

with $\hbar=h / 2 \pi$ as Planck's reduced constant.

If the solenoid is extremely long along the $z$ axis, then the magnetic field is uniform in the region inside and zero outside. The scalar potential $\Phi$ can be put to zero by assuming that the solenoid is not charged. In this case, in the outside region, the electric and magnetic fields are zero, but the vector potential is not zero and depends on the magnetic field flux in the solenoid:

$$
\vec{A}=\frac{1}{2 \pi r} \int_{s} \vec{B} \cdot d \vec{S} \hat{e}_{\theta}
$$

where $r$ is the radial distance from the $z$ axis of the solenoid, $S$ is the surface of the circle with radius $r$ perpendicular to $z$, and $\hat{e}_{\theta}$ is the angular unit vector in cylindrical coordinates.

The Schrödinger equation for a charged particle outside the solenoid, where the vector potential is not zero, writes in complete absence of any other external potential:

$$
i \hbar \frac{\partial}{\partial t} \Psi(\vec{r}, t)=\frac{1}{2 m}(i \hbar \vec{\nabla}+q \vec{A})^{2} \Psi(\vec{r}, t)
$$
tions:

with $\vec{A}$ given by Eq. (4). The solutions of the last equation are the wave func-

$$
\Psi(\vec{r}, t)=\Psi_{p}(\vec{r}, t) e^{\frac{i q}{h} \int_{0}^{\vec{r}} \vec{A}\left(\vec{r}^{\prime}\right) \cdot d \vec{r}^{\prime}}
$$

where $\Psi_{p}(\vec{r}, t)$ is the solution of Schrödinger's equation in absence of the vector potential:

$$
i \hbar \frac{\partial}{\partial t} \Psi_{p}(\vec{r}, t)=-\frac{\hbar^{2}}{2 m} \vec{\nabla}^{2} \Psi_{p}(\vec{r}, t)
$$

The exponential part of the wave function of Eq. (6) entails that two particles have equal charge and mass moving both outside the solenoid at the same distance from the axis, but the first in the same direction with the vector potential $\vec{A}$ and the second in the opposite direction will suffer a phase difference:

$$
\delta \Theta=\frac{q}{\hbar} \int_{s} \vec{B} \cdot d \vec{S}
$$

Interference patterns for electrons in analogue conditions have been observed experimentally [44-46] demonstrating that the vector potential is a real physical field and interacts directly with charged particles in complete absence of magnetic and electric fields and of any other potential.

\subsection{The radiation vector potential: classical to quantum link}

The vector potential, being a real field, is considered as the fundamental link between the electromagnetic wave theory issued from Maxwell's equations and the 
particle concept in quantum electrodynamics (QED) [19, 36, 39]. We will show analytically how this link is established.

In the classical theory $[5,7]$, the energy density of a mode $k$ of the electromagnetic wave writes:

$$
W_{k}(\vec{r}, t)=\frac{1}{2}\left(\varepsilon_{0}\left|\vec{E}_{k}(\vec{r}, t)\right|^{2}+\frac{1}{\mu_{0}}\left|\vec{B}_{k}(\vec{r}, t)\right|^{2}\right)
$$

where $\varepsilon_{0}$ and $\mu_{0}$ are the electric permittivity and magnetic permeability of the vacuum, respectively, related to the speed of light in vacuum $c$ by $\varepsilon_{0} \mu_{0} c^{2}=1$.

In the case of a monochromatic plane wave with angular frequency $\omega_{k}$, the electric $\overrightarrow{E_{k}}(\vec{r}, t)$ and magnetic $\overrightarrow{B_{k}}(\vec{r}, t)$ fields are proportional to the vector potential amplitude $A_{0 k}\left(\omega_{k}\right)$ :

$$
\begin{gathered}
\overrightarrow{E_{k}}(\vec{r}, t)=-2 \omega_{k} A_{0 k}\left(\omega_{k}\right) \hat{\varepsilon} \sin \left(\vec{k} \cdot \vec{r}-\omega_{k} t\right) \\
\overrightarrow{B_{k}}(\vec{r}, t)=-\frac{1}{c} 2 \omega_{k} A_{0 k}\left(\omega_{k}\right)(\hat{k} \times \hat{\varepsilon}) \sin \left(\vec{k} \cdot \vec{r}-\omega_{k} t\right)
\end{gathered}
$$

where $\hat{\varepsilon}$ is a unit vector perpendicular to the propagation axis, $|\vec{k}|=2 \pi / \lambda_{k}$ is the wave vector along the propagation axis, and $\lambda_{k}$ is the wavelength of the mode $k$.

Introducing Eqs. (10) and (11) in Eq. (9), the energy density now depends on the square of the vector potential amplitude:

$$
W_{k}(\vec{r}, t)=4 \varepsilon_{0} \omega_{k}^{2}\left|A_{0 k}\left(\omega_{k}\right)\right|^{2} \sin ^{2}\left(\vec{k} \cdot \vec{r}-\omega_{k} t\right)
$$

The mean value over a period, thus over a wavelength, is time independent:

$$
W_{k}=2 \varepsilon_{0} \omega_{k}^{2}\left|A_{0 k}\left(\omega_{k}\right)\right|^{2}
$$

Note that the last equation expressing the mean energy density over a period of the mode $k$ of the electromagnetic wave is independent on any external volume yielding that in the classical description, a free of cavity electromagnetic radiation mode expands naturally within a minimum volume. In a given cavity, this volume corresponds roughly to that imposed by the boundary conditions and the cut-off wave vectors $[4,5,7]$.

On the other hand, in the quantum description, the energy density for a number $N\left(\omega_{k}\right)$ of $k$-mode photons with angular frequency $\omega_{k}$ and energy $\hbar \omega_{k}$ in a volume $V$ writes simply:

$$
W_{k}=\frac{N\left(\omega_{k}\right) \hbar \omega_{k}}{V}
$$

In order to link the classical to the quantum description $[4,9,19]$, the classical mean energy density over a period, expressed by Eq. (13), is imposed to be equivalent to the quantum mechanics expression of Eq. (14) for $N\left(\omega_{k}\right)=1$ getting the vector potential amplitude for a single $k$-mode photon:

$$
\left|A_{0 k}\left(\omega_{k}\right)\right|=\sqrt{\frac{\hbar}{2 \varepsilon_{0} \omega_{k} V}}
$$


The last relation is the fundamental link between the classical and quantum theory of light which is used to define in QED the vector potential amplitude operators for a single photon $[19,26,29,36-41]$ :

$$
\tilde{A}_{k \lambda}=\sqrt{\frac{\hbar}{2 \varepsilon_{0} \omega_{k} V}} a_{k \lambda} \quad \tilde{A}_{k \lambda}^{*}=\sqrt{\frac{\hbar}{2 \varepsilon_{0} \omega_{k} V}} a_{k \lambda}^{+}
$$

where $a_{k \lambda}$ and $a_{k \lambda}^{+}$are, respectively, the annihilation and creation non-Hermitian operators for a $k$-mode and $\lambda$-polarisation photon.

Therein, it is worth noting that an external arbitrary volume parameter $V$ appears in the vector potential amplitude of the single photon, expressed by Eq. (15), which is supposed to be an intrinsic physical property. This could entail the unphysical interpretation that a single photon in an infinite cavity has zero vector potential, thus zero electric and magnetic fields and consequently zero energy. This ambiguity, which is scarcely quoted in the literature, is lifted by considering that, in the case of a single photon, the volume $V$ in Eq. (15) is equivalent to that defined by the boundary conditions in a cavity for the single radiation mode $k$.

\section{Electromagnetic field quantization and the photon description}

\subsection{Harmonic oscillator representation of the electromagnetic field}

The energy of the electromagnetic field in a volume $V$ considered as a superposition of different $k$-modes and $\lambda$-polarisations is obtained directly from Eq. (13):

$$
E_{E M}=2 \varepsilon_{0} V \sum_{k, \lambda} \omega_{k}^{2}\left|A_{k \lambda}\left(\omega_{k}\right)\right|^{2}
$$

where the summation over the $\lambda$-polarisations takes only two values corresponding to circular left and right [19, 36-41].

Replacing in Eq. (17) the vector potential amplitude and its conjugate by the relations of the vector potential amplitude operators defined in Eq. (16), we get the "normal ordering" radiation Hamiltonian corresponding to the order $a_{k \lambda}^{+} a_{k \lambda}$ of the creation and annihilation operators:

$$
\tilde{H}_{N O}=\sum_{k, \lambda} a_{k \lambda}^{+} a_{k \lambda} \hbar \omega_{k}
$$

and the "anti-normal ordering" Hamiltonian corresponding to the order $a_{k \lambda} a_{k \lambda}^{+}$

$$
\tilde{H}_{A N O}=\sum_{k, \lambda}\left(a_{k \lambda}^{+} a_{k \lambda}+1\right) \hbar \omega_{k}
$$

where we have used the fundamental commutation relation in quantum electrodynamics:

$$
\left[a_{k \lambda}, a_{k \lambda}^{+}\right]=1
$$


In Dirac's representation the eigenfunctions take the simple expression $\left|n_{k \lambda}\right\rangle$, and the action of the creation and annihilation operators of a single $k$-mode and $\lambda$-polarisation photon writes:

$$
a_{k \lambda}^{+}\left|n_{k \lambda}\right\rangle=\sqrt{n_{k \lambda}+1}\left|n_{k \lambda}+1\right\rangle ; \quad a_{k \lambda}\left|n_{k \lambda}\right\rangle=\sqrt{n_{k \lambda}}\left|n_{k \lambda}-1\right\rangle
$$

The successive action of both operators in the normal order corresponds to the photon number Hermitian operator $\tilde{n}_{k \lambda}=a_{k \lambda}^{+} a_{k \lambda}$ having the eigenvalue $n_{k \lambda}$ representing the number of $k$-mode and $\lambda$-polarisation photons:

$$
a_{k \lambda}^{+} a_{k \lambda}\left|n_{k \lambda}\right\rangle=\tilde{n}_{k \lambda}\left|n_{k \lambda}\right\rangle=n_{k \lambda}\left|n_{k \lambda}\right\rangle
$$

In this representation the normal and anti-normal ordering radiation Hamiltonians write, respectively:

$$
\tilde{H}_{N O}=\sum_{k, \lambda} \tilde{n}_{k \lambda} \hbar \omega_{k} ; \quad \tilde{H}_{A N O}=\sum_{k, \lambda}\left(\tilde{n}_{k \lambda}+1\right) \hbar \omega_{k}
$$

We obtain a harmonic oscillator Hamiltonian for the electromagnetic field by considering the mean value of the normal ordering and anti-normal ordering Hamiltonians:

$$
\tilde{H}_{E M}=\frac{1}{2}\left(\tilde{H}_{N O}+\tilde{H}_{A N O}\right)=\sum_{k, \lambda}\left(\tilde{n}_{k, \lambda}+\frac{1}{2}\right) \hbar \omega_{k}
$$

Thus, in QED the electromagnetic field is considered to be an ensemble of harmonic oscillators each represented by a point particle, the photon, whose eigenfunction is denoted simply by $\left|1_{k \lambda}\right\rangle[19,39,41]$.

Although we have no experimental facts showing the harmonic oscillator nature of a single photon, this representation has been adopted since the 1930s [37].

In a different way, a harmonic oscillator representation for the electromagnetic field can be obtained by the intermediate of the canonical variables of position $Q_{k \lambda}$ and momentum $P_{k \lambda}$. For that purpose we introduce the definitions expressing the vector potential amplitude and its complex conjugate with respect to $Q_{k \lambda}$ and $P_{k \lambda}$ $[19,29,41]$ :

$$
A_{k \lambda}=\frac{\left(\omega_{k} Q_{k \lambda}+i P_{k \lambda}\right)}{2 \omega_{k} \sqrt{\varepsilon_{0} V}} ; \quad A_{k \lambda}^{*}=\frac{\left(\omega_{k} Q_{k \lambda}-i P_{k \lambda}\right)}{2 \omega_{k} \sqrt{\varepsilon_{0} V}}
$$

Introducing the last expressions in Eq. (17), we get the electromagnetic field energy:

$$
E_{E M}=\frac{1}{2} \sum_{k, \lambda}\left(P_{k \lambda}^{2}+\omega_{k}^{2} Q_{k \lambda}^{2}\right) \pm i \omega_{k}\left[Q_{k \lambda}, P_{k \lambda}\right]
$$

where the (+) sign is obtained when Eq. (17) is considered initially to be in the "normal order", $A_{k \lambda}^{*} A_{k \lambda}$, and the (-) one when in the "anti-normal order" $A_{k \lambda} A_{k \lambda}^{*}$.

With the purpose of establishing a harmonic oscillator representation for the electromagnetic field, it is generally considered that $\left[Q_{k \lambda}, P_{k \lambda}\right]=0$ in Eq. (26), 
because $Q_{k \lambda}$ and $P_{k \lambda}$ are simply canonical variables, getting the energy of an ensemble of harmonic oscillators:

$$
E_{E M}=\frac{1}{2} \sum_{k, \lambda}\left(P_{k \lambda}^{2}+\omega_{k}^{2} Q_{k \lambda}^{2}\right)
$$

Replacing in the last equation the classical canonical variables of position and momentum with the corresponding Hermitian operators [19, 29, 41]:

$$
\tilde{P}_{k \lambda}=i \sqrt{\frac{\hbar \omega_{k}}{2}}\left(a_{k \lambda}^{+}-a_{k \lambda}\right) ; \quad \tilde{Q}_{k \lambda}=\sqrt{\frac{\hbar}{2 \omega_{k}}}\left(a_{k \lambda}^{+}+a_{k \lambda}\right)
$$

and putting $a_{k \lambda}^{+} a_{k \lambda}=\tilde{n}_{k \lambda}$, one gets the harmonic oscillator Hamiltonian for the radiation field:

$$
\tilde{H}_{E M}=\sum_{k, \lambda}\left(\tilde{n}_{k, \lambda}+\frac{1}{2}\right) \hbar \omega_{k}
$$

At that level it is important to note that, for a harmonic oscillator of a particle with mass $m$ and momentum $\vec{p}=m d \vec{q} / d t$, with canonical variables of position $Q=|\vec{q}| \sqrt{m}$ and momentum $P=|\vec{p}| / \sqrt{m}$, the transition from the classical expression of energy:

$$
E=\frac{1}{2}\left(P^{2}+\omega^{2} Q^{2}\right)
$$

to the quantum mechanics Hamiltonian:

$$
\tilde{H}=\frac{1}{2}\left(\tilde{P}^{2}+\omega^{2} \tilde{Q}^{2}\right)=\left(a^{+} a+\frac{1}{2}\right) \hbar \omega=\left(\tilde{n}+\frac{1}{2}\right) \hbar \omega
$$

where $\tilde{P}=i \sqrt{\frac{\hbar \omega}{2}}\left(a^{+}-a\right), \tilde{Q}=\sqrt{\frac{\hbar}{2 \omega}}\left(a^{+}+a\right)$, and $\tilde{n}=a^{+} a$ is direct and needs no commutation operations between the canonical variables $P$ and $Q[19,39]$.

Consequently, the harmonic oscillator Hamiltonian for a particle of mass $m$ expressed by Eq. (31) is a quite physical result (e.g., phonons in solid-state physics) obtained with a perfect correspondence between the classical canonical variables of momentum and position $P$ and $Q$, respectively, and the corresponding Hermitian operators $\tilde{P}$ and $\tilde{Q}$.

Conversely, this is not the case for the electromagnetic field [19, 29, 39] because commutations between the canonical variables $Q_{k \lambda}$ and $P_{k \lambda}$ occur during the mathematical transition from Eq. (17) to Eq. (26). It is then considered that $\left[Q_{k \lambda}, P_{k \lambda}\right]=0$ in order to obtain Eq. (27) just before replacing the canonical variables by the corresponding quantum mechanics operators. Therein, it is important to remark that Heisenberg's commutation relation $\left[\tilde{Q}_{k \lambda}, \tilde{P}_{k^{\prime} \lambda^{\prime}}\right]=i \hbar \delta_{k k^{\prime}} \delta_{\lambda \lambda^{\prime}}$ is a fundamental concept of quantum mechanics, which should not be ignored when replacing classical variables by the corresponding quantum mechanics operators [19]. In fact, without dropping $\left[Q_{k \lambda}, P_{k \lambda}\right]$ in Eq. (26) and replacing the canonical variables by the corresponding quantum operators of Eq. (28), we get naturally the same normal ordering and anti-normal ordering radiation Hamiltonians as in Eq. (23): 


$$
\left(\begin{array}{l}
\tilde{H}_{E M}^{(+)}=\frac{1}{2} \sum_{k, \lambda}\left(\tilde{P}_{k \lambda}^{2}+\omega_{k}^{2} \tilde{Q}_{k \lambda}^{2}\right)+i \omega_{k}\left[\tilde{Q}_{k \lambda}, \hat{P}_{k^{\prime} \lambda^{\prime}}\right] \\
\tilde{H}_{E M}^{(-)}=\frac{1}{2} \sum_{k, \lambda}\left(\tilde{P}_{k \lambda}^{2}+\omega_{k}^{2} \tilde{Q}_{k \lambda}^{2}\right)-i \omega_{k}\left[\tilde{Q}_{k \lambda}, \tilde{P}_{k^{\prime} \lambda^{\prime}}\right]
\end{array}\right)=\left(\begin{array}{l}
\tilde{H}_{N O}=\sum_{k, \lambda} \tilde{n}_{k \lambda} \hbar \omega_{k} \\
\tilde{H}_{A N O}=\sum_{k, \lambda}\left(\tilde{n}_{k \lambda}+1\right) \hbar \omega_{k}
\end{array}\right)
$$

Obviously, as frequently quoted [2, 19, 39], the fundamental mathematical ambiguity consisting of cancelling the commuting classical variable term $\left[Q_{k \lambda}, P_{k \lambda}\right]=0$ before the substitution by non-commuting quantum mechanics operators $\left[\tilde{Q}_{k \lambda}, \tilde{P}_{k^{\prime} \lambda^{\prime}}\right]=i \hbar \delta_{k k^{\prime}} \delta_{\lambda \lambda^{\prime}}$ leads to the harmonic oscillator Hamiltonian for the electromagnetic field.

In fact, since no experiment has yet demonstrated that a single photon is a harmonic oscillator, the main reason for considering the electromagnetic field as an ensemble of harmonic oscillators lies in the importance of the zero-point energy (ZPE) issued in absence of photons from the eigenvalue $n_{k \lambda}=0$ of Eq. (29) corresponding to the vacuum energy:

$$
E_{Z P E}=\sum_{k, \lambda} \frac{1}{2} \hbar \omega_{k}
$$

The summation of the last expression over all modes and polarisations is infinite and represents the principal singularity in the QED formalism $[19,26,29,36,39]$.

Nevertheless, the zero-point energy is very important because it is considered to be the basis for the explanation of the vacuum effects such as the spontaneous emission, the Lamb shift and the Casimir effect. However, as pointed out by many authors $[19,26,39,41]$, it is important to underline that the explanation of the spontaneous emission and the Lamb shift in QED is not due to Eq. (33) but precisely to the commutation properties of the photon creation and annihilation operators, $a_{k \lambda}^{+}$ and $a_{k \lambda}$, respectively. It has to be emphasized that in quantum mechanics theory Eq. (33), being a constant, commutes with all Hermitian operators corresponding to physical observables and consequently has absolutely no influence to any quantum process.

Conversely, the zero-point energy expressed by Eq. (33) is useful for the explanation of the spontaneous emission and the Lamb shift in the classical description of radiation [2, 39, 47].

Regarding the Casimir effect, it is often commented that caution has to be taken concerning the interpretation of its physical origin because it has been demonstrated by different methods [48-50] that it can be easily explained using classical electrodynamics without invoking at all the zero-point energy.

Hence, in view of the above, the normal ordering Hamiltonian is the one mainly used in QED, casting aside the vacuum singularity issued from the harmonic oscillator formalism, while the zero-point energy issued from the harmonic oscillator Hamiltonian is principally useful in the classical formalism for the interpretation of the vacuum effects $[2,19,39,47]$.

\subsection{Electromagnetic field vector potential quantization in QED}

We have analysed in Section 3.1 the electromagnetic field energy quantization according to the harmonic oscillator representation. Now, we will analyse the vector potential field quantization following the second quantisation process. 
Considering the natural units $(\hbar=c=1)$, the radiation vector potential writes within the frame of the quantum field theory (QFT) [26, 38]:

$$
\vec{A}(x)=\int \frac{d^{3} k}{(2 \pi)^{3} 2 k_{0}} \sum_{\lambda=1}^{2} \hat{\varepsilon}_{k \lambda}\left[\alpha^{(\lambda)}(k) e^{-i k x}+\alpha^{(\lambda)+}(k) e^{i k x}\right]
$$

with

$$
\left[\alpha^{(\lambda)}(k), \alpha^{\left(\lambda^{\prime}\right)+}\left(k^{\prime}\right)\right]=2 k_{0}(2 \pi)^{3} \delta_{\lambda \lambda^{\prime}} \delta^{3}\left(\vec{k}-\vec{k}^{\prime}\right)
$$

where $\delta_{\lambda \lambda^{\prime}}$ is the Kronecker delta, $\delta^{3}\left(\vec{k}-\overrightarrow{k^{\prime}}\right)$ is the Dirac delta function, and

$$
\alpha^{(\lambda)}(k)=\left(2 k_{0}\right)^{1 / 2}(2 \pi)^{3 / 2} \alpha_{k \lambda} ; \quad \alpha^{(\lambda)+}(k)=\left(2 k_{0}\right)^{1 / 2}(2 \pi)^{3 / 2} \alpha_{k \lambda}^{+}
$$

Using Eq. (36) in Eq. (34), the vector potential becomes:

$$
\vec{A}(x)=\int \frac{d^{3} k}{(2 \pi)^{3 / 2}\left(2 k_{0}\right)^{1 / 2}} \sum_{\lambda=1}^{2} \hat{\varepsilon}_{k \lambda}\left[\alpha_{k \lambda} e^{-i k x}+\alpha_{k \lambda}^{+} e^{i k x}\right]
$$

with $\omega_{k}=\left(\vec{k}^{2}+l^{2}\right)^{1 / 2}$ and $k^{2}=k_{0}^{2}-(\vec{k})^{2}=l^{2}$.

For $k=l=0$ then $k_{0}=|\vec{k}|=\omega_{k}$ and Eq. (37) writes:

$$
\vec{A}(x)=\int \frac{d^{3} k}{(2 \pi)^{3 / 2}} \sqrt{\frac{1}{2 \omega_{k}}} \sum_{\lambda=1}^{2} \hat{\varepsilon}_{k \lambda}\left[\alpha_{k \lambda} e^{-i k x}+\alpha_{k \lambda}^{+} e^{i k x}\right]
$$

Suppressing the natural units (i.e., introducing $c$ and $\hbar$ ) and transforming the last equation in the SI system, which is generally used in QED, we get $[38,41,51]$.

$$
\vec{A}(x)=\int \frac{d^{3} k}{(2 \pi)^{3 / 2}} \sqrt{\frac{\hbar}{2 \varepsilon_{0} \omega_{k}}} \sum_{\lambda=1}^{2} \hat{\varepsilon}_{k \lambda}\left[\alpha_{k \lambda} e^{-i k x}+\alpha_{k \lambda}^{+}(k) e^{i k x}\right]
$$

On the basis of the density of state theory, the quantization of a field in a cavity of volume $V$ permits to transform the continuous summation over the modes to a discrete one $[19,51]$ :

$$
\int \frac{d^{3} k}{(2 \pi)^{3 / 2}}=\sqrt{\frac{1}{V}} \sum_{k}
$$

The last transformation is only valid for an ensemble of modes $k$ whose wavelengths $\lambda_{k}$ are much shorter than the characteristic dimensions of the volume $V$ $[19,29,39,41]$.

Switching now to Heisenberg's representation:

$$
\alpha_{k \lambda}(t)=\alpha_{k \lambda} e^{-i \omega_{k} t} \quad ; \quad \alpha_{k \lambda}^{+}(t)=\alpha_{k \lambda}^{+} e^{i \omega_{k} t}
$$


Generalizing the coordinate system, adapting the phase and using Eq. (40), the vector potential of the electromagnetic field writes in QED [19, 29, 39, 41, 51]:

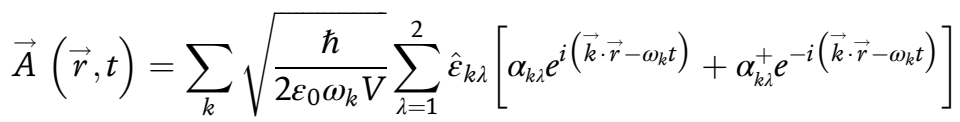

Considering the scalar potential to be constant, the electric field is:

$$
\vec{E}(\vec{r}, t)=i \sum_{k} \sqrt{\frac{\hbar \omega_{k}}{2 \varepsilon_{0} V}} \sum_{\lambda=1}^{2} \hat{\varepsilon}_{k \lambda}\left[\alpha_{k \lambda} e^{i\left(\vec{k} \cdot \vec{r}-\omega_{k} t\right)}-\alpha_{k \lambda}^{+} e^{-i\left(\vec{k} \cdot \vec{r}-\omega_{k} t\right)}\right]
$$

The last expressions represent in a given volume $V$ the quantized vector potential and the electric field of the electromagnetic radiation composed of a large number of modes $k$ each with angular frequency $\omega_{k}$ and wavelength $\lambda_{k}=2 \pi c / \omega_{k}$ much smaller than $V^{1 / 3}$ :

$$
\lambda_{k} \ll V^{1 / 3} \quad(\forall k)
$$

The amplitudes in Eqs. (42) and (43) have been obtained using the density of state theory and are valid only on the condition of Eq. (44). Furthermore, the boundary conditions of the electromagnetic waves considered in cavities and waveguides impose the wave vectors $k$ of the modes to be higher than a characteristic cut-off value $k>k_{\text {cut-off }}\left(\lambda_{k}<\lambda_{\text {cut-off }}\right)$ depending on the dimensions and the shape of the volume containing the radiation field [4-7]. Consequently, for a volume $V$ with finite dimensions, the summation in Eqs. (42) and (43) runs only over the modes $k$ with wave vectors higher than the minimum cut-off value $k_{\text {cut-off }}(V)$ imposed by the shape and dimensions of $V$ so that we can write more precisely:

$$
\begin{aligned}
& \vec{A}(\vec{r}, t)=\sum_{k>k_{\text {cut } \text { off }}(V)} \sqrt{\frac{\hbar}{2 \varepsilon_{0} \omega_{k} V} \sum_{\lambda=1}^{2} \hat{\varepsilon}_{k \lambda}\left[\alpha_{k \lambda} e^{i\left(\vec{k} \cdot \vec{r}-\omega_{k} t\right)}+\alpha_{k \lambda}^{+} e^{-i\left(\vec{k} \cdot \vec{r}-\omega_{k} t\right)}\right]} \\
& \vec{E}(\vec{r}, t)=i \sum_{k>k_{\text {cut }- \text { off }}(V)} \sqrt{\frac{\hbar \omega_{k}}{2 \varepsilon_{0} V}} \sum_{\lambda=1}^{2} \hat{\varepsilon}_{k \lambda}\left[\alpha_{k \lambda} e^{i\left(\vec{k} \cdot \vec{r}-\omega_{k} t\right)}-\alpha_{k \lambda}^{+} e^{-i\left(\vec{k} \cdot \vec{r}-\omega_{k} t\right)}\right]
\end{aligned}
$$

The last equations represent the vector potential and the electric field of a large number of modes $k$ of the quantized electromagnetic field in a finite volume $V$ with $\lambda_{k} \ll V^{1 / 3}(\forall k)$.

\section{Quantized vector potential of a single photon}

We have seen in Section 3.1 that according to the energy quantization procedure, a $k$-mode and $\lambda$-polarisation photon is considered to be a point harmonic oscillator represented by the simplified eigenfunction $\left|1_{k \lambda}\right\rangle$. On the other hand, following the field quantization procedure in Section 3.2, it appears clearly that the established vector potential and electric field expressions in Eqs. (42) and (43), 
under the condition of Eq. (44), concern a large number of modes in a considerably big volume compared to their wavelengths. Thus, with the aim of obtaining a clearer picture of the single photon, we will now complement the previous descriptions by enhancing the vector potential amplitude quantization to the photon level.

\subsection{Photon vector potential amplitude and quantization volume}

As mentioned in Section 2.2, the classical expression of the mean energy density over a period for a single electromagnetic mode $k$, represented by Eq. (13), can be considered equivalent to that for a single photon in the quantum representation, given by Eq. (14) for $N\left(\omega_{k}\right)=1$, on the condition that the volume $V$ is not arbitrary but corresponds to that defined by the boundary conditions in a cavity for the considered electromagnetic mode. In fact, the physical properties of a free photon are independent on any surrounding volume unless the characteristic dimensions of the last one are of the order of the photon wavelength [52].We recall again that the experimental evidence has shown $[19,27,29,51]$ that a single photon with angular frequency $\omega_{k}$ and wavelength $\lambda_{k}=2 \pi c / \omega_{k}$ can only be localised within a volume $V_{k}$ whose dimensions are roughly the cube of its wavelength:

$$
V_{k} \propto \lambda_{k}^{3} \quad \Rightarrow \quad V_{k} \propto \omega_{k}^{-3}
$$

From a theoretical point of view, this is also compatible with the density of state theory according to which the spatial volume corresponding to a single state of the quantized field is proportional to $\omega_{k}^{-3}[19,29,39,41]$.

On the other hand, the dimension analysis of the vector potential issued from the general solution of Maxwell's equations yields that it is proportional to an angular frequency $[5,7,9]$ :

$$
\vec{A}(\vec{r}, t)=\frac{\mu}{4 \pi} \int \frac{\vec{J}\left(\vec{r}^{\prime}, t-\frac{\left|\vec{r}-\vec{r}^{\prime}\right|}{c}\right)}{\left|\vec{r}-\vec{r}^{\prime}\right|} d^{3} r^{\prime} \propto \omega \quad \omega
$$

where $J$ is the current density $\left(\mathrm{C} \mathrm{m}^{-2} \mathrm{~s}^{-1}\right)$ and $\mu$ the magnetic permeability.

Indeed, it is well established experimentally that the energy density radiated by a dipole is proportional to $\omega^{4}$ entailing from Eq. (12) that the vector potential amplitude is normally proportional to $\omega[4,5,7]$.

This result is gauge independent since it concerns the natural units of the vector potential.

According to the previous considerations, for a free single $k$-mode photon with $\lambda$-polarisation (left or right circular), the vector potential can be written in quantum and classical formalism:

$$
\begin{array}{r}
\tilde{\alpha}_{k \lambda}(\vec{r}, t)=\alpha_{0 k}\left(\omega_{k}\right)\left[\hat{\varepsilon}_{k \lambda} \alpha_{k \lambda} e^{i\left(\vec{k} \cdot \vec{r}-\omega_{k} t\right)}+\hat{\varepsilon}_{k \lambda}^{*} \alpha_{k \lambda}^{+} e^{-i\left(\vec{k} \cdot \vec{r}-\omega_{k} t\right)}\right] \\
\overrightarrow{\alpha_{k \lambda}}(\vec{r}, t)=\alpha_{0 k}\left(\omega_{k}\right)\left[\hat{\varepsilon}_{k \lambda} e^{i\left(\vec{k} \cdot \vec{r}-\omega_{k} t\right)}+\hat{\varepsilon}_{k \lambda}^{*} e^{-i\left(\vec{k} \cdot \vec{r}-\omega_{k} t\right)}\right]
\end{array}
$$

where, following to the above analysis, the amplitude writes:

$$
\alpha_{0 k}\left(\omega_{k}\right)=|\xi| \omega_{k}
$$


with $\xi$ being a constant $[2,53-55]$.

We can evaluate $\xi[2,53]$ by using Eqs. (49) and (50) in Eq. (13) and normalising the energy to that of a single photon, $\hbar \omega_{k}$, by integrating over a wavelength along the propagation direction while taking into account the experimental results on the lateral expansion of the photon $[32-35,56]$. We get:

$$
|\xi|=\left|\frac{1}{(2 \pi)^{3 / 2}} \sqrt{\frac{\hbar}{8 \alpha \varepsilon_{0} c^{3}}}\right|=\left|\frac{\hbar}{4 \pi e c}\right|=1.747 \quad 10^{-25} \text { Volt } \mathrm{m}^{-1} \mathrm{~s}^{2}
$$

where $\alpha=e^{2} / 4 \pi \varepsilon_{0} \hbar c \approx 1 / 137.036$ is the fine structure constant and $e$ is the electron charge. Obviously, when introducing Eq. (50) in Eq. (17) for a single $k$-mode photon, an appropriate volume $V_{\mathrm{k}}$ has to be considered for the equation to hold:

$$
E_{k}=\hbar \omega_{k}=2 \varepsilon_{0} V_{k} \xi^{2} \omega_{k}^{4}
$$

Thus, the characteristic volume of a free single photon writes in agreement with Eq. (47):

$$
V_{k}=\left(\frac{\hbar}{2 \varepsilon_{0} \xi^{2}}\right) \omega_{k}^{-3}
$$

Replacing $\xi$ expressed by Eq. (51) in Eq. (53), we obtain the photon quantization volume:

$$
V_{k} \approx 4 \alpha \lambda_{k}^{3}
$$

Equations (50) and (53) express the quantized vector potential amplitude and the spatial extension of a single photon with the constant $\xi$ evaluated to be $|\xi|=\hbar / 4 \pi|e| c$.

\subsection{Photon classical-quantum (wave-particle) physical properties}

For a free $k$-mode photon, the volume $V_{k}$ corresponds to the space in which the quantized vector potential oscillates at the angular frequency $\omega_{k}$ over a period along the propagation axis generating orthogonal electric and magnetic fields whose amplitudes are, respectively:

$$
\left|\overrightarrow{\varepsilon_{k}}\right|=\left|-\partial \overrightarrow{\alpha_{k \lambda}}(\vec{r}, t) / \partial t\right| \propto|\xi| \omega_{k}^{2} ; \quad\left|\overrightarrow{\beta_{k}}\right| \propto \sqrt{\varepsilon_{0} \mu_{0}}|\xi| \omega_{k}^{2}
$$

which are independent on any external arbitrary volume parameter and are directly proportional to the square of the angular frequency $[2,54,55]$.

We can now express the quantum properties of the photon, energy, momentum, and spin by integrating the classical electromagnetic expressions over the volume $V_{k}$ and by using the vector potential amplitude obtained in Eq. (50), linking in this way the classical (wave) to the quantum (particle) representations [2]. The energy writes:

$$
E_{k}=\int_{V_{k}} 2 \varepsilon_{0} \alpha_{0 k}^{2} \omega_{k}^{2} d^{3} r=\int_{V_{k}} 2 \varepsilon_{0} \xi^{2} \omega_{k}^{4} d^{3} r=2 \varepsilon_{0} \xi^{2} \omega_{k}^{4} V_{k}=\hbar \omega_{k}
$$


With the same token considering circular polarisation $[4,5,7,9]$ for the amplitudes of the electric and magnetic fields in Eq. (55), the momentum is:

$$
\overrightarrow{p_{k}}=\int_{V_{k}} \varepsilon_{0} \overrightarrow{\varepsilon_{k \lambda}} \times \overrightarrow{\beta_{k \lambda}} d^{3} r=\varepsilon_{0}\left(\sqrt{2} \omega_{k} \alpha_{0 k}\right)\left(\sqrt{2} \omega_{k} \alpha_{0 k} / c\right) V_{k} \vec{k} /|\vec{k}|=\hbar \vec{k}
$$

According to the classical electromagnetic theory, the spin can be written through the electric and magnetic field components; hence, using again the circular polarisation, we get:

$$
|\vec{S}|=\int_{V_{k}} \varepsilon_{0}\left|\vec{r} \times\left(\overrightarrow{\varepsilon_{k \lambda}} \times \overrightarrow{\beta_{k \lambda}}\right)\right| d^{3} r=\varepsilon_{0}\left(c / \omega_{k}\right)\left(\sqrt{2} \omega_{k} \alpha_{0 k}\right)\left(\sqrt{2} \omega_{k} \alpha_{0 k} / c\right) V_{k}=\hbar
$$

where we have taken the mean value $\left\langle|\vec{r}|>_{k}=c / \omega_{k}\right.$ obtained for a single photon state [57].

The fact that the quantum properties, energy, momentum, and spin, of the photon can be expressed through the classical electromagnetic fields integrated over the volume $V_{k}$ signifies that the photon has naturally a spatial extension, and consequently when employing the term "wave-particle", one must have in mind that a single photon is a "three-dimensional particle".

We can now obtain Heisenberg's uncertainty relation for position and momentum using $V_{k}$. Indeed, replacing $V$ in Eq. (16) by $V_{k}$, we get the photon vector potential amplitude operators:

$$
\tilde{\alpha}_{0 k \lambda}=|\xi| \omega_{k} \alpha_{k \lambda} ; \quad \tilde{\alpha}_{0 k \lambda}^{*}=|\xi| \omega_{k} \alpha_{k \lambda}^{+}
$$

The corresponding position $\tilde{Q}_{k \lambda}$ and momentum $\tilde{P}_{k \lambda}$ Hermitian operators $[19,29,51]$ write:

$$
\tilde{Q}_{k \lambda}=\sqrt{\varepsilon_{0} V_{k}}\left(\tilde{\alpha}_{0 k \lambda}+\tilde{\alpha}_{0 k \lambda}^{*}\right) ; \quad \tilde{P}_{k \lambda}=-i \omega_{k} \sqrt{\varepsilon_{0} V_{k}}\left(\tilde{\alpha}_{0 k \lambda}-\tilde{\alpha}_{0 k \lambda}^{*}\right)
$$

Thus, introducing Eq. (59) in Eq. (60) and using Eq. (20) with Eq. (53), Heisenberg's commutation relation, a fundamental concept in quantum theory, results directly [2]:

$$
\left[\tilde{Q}_{k \lambda}, \tilde{P}_{k^{\prime} \lambda^{\prime}}\right]=-i \varepsilon_{0} \omega_{k^{\prime}}^{2} \omega_{k} \sqrt{V_{k} V_{k^{\prime}}}\left[\left(\xi a_{k \lambda}+\xi a_{k \lambda}^{+}\right),\left(\xi a_{k^{\prime} \lambda^{\prime}}-\xi a_{k^{\prime} \lambda^{\prime}}^{+}\right)\right]=i \hbar \delta_{k k^{\prime}} \delta_{\lambda \lambda^{\prime}}
$$

The fundamental properties of the photon, energy $E_{k}$, momentum $\vec{p}_{k}$, and wave vector $\vec{k}$, are complemented by the vector potential amplitude $\alpha_{0 k}$ expressing its electromagnetic nature:

$$
E_{k} / \hbar=\left|\overrightarrow{p_{k}}\right| c / \hbar=|\vec{k}| c=\alpha_{0 k} /|\xi|=\omega_{k}
$$

Considering Heisenberg's energy-time uncertainty principle:

$$
\delta E_{k} \delta t \geq \hbar
$$

we directly deduce from Eq. (62) the vector potential-time uncertainty:

$$
\delta \alpha_{0 k} \delta t \geq|\xi|
$$


The energy and vector potential uncertainties with respect to time are intrinsic physical properties of the wave-particle nature of the photon.

\subsection{Photon wave-particle equation and wave function}

Obviously, the photon vector potential function $\overrightarrow{\alpha_{k \lambda}}(\vec{r}, t)$ expressed in Eq. (49) satisfies the wave propagation equation in vacuum issued from Maxwell's equations:

$$
\vec{\nabla}^{2} \overrightarrow{\alpha_{k \lambda}}(\vec{r}, t)-\frac{1}{c^{2}} \frac{\partial^{2}}{\partial t^{2}} \overrightarrow{\alpha_{k \lambda}}(\vec{r}, t)=0
$$

as well as the vector potential energy (wave-particle) equation for the photon [2, 54]:

$$
i\left(\begin{array}{c}
\xi \\
\hbar
\end{array}\right) \frac{\partial}{\partial t} \overrightarrow{\alpha_{k \lambda}}(\vec{r}, t)=\left(\begin{array}{c}
\tilde{\alpha}_{0 k} \\
\tilde{H}
\end{array}\right) \overrightarrow{\alpha_{k \lambda}}(\vec{r}, t)
$$

where the vector potential operator $\tilde{\alpha}_{0 k}=-i \xi c \vec{\nabla}$ and the relativistic Hamiltonian for a massless particle $\tilde{H}=-i \hbar c \vec{\nabla}$ have the eigenvalues $\xi \omega_{k}$ and $\hbar \omega_{k}$, respectively $[2,53]$.

It is worth remarking the symmetry between the pairs $\left\{E_{k}, \hbar\right\}$ and $\left\{\alpha_{0 k}, \xi\right\}$ for a single photon characterising, respectively, the particle (energy) and electromagnetic wave (vector potential) natures, having in mind that the energy corresponds to the integration of the single-mode electromagnetic field energy density over the volume $V_{k}$.

Now, when considering the propagation of a $k$-mode photon with wavelength $\lambda_{k}$, the difficulties for defining a position operator are widely commented in the literature $[27,29,30,39-41,56]$. At the same time, the efforts for defining a wave function for the photon based on the electric and magnetic fields were rather fruitless [58-62]. It has been emphasized several times [19, 26, 27, 30, 56] that a photon cannot be localised along the propagation axis in a length shorter than the wavelength $\lambda_{k}$ and within a volume smaller than roughly $\lambda_{k}^{3}$.

In fact, from a theoretical point of view, for a photon propagating in the $z$ direction, Heisenberg's uncertainty for the position $z$ and momentum $P_{z}=\hbar k_{z}=h / \lambda_{k}$ writes:

$$
\delta z \delta P_{z} \geq h \quad \rightarrow \quad \delta z \delta\left(1 / \lambda_{k}\right) \geq 1
$$

Notice that the momentum uncertainty along the propagation axis is expressed through the uncertainty over the inverse of the wavelength.

Considering now the vector potential function with the quantized amplitude $\alpha_{0 k}=|\xi| \omega_{k}$ as a real wave function for the photon, then when a $k$-mode photon is emitted at a coordinate $z_{\mathrm{e}}$ at an instant $t_{\mathrm{e}}$ and propagates in vacuum along the $z$ axis, the probability $\Pi_{k}(z)$ to be localised at time $t$ and at the coordinate $z=z_{\mathrm{e}}+c\left(t-t_{\mathrm{e}}\right)$ corresponds to the square of the modulus of the vector potential and is consequently proportional to the square of the angular frequency $[54,55]$ :

$$
\Pi_{k}(z) \propto\left|\overrightarrow{\alpha_{k \lambda}}(z, t)\right|^{2}=\xi^{2} \omega_{k}^{2} \propto \lambda_{k}^{-2}
$$


Obviously, the shorter the wavelength of the photon, the higher the localization probability in agreement with Heisenberg's uncertainty and the experimental evidence.

\subsection{Electromagnetic field ground state, photons, and electrons-positrons}

The photon vector potential is composed of a fundamental function $\Xi_{k \lambda}$ times the angular frequency $\omega_{k}$ and writes in the classical (wave) and quantum (particle) formalisms:

$$
\begin{gathered}
\overrightarrow{\alpha_{k \lambda}}=|\xi| \omega_{k}\left[\hat{\varepsilon}_{k \lambda} e^{i\left(\vec{k} \cdot \vec{r}-\omega_{k} t+\phi\right)}+\hat{\varepsilon}_{k \lambda}^{*} e^{-i\left(\vec{k} \cdot \vec{r}-\omega_{k} t+\phi\right)}\right]=\omega_{k} \vec{\Xi}_{k \lambda}\left(\omega_{k}, \vec{r}, t\right) \\
\tilde{\alpha}_{k \lambda}=|\xi| \omega_{k}\left[a_{k \lambda} \hat{\varepsilon}_{k \lambda} e^{i\left(\vec{k} \cdot \vec{r}-\omega_{k} t+\phi\right)}+a_{k \lambda}^{+} \hat{\varepsilon}_{k \lambda}^{*} e^{-i\left(\vec{k} \cdot \vec{r}-\omega_{k} t+\phi\right)}\right]=\omega_{k} \tilde{\Xi}_{k \lambda}\left(a_{k \lambda}, a_{k \lambda}^{+}\right)
\end{gathered}
$$

In this way, the general equation for the vector potential of the electromagnetic wave considered as a superposition of plane wave modes writes:

$$
\vec{A}(\vec{r}, t)=\sum_{k, \lambda}|\xi| \omega_{k}\left[\hat{\varepsilon}_{k \lambda} e^{i\left(\vec{k} \cdot \vec{r}-\omega_{k} t+\phi\right)}+\hat{\varepsilon}_{k \lambda}^{*} e^{-i\left(\vec{k} \cdot \vec{r}-\omega_{k} t+\phi\right)}\right]=\sum_{k, \lambda} \omega_{k} \vec{\Xi}_{k \lambda}\left(\omega_{k}, \vec{r}, t\right)
$$

and that of a large number of cavity-free photons in quantum electrodynamics is:

$$
\tilde{A}=\sum_{k, \lambda}|\xi| \omega_{k}\left[a_{k \lambda} \hat{\varepsilon}_{k \lambda} e^{i\left(\vec{k} \cdot \vec{r}-\omega_{k} t+\phi\right)}+a_{k \lambda}^{+} \hat{\varepsilon}_{k \lambda}^{*} e^{-i\left(\vec{k} \cdot \vec{r}-\omega_{k} t+\phi\right)}\right]=\sum_{k, \lambda} \omega_{k} \tilde{\Xi}_{k \lambda}\left(a_{k \lambda}, a_{k \lambda}^{+}\right)
$$

According to Eqs. (55) and (62), for $\omega_{k} \rightarrow 0$ all the physical properties of the photon vanish entailing that the photon exists only for a non-zero frequency of the vector potential oscillation. However, the zero-frequency level does not correspond to perfect inexistence because the fundamental field $\Xi_{k \lambda}$ does not vanish for $\omega_{k}=0$ but reduces to $\Xi_{k \lambda}^{0}$ involving the amplitude $\xi$ and the general expression of the polarisation vectors $\hat{\varepsilon}_{k \lambda}[63,64]$ and writes in the classical and quantum representations:

$$
\vec{\Xi}_{k \lambda}^{0}=|\xi|\left[\hat{\varepsilon}_{k \lambda} e^{i \phi}+\hat{\varepsilon}_{k \lambda}^{*} e^{-i \phi}\right] \quad ; \quad \tilde{\Xi}_{k \lambda}^{0}=|\xi|\left[a_{k \lambda} \hat{\varepsilon}_{k \lambda} e^{i \phi}+a_{k \lambda}^{+} \hat{\varepsilon}_{k \lambda}^{*} e^{-i \phi}\right]
$$

The field $\Xi_{k \lambda}^{0}$ is the electromagnetic field ground state (EFGS) permeating all the space $\left(\lambda_{k} \rightarrow \infty\right)$ and having zero energy and zero vector potential as well as zero electric and magnetic fields. This physical state lies beyond the Bohm-Aharonov situation in which the energy and the electric and magnetic fields are zero but a vector potential is present in space [43]. Thus, in complete absence of energy and vector potential, the field $\Xi_{k \lambda}^{0}$ can be assimilated to a quantum vacuum component constituting the main "skeleton" of any photon which now clearly appears to be a vacuum oscillation $[2,63,64]$. 
Combination of the expression $|\xi|=\hbar / 4 \pi|e| c$ to the fine structure constant definition $\alpha=e^{2} / 4 \pi \varepsilon_{0} \hbar c$ permits to draw directly the electron-positron elementary charge $e= \pm 1.60210^{-19} \mathrm{C}$, a fundamental physical constant, which now is expressed exactly through the EFGS amplitude $\xi[64,65]$ :

$$
e= \pm(4 \pi)^{2} \alpha \frac{|\xi|}{\mu_{0}}
$$

Using again Eq. (51) and recalling that the electron mass may be written as $m_{e}=e \hbar / 2 \mu_{B}$, where $\mu_{B}$ is the Bohr magneton, we deduce that the electron mass is also expressed as a function of the EFGS amplitude $\xi$ [64]:

$$
m_{e}=2 \pi c e^{2} \frac{|\xi|}{\mu_{B}}
$$

entailing that the mass derives also from the EFGS and is proportional to the charge square.

Equations (50), (74), and (75) show the strong physical relationship between photons and electrons-positrons which are all related directly to the EFGS through the amplitude $\xi$. Obviously, photons and electrons-positrons, also probably leptonsantileptons, are issued from the same quantum vacuum field. This may be at the origin of the physical mechanism governing the photon generation during the electron-positron (and probably lepton-antilepton) annihilation and that of the electron-positron (lepton-antilepton) pair creation during the annihilation of highenergy gamma photons in the vicinity of very heavy nucleus.

\section{Conclusion}

In this chapter we have presented recent theoretical developments complementing the standard formalism with the purpose of describing a single photon state in conformity with the experiments. We resume below the principal features.

The quantization of the vector potential amplitude $\alpha_{0 k}$, a real physical entity, for a single free of cavity $k$-mode photon with angular frequency $\omega_{k}$ is expressed by $\alpha_{0 k}=|\xi| \omega_{k}$, where $|\xi|=\hbar / 4 \pi|e| c$, and leads to the establishment of a vector potential - energy (electromagnetic wave-particle) formalism (Eq. (66)) expressing the simultaneous wave-particle nature of the photon. A single photon state is a local indivisible entity of the electromagnetic field extending over a wavelength $\lambda_{k}$ and consisting of the quantized vector potential oscillating at the angular frequency $\omega_{k}$, with circular polarisation, giving birth to orthogonally oscillating electric and magnetic fields whose amplitudes are proportional to the square of the angular frequency $|\xi| \omega_{k}^{2}$ (Eq. (55)). Its lateral expansion, confirmed experimentally, yields a minimum photon volume $V_{k}$ which is proportional to $\lambda_{k}^{3}$. The quantum properties of the photon, energy, momentum, and spin are obtained directly from the classical electromagnetic expressions integrated over the volume $V_{k}$ (Eqs. (56)-(58)). It is also shown (Eq. (61)) that the Heisenberg uncertainty can be readily obtained through the use of the volume $V_{k}$.

A single photon, as a local three-dimensional entity of the electromagnetic field, is absorbed and emitted as a whole and propagates guided by the non-local vector potential function (Eq. (49)), which appears to be a natural wave function for the photon satisfying both the propagation equation (Eq. (65)) and the vector potential - energy equation (Eq. (66)). The probability for detecting a photon around 
a given point on the propagation axis is obtained by the square modulus of the vector potential and is proportional to the square of the angular frequency $\xi^{2} \omega_{k}^{2}$ (Eq. (68)) which signifies that the higher the frequency, the better the localization, in agreement with the experiments.

Finally, the electromagnetic field ground state (EFGS) at zero frequency, a real quantum vacuum component, issues naturally from the vector potential wave function putting in evidence that photons are oscillations of the vacuum field. Furthermore, the electron-positron charge and mass are directly proportional to the vector potential amplitude quantization constant showing the strong physical relationship with the photons. Obviously, the origin of the mechanisms governing the transformations of photons to electrons-positrons and inversely lies in the nature of the electromagnetic field ground state.

\section{Author details}

Constantin Meis ${ }^{1,2}$

1 National Institute for Nuclear Science and Technology, CEA - Saclay, France

2 Department of Physics, University of Versailles, UVSQ, France

*Address all correspondence to: constantin.meis@cea.fr

\section{IntechOpen}

(C) 2019 The Author(s). Licensee IntechOpen. This chapter is distributed under the terms of the Creative Commons Attribution License (http://creativecommons.org/licenses/ by/3.0), which permits unrestricted use, distribution, and reproduction in any medium, provided the original work is properly cited. (c) BY 


\section{References}

[1] Ronchi V. The Nature of Light: An Historical Survey. Cambridge (MA) USA: Harvard University Press; 1970

[2] Meis C. Light and Vacuum. 2nd ed. Singapore: World Scientific; 2017

[3] Newton I. Opticks. New York: Dover; 1952

[4] Saleh BEA, Teich MC. Fundamentals of Photonics. New York: John Wiley \& Sons; 2007

[5] Read FH. Electromagnetic Radiation. New York: John Wiley \& Sons; 1980

[6] Born M, Wolf E. Principles of Optics. Cambridge (MA) USA: Harvard University Press; 1999

[7] Jackson JD. Classical Electrodynamics. New York: John Wiley \& Sons; 1998

[8] Planck M. The Theory of Heat Radiation. New York: Dover; 1959

[9] Bransden BH, Joachain CJ. Physics of Atoms and Molecules. 2nd ed. London: Longman; 2003

[10] Mickelson AA et al. Conference on Michelson-Morley Experiment. Astrophysical Journal. 1928;68:341

[11] Hertz H. Ueber sehr schnelle electrische Schwingungen. Annalen der Physik. 1887;33:983

[12] Einstein A. In: Stachel J, Cassidy DC, Renn J, Schulmann R, editors. The collected papers of Albert Einstein. New Jersey: Princeton University Press; 1987

[13] Bohr N, Kramers HA, Slater JC. The quantum theory of radiation.

Philosophical Magazine. 1924;47:785

[14] Taylor GI. Interference fringes with feeble light. Proceedings of the
Cambridge Philosophical Society. 1909; 15:114

[15] Compton AH. A Quantum Theory of the Scattering of X-rays by Light Elements. Physical Review. 1923;21:483

[16] Wentzel G. Zur Theorie des photoelektrischen Effekts. Zeitschrift für Physik. 1926;40:574

[17] Beck G. Zur Theorie des photoeffekts. Zeitschrift für Physik. 1927;41:443

[18] Lamb WE Jr, Scully MO.

Polarization, Matière et Rayonnement,

Volume Jubilaire en L'honneur d'Alfred Kastler. Paris: French Physical Society, Press Universitaires de France; 1969

[19] Garrison JC, Chiao RY. Quantum Optics. New York: Oxford University Press; 2008

[20] Klein O, Nishina Y. über die Streuung von Strahlung durch freie Elektronen nach der neuen relativistischen. Zeitschrift für Physik. 1929;52:853

[21] Jin F, Yuan S, De Raedt H, Michielsen K, Mayiashita S. Corpuscular Model of Two-Beam Interference and Double-Slit Experiments with Single Photons. Journal of the Physical Society of Japan. 2010;79(7):074401

[22] Schawlow A, Townes C. Infrared and optical masers. Physical Review. 1958;112(6):1940-1949

[23] Klyshko DN, Penin AN, Polkovnikov BF. Parametric luminescence and light scattering by polaritons. JETP Letters. 1970;11:05

[24] Burnham DC, Weinberg DL. Observation of simultaneity in parametric production of optical photon 
pairs. Physical Review Letters. 1970; 25(2):84

[25] Ghose P, Home D. The two-prism experiment and wave-particle duality of light. Foundations of Physics. 1996;26 (7):943

[26] Akhiezer AI, Berestetskii BV. Quantum Electrodynamics. New York: Interscience; 1965

[27] Mandel L. Configuration-Space Photon Number Operators in Quantum Optics. Physical Review. 1966;144:1071

[28] Pfleegor RL, Mandel L. Interference of Independent Photon Beams. Physical Review. 1967;159(5):1084

[29] Grynberg G, Aspect A, Fabre C. Quantum Optics. New York: Cambridge University Press; 2010

[30] Auletta G. Foundations and Interpretation of Quantum Mechanics. Singapore: World Scientific; 2001

[31] Grangier P, Roger G, Aspect A. Experimental evidence for a photon anticorrelation effect on a beam splitter: A new light on single-photon interferences. Europhysics Letters. 1986;1(4):173

[32] Robinson HL. Diffraction patterns in circular apertures less than one wavelength. Journal of Applied Physics. 1953;24(1):35

[33] Hadlock RK. Diffraction Patterns at the Plane of a Slit in a Reflecting Screen. Journal of Applied Physics. 1958;29:918

[34] Hunter G, Wadlinger RLP. Photons and Neutrinos as Electromagnetic Solitons. Physics Essays. 1989;2:158

[35] Hunter G. Quantum uncertainties. NATO ASI Series B. In: Proceedings of NATO Advanced Research Workshop; June 23-27, 1986. p. 331
[36] Feynman R. The Strange Theory of Light and Matter. New Jersey: Princeton University Press; 1988

[37] Heitler W. The Quantum Theory of Radiation. Oxford: Clarendon Press; 1954

[38] Ryder LH. Quantum field theory. Cambridge: Cambridge University Press; 1987

[39] Milonni PW. The Quantum Vacuum. London: Academic Press Inc.; 1994

[40] Mittleman MH. Introduction to the Theory of Laser-Atom Interactions. New York: Plenum Press; 1982

[41] Weissbluth M. Photon-Atom Interactions. London: Academic Press Inc.; 1988

[42] Ehrenberg W, Siday RE. The refractive index in electron optics. Proceedings of the Physical Society. Section B. 1949;62:8-2

[43] Aharonov Y, Bohm D. Significance of electromagnetic potentials in the quantum theory. Physics Review. 1959; 115:485-491

[44] Chambers RG. Shift of an electron interference pattern by enclosed magnetic flux. Physical Review Letters. 1960;5:3-5

[45] Tonomura A et al. Evidence for aharonov-bohm effect with magnetic field completely shielded from electron wave. Physical Review Letters. 1986;56: 792

[46] Osakabe $\mathrm{N}$ et al. Experimental confirmation of Aharonov-Bohm effect using a toroidal magnetic field confined by a superconductor. Physical Review A. $1986 ; 34: 815$

[47] Welton TA. Physics Review. 1948; 74:1157 
[48] Milonni PW. Physical Review A. 1982;25:1315

[49] Schwinger J, DeRaad LL Jr, Milton KA. Annals of Physics. 1978;115:1

[50] Ezawa H, Nakamura K, Watanabe K. The Casimir force from Lorentz's. Frontiers in Quantum Physics. In: Lim SC, Abd-Shukor R, Kwek KH, editors. Proceedings of the 2nd International Conference; Kuala Lumpur, Malaysia; July 9-11, 1997. Springer; 1998. pp. 160-169

[51] Tannoudji CC, Diu B, Laloë F. Mécanique Quantique. Vol. III. Paris: EDP Sciences; 2017

[52] Walther H, Varcoe BTH, Englert BG, Becker T. Reports on Progress in Physics. 2006;69(5):1325

[53] Meis C. Photon wave-particle duality and virtual electromagnetic waves. Foundations of Physics. 1997;27: 865

[54] Meis C, Dahoo PR. Vector potential quantization and the photon intrinsic electromagnetic properties: Towards nondestructive photon detection. International Journal of Quantum Information. 2017;15(8):1740003

[55] Meis C, Dahoo PR. Vector potential quantization and the photon wave function. Journal of Physics: Conference Series. 2017;936:012004

[56] Jeffers S, Roy S, Vigier JP, Hunter G. The Present Status of the Quantum Theory of Light. Boston: Kluwer Academic Publishers; 1997

[57] Rybakov YP, Saha B. Soliton model of atom. Foundations of Physics. 1995; 25(12):1723-1731

[58] Bialynicki-Birula I. Photon wave function. In: Wolf E, editor. Progress in Optics XXXVI. Amsterdam: Elsevier; 1996
[59] Chandrasekar N. Quantum mechanics of photons. Advanced Studies in Theoretical Physics. 2012;6 (8):391-397

[60] Sipe JE. Photon wave functions. Physical Review A. 1995;52:1875

[61] Smith BJ, Raymer MG. Photon wave functions, wave packet quantization of light and coherence theory. New Journal of Physics. 2007;9:414

[62] Khokhlov DL. Spatial and temporal wave functions of photons. Applied Physics Research. 2010;2(2):50-54

[63] Meis C. Vector potential quantization and the quantum vacuum. Physics Research International. 2014; 2014:187432

[64] Meis C. The electromagnetic field ground state and the cosmological evolution. Journal of Physics:

Conference Series. 2018;1141:012072

[65] Meis C, Dahoo PR. The single photon state, the quantum vacuum and the elementary electron-positron charge. American Institute of Physics: Conference Proceedings. 2018;2040: 020011 
Section 2

\section{Generation}





\title{
Generation and Manipulation of Nonclassical Photon Sources in Nonlinear Processes
}

\author{
Zhi-Yuan Zhou and Bao-Sen Shi
}

\begin{abstract}
Nonclassical photon sources are key components in quantum information science and technology. Here, the basic principles and progresses for single photon generation and their further manipulation based on second- or third-order nonlinear processes in various degrees of freedom are briefly reviewed and discussed. Based on spontaneous parametric down-conversion and spontaneous four-wave mixing, various nonlinear materials such as quasi-phase-matching crystals, dispersion-shifted fibers, and silicon-on-insulator waveguides are used for single photon generation. The kinds of entanglement generated include polarization, time-energy, time-bin, and orbital angular momentum. The key ingredient for photon pair generation in nonlinear processes is described and discussed. Besides, we also introduce quantum frequency conversion for converting a single photon from one wavelength to another wavelength, while keeping its quantum properties unchanged. Finally, we give a comprehensive conclusion and discussion about future perspectives for single photon generation and manipulation in nonlinear processes. This chapter will provide an overview about the status, current challenge, and future perspectives about single photon generation and processing in nonlinear processes.
\end{abstract}

Keywords: photon pair, spontaneous parametric down-conversion, spontaneous four-wave mixing, polarization, time-bin, time-energy, orbital angular momentum, quantum entanglement, quantum frequency conversion, quasi-phase-matching, dispersion-shifted fiber, silicon-on-insulator waveguide

\section{Introduction}

Nonclassical photon sources are fundamental resources for researches in quantum information science and technology (QIST), which are widely used for applications like quantum communications, computations, sensing, and studying fundamental physics of quantum mechanics [1-3]. Therefore the ability to generate and manipulate single photon determines how far we can go in QIST. Generally, there are two distinct methods for generating single photons: one is based on excitation-reemission of photon in a semiconductor quantum dot [4], a single defect in NV center [5], or a single atom [6]; another convenient method is based on spontaneous emission based on a second- [7] or a third-order nonlinear process [8]. In this chapter, we will focus on single photon generation by using nonlinear processes. Usually, there are two nonlinear processes for generating nonclassical photon pairs: 
(1) spontaneous parametric down-conversion (SPDC), which is a second-order nonlinear process; (2) spontaneous four-wave mixing (SFWM), which is a thirdorder nonlinear process. In both SPDC and SFWM, energy, linear momentum, and angular momentum conservations should be fulfilled. Due to these conservation laws and the technology of quantum interference used, two photons in each pair generated in SPDC and SFWM can be correlated in various degrees of freedoms, for example, polarization, energy-time, orbital angular momentum, position-linear momentum, angular momentum, and photon number and path [1]; we can utilize these freedoms in a specific application scenario in QIST.

In the subsequent section, we will first introduce the basic principle of SPDC and SFWM for generating photon pairs and then the various materials used for SPDC and SFWM. In the key part of this chapter, we will review the developments of various entangled photon pair sources and methods for charactering these sources. After that we will introduce a nonlinear method for transducing the wavelength of the photon from one to another while keeping its quantum properties unchanged, which is suitable for building up a quantum interface to connect different quantum systems. Finally, we will give a brief summary in which some future perspectives for nonclassical photon pair generation and potential applications are discussed.

\section{Photon pair generation using SPDC or SFWM}

SPDC is realized in a second-order nonlinear process (see Figure 1 left image), in which a pump photon at higher frequency $\left(\omega_{p}\right)$ is split into two daughter photons at lower frequencies with certain probability in a nonlinear crystal; these two daughter photons are usually called signal $\left(\omega_{s}\right)$ and idler $\left(\omega_{i}\right)$ photons. The conservation laws of energy, linear momentum, and angular momentum require that the frequency, linear momentum $(k)$, and angular momentum $(l)$ of the pump, signal, and idler photon fulfill the following conditions: $\omega_{p}=\omega_{s}+\omega_{i} ; k_{p}=k_{s}+k_{i} ; l_{p}=l_{s}+l_{i}$. These conservation laws are responsible for the generation of various entangled sources.

In correspondence to SPDC, SFWM is a third-order nonlinear process; a big difference is that there are two pump beams in SFWM (see Figure 1 right image), in comparison to SPDC in which only one pump beam is used. The conservation laws in SFWM require that the corresponding parameters of the pump, signal, and idler photons have the following relationships: $\omega p_{1}+\omega p_{2}=\omega_{s}+\omega_{i} ; k p_{1}+k p_{2}=k_{s}+k_{i} ; l p_{1}$ $+l p_{2}=l_{s}+l_{i}$.

For quantum optical description of SPDC and SFWM, the Hamiltonian of the two processes can be expressed as [9]:

$$
\hat{H}=\hbar \xi\left(\hat{a}_{s}^{\dagger} \hat{a}_{i}^{\dagger}+\text { H.C. }\right)
$$

where $\xi$ depends on the pump intensity, the nonlinear coefficient of the crystal, crystal length, and focusing parameters. Therefore the photon states generated in SPDC and SFWM can be expressed in Fock state basis as [9]:

$$
|\Phi\rangle=\operatorname{Exp}\left[-\frac{i \hat{H} t}{\hbar}\right]|0,0\rangle=|0,0\rangle+\kappa|1,1\rangle+\kappa^{2} / 2|2,2\rangle+\ldots
$$

It can be seen from Eq. (2) that we obtain a vacuum state with a high probability if the pump is weak. The second term is the photon pair state we need, and the other terms are multiphoton states which should be avoided. It is clear from Eq. (2) that the pump beam should be at a moderate intensity level in order to eliminate the effects of higher photon number states. The photon pair generated in SPDC and SFWM is of probability and is undetermined, which is a disadvantage for photon 


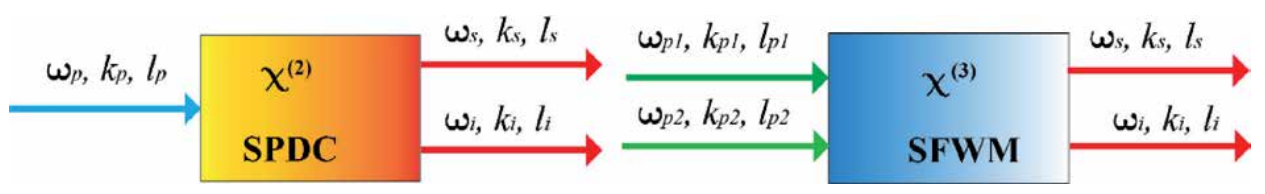

Figure 1.

A simple diagram for SPDC and SFWM. The conservation of energy, linear momentum, and angular momentum holds in both nonlinear processes.

sources generated from nonlinear processes. For this reason, one needs to choose a proper pump intensity level in order to balance between different experimental parameters.

All materials have third-order nonlinearity, but only those materials that are central asymmetric have second-order nonlinearity. The commonly used materials for SPDC can be divided into two kinds according to different phase matching: one is birefringent angle phase-matching materials, such as LBO, BBO, KTP, and LN [10]; another is quasi-phase-matching (QPM) crystals such as PPKTP and PPLN [11]. QPM crystals have the advantages of high generation rate and narrow bandwidth, which are frequently used in photon pair generation in modern quantum optics experiments [12-19]. For SFWM, the commonly used materials are hot or cold atomic ensembles [20-24] and guided-wave materials such as dispersionshifted fibers (DSF) [25-29], photonic crystal fibers (PCF), [30-32], and siliconon-insulator (SOI) waveguide [33-41]. To look for new kinds of nonlinear materials for generating high-quality photon sources is still a very hot topic in QIST.

\section{Various kinds of photon sources generated in SPDC and SFWM}

Because of the conservation of energy, linear momentum, and angular momentum in SPDC and SFWM, various kinds of nonclassical sources can be generated; in this section we will review the recent development and key points in generating various kinds of nonclassical photon sources.

\subsection{Polarization-entangled photon source}

A polarization-entangled photon source (PEPS) is one of the most important entangled photon sources that have been studied for decades of years. In the literatures, people generate PEPS using different materials with different experimental configurations [12-19, 42, 43]. For SPDC, in the early times, PEPSs are created using birefringence phase-matching (BPM) crystals, for example, a type-II phasematched BBO crystal is used to create a PEPS in the first practical and effective experiment, in which orthogonal polarized photons are emitted at the intersection cones [42]. Later on, a beam-like design is used for high-brightness photon pair generation, which is widely used in multiphoton quantum experiments [44]. The significant progress in nonlinear crystal fabrication makes a QPM crystal a better choice for researchers in many nonlinear optics applications [11]. The most important merit of using QPM crystals in generation photon pairs is its high spectral brightness in contrast to BPM crystals, due to its large effective nonlinear coefficient and long allowable interaction length.

Recently, to generate PEPS by placing a QPM crystal inside a Sagnac interferometer configuration has been demonstrated to be superior to other configurations (see Figure 2). The basic idea for a Sagnac loop-based PEPS is as the following: a pump beam is split into two beams by a double polarized beam splitter (DPBS) and 


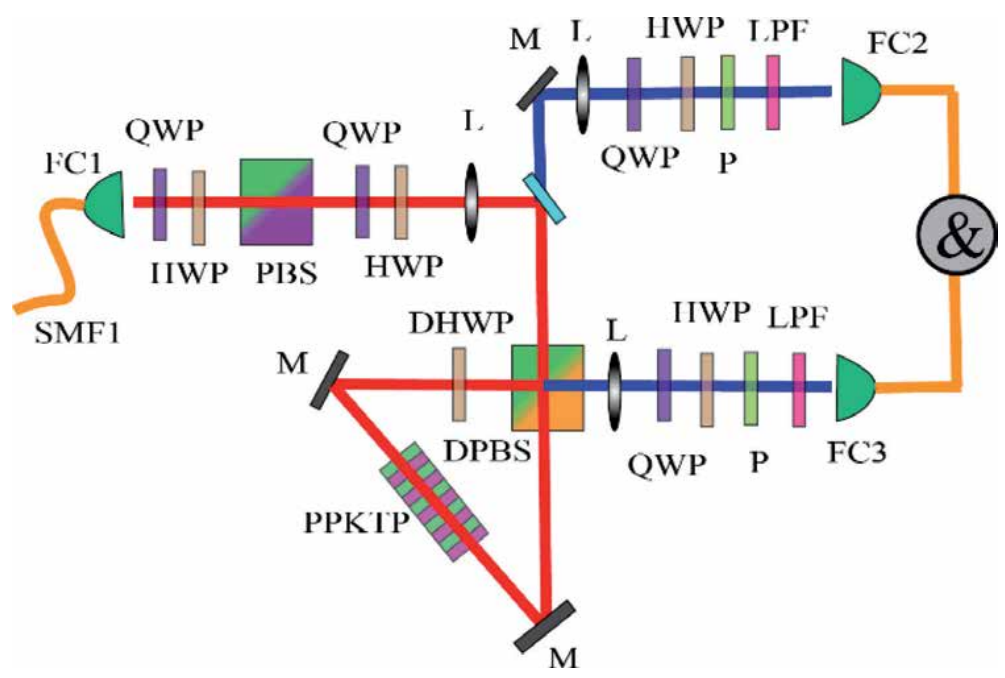

Figure 2.

The experimental setup for a typical polarization-entangled source based on Sagnac interferometer (figure cited from [13]).

counter-propagates in the Sagnac loop; each beam generates a pair of photon with orthogonal polarization, in one circulation direction; the photon pair is rotated by a double half wave plate; then two pairs of photons are recombined in the DPBS; and a PEPS with a form of $|\Phi\rangle=1 / \sqrt{2}\left(|H V\rangle+\mathrm{e}^{i \theta}|V H\rangle\right)$ is generated; the relative phase $\theta$ can be tuned by a pair of wave plates placed in the input port of the Sagnac loop. The merits to use the Sagnac interferometer configuration are its compactness, high stability, and high brightness. The original idea of Sagnac loop-based PEPS is from [43] where a BPM crystal is used, and then this idea is generalized to a QPM crystal by Kim in 2006 [45] for a CW pumped photon source. After that, a pulsed PEPS at $780 \mathrm{~nm}$ based on this configuration was developed by Kuzucu and Wong in 2008 [46]. In the early experiments, the wavelengths of the photons generated are in visible range; therefore these photons are not suitable for long-distance quantum communications in fiber. Only recently, telecom band PEPS is developed $[13,16]$. A pulsed PEPS at $1584 \mathrm{~nm}$ based on a type-II PPKTP was demonstrated by Jin et al. in 2014 [16], and Li et al. reported a tunable CW PEPS in 2015 [13]. Now, PEPS based on QPM crystals in a Sagnac configuration has become a basic tool for many experiments [47-49].

In SFWM, PEPS is generated using an atomic ensemble with different configurations. The PEPS generated with the atomic ensemble has narrow bandwidth; the wavelength is fixed to specific atomic transition lines [50,51]. Many works report PEPS generation based on guided-wave materials such as DSF $[8,25,27,28]$, PCF [30], and SOI waveguide [37, 52], the advantages of using guided-wave materials are free of free-space coupling, low loss, low cost, and easy to integrate. The guidedwave platform is very promising in large-scale applications which require hundreds of optical components. It is also convenient for building up a compact, versatile photonic source platform for various kinds of applications in QIST.

\subsection{Time-energy and time-bin-entangled photon source}

Because of conservation of energy in nonlinear processes, the two photons in each pair generated are correlated in frequency and are also generated simultaneously. Although the uncertainty in time and frequency domain for individual 
particle should meet the requirement of uncertainty principle, the sum of the frequency of signal and idler multiplies the difference between arrive times of the two photon should have a very small value, and violates an inequality for two photons existed classical correlations [53]. A two-photon Franson-type interference is used to characterize the correlations between the two photons; the phases between the two unbalanced Michelson interferometers (UMI) are correlated [54, 55]. To generate a time-energy entangled photon pair, a laser with long coherent time is needed (see Figure 3(a)); the time difference between two paths in UMI should be much larger than the coherence time of the single photon but much shorter than the coherent time of the pump laser [53]. A similar kind of entangled photon source is a time-bin entangled photon source [56], in which a pulse pump is split into two pulses in an UMI, and then these two pulses have a certain probability to generate a pair of photon separately; the photon pairs generated by these two pulses are indistinguishable after passing through two UMIs (the time difference of the UMI in measurement part is the same as the UMI in pump part, see Figure 3(b)). The quantum states for a time-energy or a time-bin entangled photon source can be expressed as $|\Phi\rangle=1 / \sqrt{2}\left(|S S\rangle+e^{i \theta}|L L\rangle\right)$, where $S$ and $L$ represent the short and long arm of the UMIs, respectively. A time-bin entangled photon pair is robust for long-distance transmission, which is widely used in demonstrating various quantum communication protocols [57]. A time-energy entangled photon source has been realized in various material systems such as atomic vapor [21], nonlinear crystals [56, 57], and guided-wave platform $[26,28,34,35,39-41]$. The differences between various materials are the photon emission bandwidth and spectral ranges. Furthermore, researchers have realized three photon genuine time-energy entangled photon sources, and their nonclassical correlations are verified [58].

\subsection{Orbital angular momentum entangled photon source}

Another important degree of freedom of photon is orbital angular momentum (OAM), which has been widely investigated since 1992 [59]. OAM has unbounded dimensions, which is very promising for high-capacity communication task in both classical and quantum optical communications [60-62]. OAM entangled photon pairs can be generated in SPDC and SFWM based on crystals [48, 63-69] and atomic vapors $[70,71]$. The quantum state for an OAM entangled photon pair generated directly by pumping a nonlinear crystal (Figure 4, left image) can be
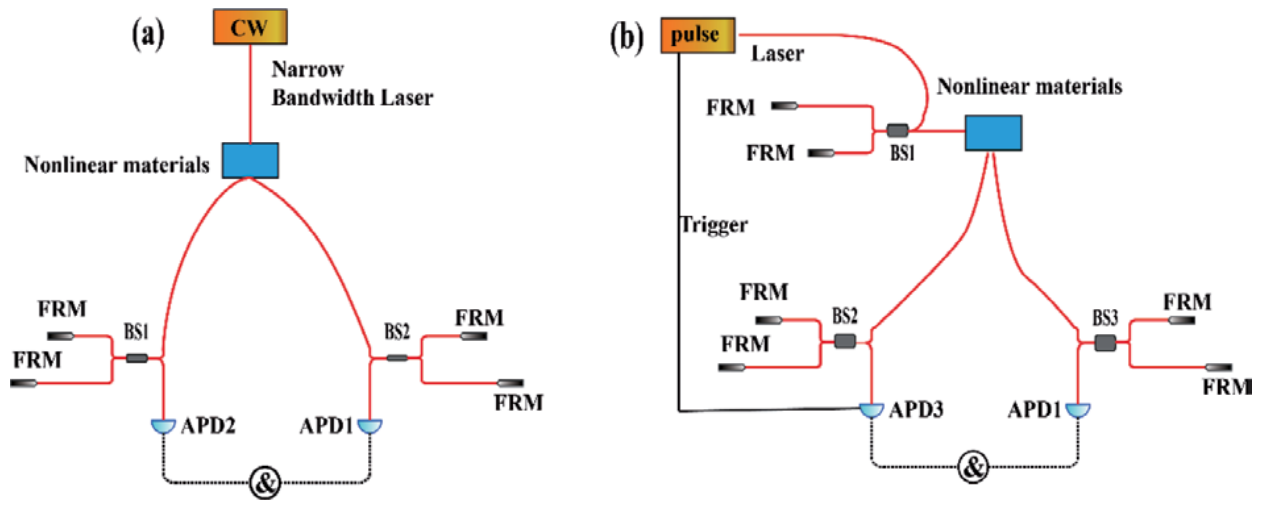

Figure 3.

Simplified diagrams for (a) time-energy; (b) time-bin entangled photon generation. A narrow bandwidth CW laser is used for generating of time-energy entangled photon pair, while a pulse laser is used for generating time-bin entangled photon pair. 


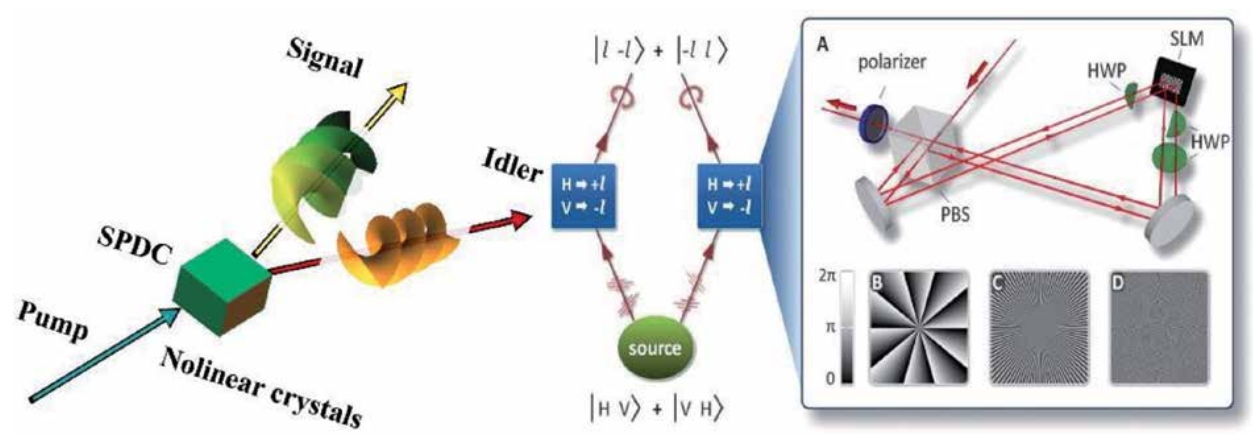

Figure 4.

Generate HD OAM entangled state directly from SPDC (left image); 2D OAM entangled state generation by converting a polarized entangled state into $2 D$ subspace of OAM entangled state (right image, cited from [48]).

expressed as $|\Phi\rangle=\sum C_{l}|l,-l\rangle$, where $C_{l}$ is the weight for different OAM modes. One can investigate OAM entanglement in a two-dimensional (2D) subspace [48, 63, 67-70] or in high-dimensional (HD) space $[65,66,71]$. The properties and the methods of characterizing a $2 \mathrm{D}$ entangled source in different degrees of freedom are similar and can be converted from one kind to another [48, 68] (please see Figure 4 (right images)). The post-selected OAM entangled states in a 2D subspace can be expressed as $|\Phi\rangle=1 / \sqrt{2}(|l,-l\rangle+|-l, l\rangle)$. While for a HD entangled source, the properties and the methods of characterization are rather different. [65] reported on the realization of a 11D entangled source, demonstrating the violation of the Bell inequality. Zeilinger's group has demonstrated a 100*100 HD entanglement by measuring the entanglement witness of the generated state [66]. For a 2D OAM entangled photon source, Zeilinger's group converted a polarized entangled photon source into an OAM entangled source with OAM momenta of $300 \mathrm{~h}$ in 2D subspace via a spatial light modulator [48]. Later on, a higher OAM momentum of about 10,000 h for a 2D OAM entangled source is realized by using a vortex reflection mirror [68]. A HD OAM entangled photon source is preferred for studying the basic principle of quantum mechanics and for HD quantum communication applications.

\section{Methods for characterizing the properties of a nonclassical photon source}

Nonclassical photon sources can be characterized from different aspects. For characterizing the properties of a heralded single photon, the heralded efficiency [72], the coincidence to accidental coincidence ratio (CAR) [73], and the single photon Glauber function $[74,75]$ are important parameters. The heralded efficiency is the probability of detecting the second photon when the first photon is detected. It is a measurement of the photon collection efficiency, filter and transmission losses, and the single photon detector efficiency. The heralded efficiency is the ratio of the coincidence count to the single count rate of the first detected photon. CAR is a measurement of the signal to background noise ratio for a two-photon experiment; high CAR can ensure the quantum nature existed between the two photons. CAR depends on pump power and detector performance. Usually, CAR will increase when the pump power is increased in the low pump power regime. After reaching the maximum value, CAR will decrease with the increase of the pump power [76]. The single photon Glauber function can be measured as shown in [74]. The measured photon is firstly split by a beam splitter, and then by measuring the three party coincidence, single count and two-photon coincidence, we can 


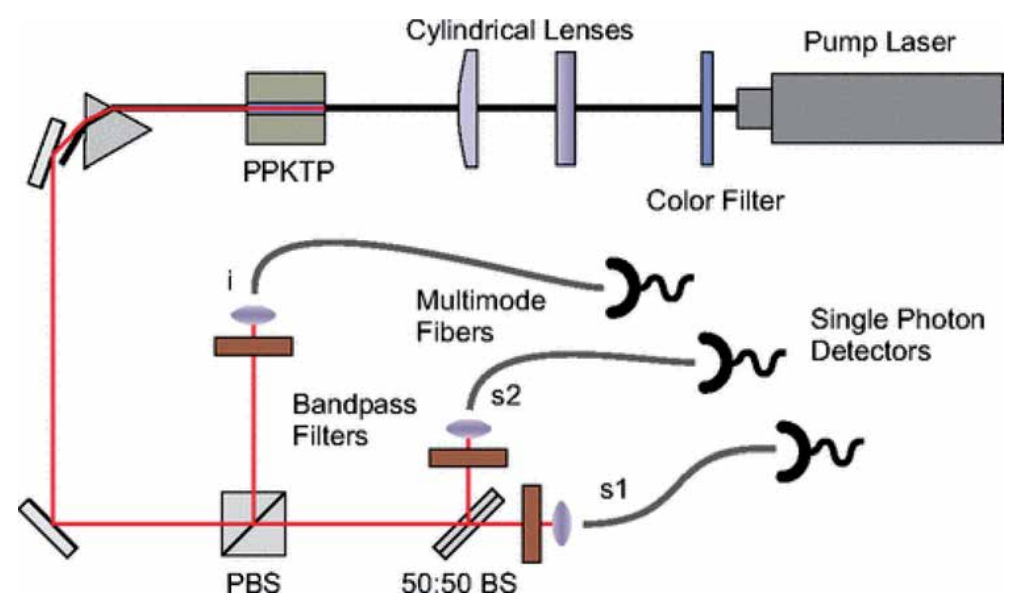

Figure 5.

Experimental setup for measuring heralded single photon autocorrelation function for single photon generated from SPDC (image cited from [74]).

calculate the single photon $g^{(2)}$ function (see Figure 5). A near zero $g^{(2)}(0)$ indicates the high quality of single photon nature. For a pulse pumped photon source, the single photon purity is also an important parameter [17, 77]. The purity of photon is a measurement of spectral correlations between two photons; the purity is determined by the Schmitt number in the Schmitt decomposition. The unity single photon purity indicates that the two-photon spectral can be factorized into product of two separate functions of the signal and idler photons. The high single photon purity is very important for realizing high visibility HOM interference between two independent single photon sources, which is the key technique for realizing high photon number entangled states.

There are various available and faithful methods to characterize the quality of entanglement of an entangled two-photon source, including two-photon interference fringes $[65,78]$, Bell CHSH inequality $[79,80]$, and quantum state tomography (QST) [81]. Two-photon interference fringe is much easier to measure; through calculating the interference visibility from the measured data, we can evaluate the quality of an entangled source. A high visibility indicates a high quality of the generated state by comparing the ideal maximum Bell states. When the visibility is greater than a threshold value, the two photons have Bell nonlocality; the threshold value is different for two-photon states in different dimension. For two photons existing in classical correlation, the Bell CHSH parameter $S$ is not greater than a certain value. The violation of this value indicates a nonclassical correlation between the twophoton states. Bell CHSH parameter $S$ is an indicator of whether the two-photon state has Bell nonlocality and how strong this kind of nonlocal correlation is. The violation of Bell inequality has been widely studied in literatures for a $2 \mathrm{D}$ and a HD entangled state. To fully know the content of a generated quantum state, QST can be used to reconstruct the density matrix of a certain quantum state. By the density matrix of a quantum state, all the properties of the quantum state can be predicted.

\section{Quantum frequency conversion for nonclassical quantum state}

There are many quantum systems for QIST based on different materials, including atomic ensembles, trapped ions, solid-state materials, and fibers for transmission [82-86]. Each quantum system has some advantages in QST, and these 


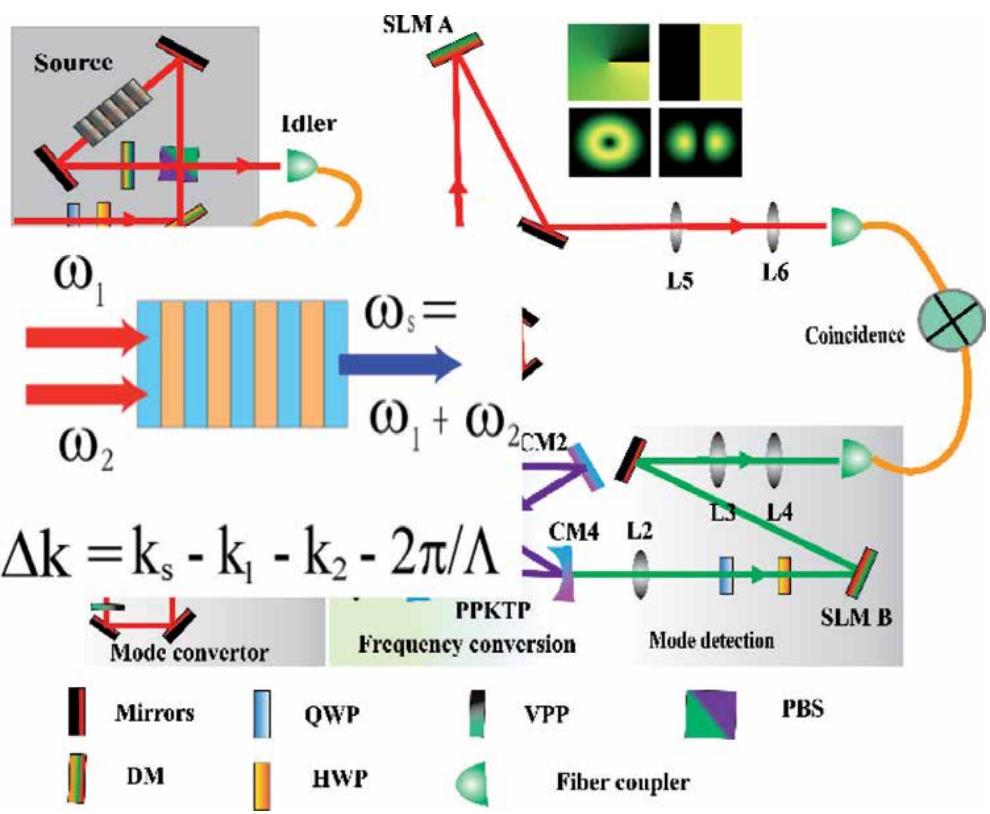

Figure 6.

Left image: simple diagram for sum frequency generation in QPM crystal. Right image: experimental setup of QFC for an OAM qubit, an OAM-polarization hybrid entangled state, and an OAM-OAM entangled state (cited from [99]).

systems usually work at different frequencies, which may have a big frequency mismatching. To build up a quantum network consisting of various quantum systems for information encoding, storage, transmission, and processing, a quantum frequency converter (QFC) to link different quantum systems is indispensable. Such a frequency transducer can be realized by utilizing nonlinear processes such as sum frequency generation (SFG) and Bragg reflection in four-wave mixing. The theory of quantum frequency conversion for SFG was first proposed by P. Kumar in 1990 [87]. In SFG, a strong pump laser can convert a weak signal beam with high quantum efficiency; the unity quantum efficiency can be reached when the pump beam is strong enough (see Figure 6 left image), and the quantum correlations are unchanged after frequency conversion. Since it has been proposed, some significant progresses have been made in this field; researchers have realized that frequency up-conversion and down-conversion for a single photon generated from quantum dot, and various qubit states or entangled states such as polarization, time-energy, and OAM entangled state [39, 88-101] have been up- or downconverted. A typical setup for QFC of an OAM qubit, an OAM-polarization hybrid entangled state, and an OAM-OAM entangled state is shown in Figure 6 (right image). For frequency down-conversion, the visible single photons generated from atomic ensemble, trapped irons, or NV centers have been converted to telecom band successfully [102-105]. In all these demonstrations, the single photon properties and entanglement are preserved in the conversion processes, which ensures that quantum information can be coupled to different quantum systems by using a quantum frequency interface.

In QFC, there are four parameters to evaluate the quality of a converter: quantum conversion efficiency, noise level, spectral bandwidth, and spatial bandwidth. These parameters are not independent; therefore in practical applications, one needs to balance between different parameters [98]. A longer nonlinear crystal is preferred to reach maximum conversion efficiency when the pump power is 
limited, but a longer nonlinear crystal would lead to a smaller spectral bandwidth and spatial bandwidth; a proper crystal length should be chosen to balance conversion efficiency and spectral bandwidth. The noise in QFC comes from SPDC and SRS of the intense pump beam; therefore a longer pump wavelength is preferred to reduce the noise photon in QFC [106]. The noise photon can also be dramatically reduced by using a narrow bandwidth filters to filter out the converted photon.

\section{Discussions and conclusion}

Nonclassical photon sources are used in almost all fields of QIST; the ability to generate and control its properties is at the heart for applications in QIST. Though many progresses have been made in single photon generation and manipulation in nonlinear processes, lots of further techniques should be developed to harness the quality of single photon generated in SPDC or SFWM. The detailed techniques for optimizing the parameter of the photon source depend on the specific applications. For a pulsed heralded single photon source, the heralded efficiency and total photon count are important parameters, but the probability of single photon generation per pulse is very low, which limits the flux of photon pair generation. These defects can be overcome by using time, frequency, and OAM multiplexing to enhance the photon generation probability per pulse and total count rate [41, 107-112]. When the optical elements for multiplexing have low losses, the heralded efficiency and rate can be increased substantially [112]. For applications taking advantages of the sharp time correlations in SPDC, a broadband spectrum of the photon pair is needed. Such a broadband photon pair can be realized with an ultrathin nonlinear crystal or using a chirp quasi-phase-matching crystal; the bandwidth of the photon pair generated can be greater than $100 \mathrm{~nm}$, which has a time correlation of sub-femtosecond [113]. For quantum information applications, a multiplexed time-energy and polarized entangled photon pair is preferred for high-capacity quantum communication by using dense-wave division multiplexing technique. The multiplexed entangled sources are easier to be realized using a waveguide platform such as a PPLN waveguide, a DSF, or SOI waveguide. A SOI ring cavity is also preferred in generating frequency comb entangled states [114]. For generating HD entangled states, by shaping the profile of a pump beam, a much greater Hilbert space can be reached $[115,116]$. For QFC of OAM entangled states, the mode-dependent conversion efficiency has not been solved yet. We recently proposed and demonstrated that if we use a flat-top beam to pump the SFG crystal, then we can solve the problem of mode-dependent conversion efficiency by using a Gaussian pump beam [117].

For a compact application, integrated optics will offer a great advantage over free-space implementation; the trends of modern optics are to convert a freespace module to an equivalent integrated optical device, which will be of high compact, robust to environment fluctuations and much easier for larger amounts of fabrication $[118,119]$.

In conclusion, most of the advances and progresses for generation and manipulation of single photon sources in nonlinear processes are briefly reviewed in this chapter; this review will provide a glance at the current status, and challenges remain to be solved in this field. The general properties for single photon generation in nonlinear processes are introduced firstly; then we introduce the development of various entangled states and the methods to characterize nonclassical photonic states. Next, we review the progresses for frequency conversion of a photonic state in nonlinear processes. Finally, we give comprehensive discussions about 
the remaining challenges in generating high-quality and HD entangled states, the unsolved problems for QFC of HD OAM photonic states, and the development of integrated optics for small footprint optical devices and large-scale quantum information processing on chip. This book chapter should be helpful for new researchers working in this field.

\section{Author details}

Zhi-Yuan Zhou* and Bao-Sen Shi

Key Laboratory of Quantum Information, CAS, University of Science and Technology of China, Hefei, Anhui, China

*Address all correspondence to: zyzhouphy@ustc.edu.cn

\section{IntechOpen}

(C) 2019 The Author(s). Licensee IntechOpen. This chapter is distributed under the terms of the Creative Commons Attribution License (http://creativecommons.org/licenses/ by/3.0), which permits unrestricted use, distribution, and reproduction in any medium, provided the original work is properly cited. (cc) BY 


\section{References}

[1] Flamini F, Spagnolo N,

SciarrinoF.Photonicquantuminformation processing: A review. Reports on Progress in Physics. 2019;82:016001.

DOI: $10.1088 / 1361-6633 / a a d 5 b 2$

[2] Pan JW, Chen ZB, Lu CY, Weinfurter $\mathrm{H}$, Zeilinger A, Zukowski M. Multiphoton entanglement and interferometry. Reviews of Modern Physics. 2012;84:777-838. DOI: 10.1103/RevModPhys.84.777

[3] Kimble J. The quantum internet. Nature. 2008;453:1023-1030. DOI: 10.1038 /nature07127

[4] Lodahl P. Quantum-dot based photonic quantum networks. Quantum Science and Technology. 2018;3:013001. DOI: 10.1088/2058-9565/aa91bb

[5] Kurtsiefer C, Mayer S, Zarda P, Weinfurter H. Stable solid-state source of single photons. Physical Review Letters. 2000;85:290. DOI: 10.1103/ PhysRevLett.85.290

[6] Kuhn A, Hennrich M, Rempe G. Deterministic single-photon source for distributed quantum networking. Physical Review Letters. 2002;89:067901. DOI: 10.1103/ PhysRevLett.89.067901

[7] Burnham DC, Weinberg DL. Observation of simultaneityinparametric production of optical photon pairs. Physical Review Letters. 1970;25:84. DOI: 10.1103/PhysRevLett.25.84

[8] Takesue H, Inoue K. Generation of polarization-entangled photon pairs and violation of Bell's inequality using spontaneous four-wave mixing in a fiber loop. Physical Review A. 2004;70:031802. DOI: 10.1103/PhysRevA.70.031802

[9] Hiesmayr BC, de Dood MJA, Löffler W. Observation of four-photon orbital angular momentum entanglement. Physical Review Letters. 2016;116:073601. DOI: 10.1103/ PhysRevLett.116.073601

[10] Boyd RW. Nonlinear Optics. 3rd ed. Chichester: Academic Press; 2008. 640 p

[11] Armstrong JA, Bloembergen N, Ducuing J, Perhsan PS. Interactions between light waves in a nonlinear dielectric. Physics Review. 1962;127:1918. DOI: 10.1103/ PhysRev.127.1918

[12] Jin RB, Shimizu R, Kaneda F, Mitsumori Y, Kosaka H, Edamatsu K. Entangled-state generation with an intrinsically pure single-photon source and a weak coherent source. Physical Review A. 2013;88:012324. DOI: 10.1103/PhysRevA.88.012324

[13] Li Y, Zhou ZY, Ding DS, Shi BS. CW-pumped telecom band polarization entangled photon pair generation in a Sagnac interferometer. Optics Express. 2015;23:28792-28800.

DOI: 10.1364/OE.23.028792

[14] Zhou ZY, Jiang YK, Ding DS, Shi BS, Guo GC. Actively switchable nondegenerate polarization-entangled photon-pair distribution in dense wave-division multiplexing. Physical Review A. 2013;87:045806. DOI: 10.1103/PhysRevA.87.045806

[15] Zhou ZY, Jiang YK, Ding DS, Shi BS. An ultra-broadband continuously-tunable polarizationentangled photon-pair source covering the $\mathrm{C}+\mathrm{L}$ telecom bands based on a single type-II PPKTP crystal. Journal of Modern Optics. 2013;60:720-725. DOI: 10.1080/09500340.2013.807363

[16] Jin RB, Shimizu R, Wakui K, Fujiwara M, Yamashita T, Miki S, et al. Pulsed Sagnac polarization-entangled photon source with a PPKTP crystal at 
telecom wavelength. Optics Express. 2014;22:11498-11507. DOI: 10.1364/ OE.22.011498

[17] Jin RB, Shimizu R, Wakui K, Benichi H, Sasaki M. Widely tunable single photon source with high purity at telecom wavelength. Optics Express. 2013;21:10659-10666. DOI: 10.1364/ OE.21.010659

[18] Hentschel M, Hubel H, Poppe A, Zeilinger A. Three-color Sagnac source of polarization-entangled photon pairs. Optics Express. 2009;17:23153-23159. DOI: 10.1364/OE.17.023153

[19] Steinlechner F, Ramelow S, Jofre M, Gilaberte M, Jennewein T, Torres JP, et al. Phase-stable source of polarization-entangled photons in a linear double-pass configuration. Optics Express. 2013;21:11943-11951. DOI: 10.1364/OE.21.011943

[20] Ding DS, Zhou ZY, Shi BS, Zou XB, Guo GC. Generation of non-classical correlated photon pairs via a laddertype atomic configuration: Theory and experiment. Optics Express. 2012;20:11433-11444. DOI: 10.1364/ OE.20.011433

[21] Park J, Jeong T, Kim H, Moon HS. Time-energy entangled photon pairs from Doppler-broadened atomic ensemble via collective twophoton coherence. Physical Review Letters. 2018;121:263601. DOI: 10.1103/ PhysRevLett.121.263601

[22] Shu C, Chen P, Chow TKA, Zhu L, Xiao Y, Loy MMT, et al. Subnatural-linewidth biphotons from a Doppler-broadened hot atomic vapour cell. Nature Communications. 2016;7:12783. DOI: 10.1038/ ncomms 12783

[23] Qian P, Gu Z, Cao R, Wen R, Ou ZY, Chen JF, et al. Temporal purity and quantum interference of single photons from two independent cold atomic ensembles. Physical Review Letters. 2016;117:013602. DOI: 10.1103/ PhysRevLett.117.013602

[24] Lee JC, Park KK, Zhao TM, Kim YH. Einstein-Podolsky-Rosen entanglement of narrow-band photons from cold atoms. Physical Review Letters. 2016;117:250501. DOI: 10.1103/ PhysRevLett.117.250501

[25] Li X, Voss PL, Sharping JE, Kumar P. Optical-fiber source of polarizationentangled photons in the $1550 \mathrm{~nm}$ telecom band. Physical Review Letters. 2005;94:053601. DOI: 10.1103/ PhysRevLett.94.053601

[26] Takesue H, Inoue K. Generation of $1.5 \mu \mathrm{m}$ band time-bin entanglement using spontaneous fiber four-wave mixing and planar light-wave circuit interferometers. Physical Review A. 2005;72:041804. DOI: 10.1103/PhysRevA.72.041804

[27] Zhou Q, Zhang W, Wang P, Huang Y, Peng J. Polarization entanglement generation at $1.5 \mu \mathrm{m}$ based on walk-off effect due to fiber birefringence. Optics Letters. 2012;37:1679-1681. DOI: 10.1364/ OL.37.001679

[28] Li YH, Zhou ZY, Xu ZH, Xu LX, Shi BS, Guo GC. Multiplexed entangled photon-pair sources for all-fiber quantum networks. Physical Review A. 2016;94:043810. DOI: 10.1103/PhysRevA.94.043810

[29] Li YH, Xu ZH, Wang S, Xu LX, Zhou ZY, Shi BS. Hong-Ou-Mandel interference between two independent all-fiber multiplexed photon sources. Acta Physica Sinica. 2017;66:120302. DOI: 10.7498/aps.66.120302

[30] Fulconis J, Alibart O, O’Brien JL, Wadsworth WJ, Rarity JG. Nonclassical interference and entanglement generation using a photonic crystal fiber pair photon source. Physical Review 
Letters. 2007;99:120501. DOI: 10.1103/

PhysRevLett.99.120501

[31] Cohen O, Lundeen JS, Smith BJ, Puentes G, Mosley PJ, Walmsley IA. Tailored photon-pair generation in optical fibers. Physical Review Letters. 2009;102:123603. DOI: $10.1103 /$

PhysRevLett.102.123603

[32] Cui L, Li X, Zhao N. Spectral properties of photon pairs generated by spontaneous four-wave mixing in inhomogeneous photonic crystal fibers. Physical Review A. 2012;85:023825. DOI: 10.1103/PhysRevA.85.023825

[33] Silverstone JW, Bonneau D, Ohira K, Suzuki N, Yoshida H, Iizuka N, et al. On-chip quantum interference between silicon photon-pair sources. Nature Photonics. 2014;8:104-108. DOI: 10.1038/nphoton.2013.339

[34] Reimer C, Kues M, Roztocki P, Wetzel B, Grazioso F, Little BE, et al. Generation of multiphoton entangled quantum states by means of integrated frequency combs. Science. 2016;351:1176-1180. DOI: 10.1126/ science.aad8532

[35] Mazeas F, Traetta M, Bentivegna M, Kaiser F, Aktas D, Zhang W, et al. High quality entanglement on a chip-based frequency comb. Optics Express. 2016;24:28731-28738. DOI: $10.1364 /$ OE.24.028731

[36] He J, Bell BA, Casas-bedoya A, Zhang Y, Clark AS, Xiong C, et al. Ultracompact quantum splitter of degenerate photon pairs. Optica. 2015;2:779-782. DOI: 10.1364/ OPTICA.2.000779

[37] Li YH, Zhou ZY, Feng LT, Fang WT, Liu SL, Liu SK, et al. On-chip multiplexed multiple entanglement sources in a single silicon nanowire. Physical Review Applied. 2017;7:064005. DOI: 10.1103/ PhysRevApplied.7.064005
[38] Preble SF, Fanto ML, Steidle JA, Tison CC, Howland GA, Wang Z, et al. On-chip quantum interference from a single silicon ring-resonator source. Physical Review Applied. 2015;4:021001. DOI: 10.1103/

PhysRevApplied.4.021001

[39] Li YH, Fang WT, Zhou ZY, Liu SL, Liu SK, Xu ZH, et al. Quantum frequency conversion for multiplexed entangled states generated from micro-ring silicon chip. Optics Express. 2018;26:28429-28440. DOI: $10.1364 /$ OE.26.028429

[40] Grassani D, Azzini S,

Liscidini M, Galli M, Strain MJ, Sorel M, et al. Micrometer-scale integrated silicon source of time-energy entangled photon. Optica. 2015;2:88-94. DOI: 10.1364/OPTICA.2.000088

[41] Fang WT, Li YH, Zhou ZY, Xu LX, Guo GC, Shi BS. On-chip generation of time-and wavelengthdivision multiplexed multiple timebin entanglement. Optics Express. 2018;26:12912-12921. DOI: 10.1364/ OE.26.012912

[42] Kwiat PG, Mattle K, Weinfurter H, Zeilinger A, Sergienko AV, Shih Y. New high-intensity source of polarizationentangled photon pairs. Physical Review Letters. 1995;75:4337-4341. DOI: 10.1103/PhysRevLett.75.4337

[43] Shi BS, Tomita A. Generation of a pulsed polarization entangled photon pair using a Sagnac interferometer. Physical Review A. 2004;69:013803. DOI: 10.1103/PhysRevA.69.013803

[44] Zhang C, Huang YF, Wang Z, Liu BH, Li CF, Guo GC. Experimental Greenberger-Horne-Zeilinger-type sixphoton quantum nonlocality. Physical Review Letters. 2015;115:260402. DOI: 10.1103/PhysRevLett.115.260402

[45] Kim T, Fiorentino M, FNC W. Phase-stable source of polarization 
entangled photons using a polarization Sagnac interferometer. Physical Review A. 2006;73:012316. DOI: 10.1103/PhysRevA.73.012316

[46] Kuzucu O, FNC W. Pulsed Sagnac source of narrow-band polarizationentangled photons. Physical Review A. 2008;77:032314. DOI: 10.1103/PhysRevA.77.032314

[47] Giustina M, Mech A, Ramelow S, Wittmann B, Kofler J, Beyer J, et al. Bell violation using entangled photons without the fair-sampling assumption. Nature. 2013;497:227-230. DOI: 10.1038/nature12012

[48] Fickler R, Lapkiewicz R, Plick WN, Krenn M, Schaeff C, Ramelow S, et al. Quantum entanglement of high angular momenta. Science. 2012;338:640-643. DOI: $10.1126 /$ science. 1227193

[49] Hu MJ, Zhou ZY, Hu XM, Li CF, Guo GC, Zhang YS. Observation of nonlocality sharing among three observers with one entangled pair via optimal weak measurement. npj Quantum Information. 2018;4:63. DOI: 10.1038/ s41534-018-0115-x

[50] Park J, Kim H, Moon HS.

Polarization-entangled photons from a warm atomic ensemble using a

Sagnac interferometer. Physical Review Letters. 2019;122:143601. DOI: 10.1103/ PhysRevLett.122.143601

[51] Yu YC, Ding DS, Dong MX, Shi S, Zhang W, Shi BS. Self-stabilized narrowbandwidth and high-fidelity entangled photons generated from cold atoms. Physical Review A. 2018;97:043809. DOI: 10.1103/PhysRevA.97.043809

[52] Takesue H, Fukuda H, Tsuchizawa T, Watanabe T, Yamada K, Tokura Y, et al. Generation of polarization entangled photon pairs using silicon wire waveguide. Optics Express. 2008;16:5721-5727. DOI: 10.1364/ OE.16.005721
[53] Franson JD. Bell inequality for position and time. Physical Review Letters. 1989;62:2205. DOI: 10.1103/ PhysRevLett.62.2205

[54] Franson JD. Two-photon interferometry over large distances. Physical Review A. 1991;44:4552. DOI: 10.1103/PhysRevA.44.4552

[55] Ou ZY, Zou XY, Wang LJ, Mandel L. Observation of nonlocal interference in separated photon channels. Physical Review Letters. 1990;65:321. DOI: 10.1103/ PhysRevLett.65.321

[56] Brendel J, Gisin N, Tittel W, Zbinden H. Pulsed energy-time entangled twin-photon source for quantum communication. Physical Review Letters. 1999;82:2594. DOI: 10.1103/PhysRevLett.82.2594

[57] Tittel W, Brendel J, Zbinden H, Gisin N. Quantum cryptography using entangled photons in energy-time bell states. Physical Review Letters. 2000;84:4737. DOI: 10.1103/

PhysRevLett.84.4737

[58] Shalm LK, Hamel DR, Yan Z, Simon C, Resch KJ, Jennewein T. Threephoton energy-time entanglement. Nature Physics. 2013;9:19-22. DOI: $10.1038 /$ nphys 2492

[59] Allen L, Beijersbergen MW, Spreeuw RJC, Woerdman JP. Orbital angular momentum of light and the transformation of LaguerreGaussian laser modes. Physical Review A. 1992;45:8185-8189. DOI: 10.1103/PhysRevA.45.8185

[60] Yao AM, Padgett MJ. Orbital angular momentum: Origins, behavior and applications. Advances in Optics and Photonics. 2011;3:161-244. DOI: 10.1364/AOP.3.000161

[61] Wang J, Yang JY, Fazal IM, Ahmed N, Yan Y, Huang H, et al. 
Terabit free-space data transmission employing orbital angular momentum multiplexing. Nature Photonics. 2012;6:488-496. DOI: $10.1038 /$ nphoton.2012.138

[62] Barreiro JT, Wei TC, Kwiat PG. Beating the channel capacity limit for linear photonic superdense coding. Nature Physics. 2008;4:282-286. DOI: 10.1038/nphys919

[63] Mair A, Vaziri A, Weihs G, Zeilinger A. Entanglement of the orbital angular momentum states of photons. Nature. 2001;412:313-316. DOI: $10.1038 / 35085529$

[64] Leach J, Jack B, Romero J, Jha AK, Yao AM, Franke-Arnold S, et al. Quantum correlations in optical angleorbital angular momentum variables. Science. 2010;329:662-665. DOI: 10.1126/science. 1190523

[65] Dada AC, Leach J, Buller GS, Padgett MJ, Andersson E. Experimental high-dimensional two-photon entanglement and violations of generalized Bell inequalities. Nature Physics. 2011;7:677-680. DOI: 10.1038/ nphys1996

[66] Krenn M, Huber M, Fickler R, Lapkiewicz R, Ramelow S, Zeilinger A. Generation and confirmation of a $(100 \times 100)$-dimensional entangled quantum system. PNAS. 2014;111:6243-6247. DOI: 10.1073/ pnas.1402365111

[67] Leach J, Jack B, Romero J, Ritsch-Marte M, Boyd RW, Jha AK, et al. Violation of a Bell inequality in two-dimensional orbital angular momentum state-spaces. Optics Express. 2009;17:8287-8293. DOI: 10.1364/OE.17.008287

[68] Fickler R, Campbell G, Buchler B, Lam PK, Zeilinger A. Quantum entanglement of angular momentum states with quantum numbers up to
10,010. PNAS. 2016;113:13642-13647.

DOI: $10.1073 /$ pnas. 1616889113

[69] Zhou ZY, Li Y, Ding DS, Zhang W, Shi S, Shi BS. Classical to quantum optical network link for orbital angular momentum-carrying light. Optics Express. 2015;23:18435-18444. DOI: 10.1364/OE.23.018435

[70] Ding DS, Zhang W, Zhou ZY, Shi S, Xiang GY, Wang XS, et al. Quantum storage of orbital angular momentum entanglement in an atomic ensemble. Physical Review Letters. 2015;114:050502. DOI: 10.1103/ PhysRevLett.114.050502

[71] Ding DS, Zhang W, Shi S, Zhou ZY, Li Y, Shi BS, et al. High-dimensional entanglement between distant atomicensemble memories. Light: Science and Applications. 2016;5:e16157. DOI: 10.1038/lsa.2016.157

[72] Fasel S, Alibart O, Tanzilli S, Baldi P, Beveratos A, Gisin N, et al. High-quality asynchronous heralded single-photon source at telecom wavelength. New Journal of Physics. 2014;6:163. DOI: 10.1088/1367-2630/6/1/163

[73] Chen J, Lee KF, Liang C, Kumar P. Fiber-based telecom-band degenerate-frequency source of entangled photon pairs. Optics Letters. 2006;31:2798-2800. DOI: 10.1364/ OL.31.002798

[74] Bocquillon E, Couteau C, Razavi M, Laflamme R, Weihs G. Coherence measures for heralded singlephoton sources. Physical Review A. 2009;79:035801. DOI: 10.1103/PhysRevA.79.035801

[75] Fortsch M, Furst JU, Wittmann C, Strekalov D, Aiello A, Chekhova MV, et al. A versatile source of single photons for quantum information processing. Nature Communications. 2013;4:1818. DOI: $10.1038 /$ ncomms 2838 
[76] Clark AS, Collins MJ, Judge AC, Mägi EC, Xiong C, Eggleton BJ. Raman scattering effects on correlated photon pair generation in chalcogenide. Optics Express. 2012;20:16807-16814. DOI: 10.1364/OE.20.016807

[77] Mosley PJ, Lundeen JS, Smith BJ, Wasylczyk P, U'Ren AB, Silberhorn C, et al. Heralded generation of ultrafast single photons in pure quantum states. Physical Review Letters. 2008;100:133601. DOI: 10.1103/ PhysRevLett.100.133601

[78] Rarity JG, Tapster PR, Jakeman E, Larchuk T, Campos RA, Teich MC, et al. Two-photon interference in a Mach-Zehnder interferometer. Physical Review Letters. 1990;65:1348. DOI: 10.1103/PhysRevLett.65.1348

[79] Clauser JF, Horne MA, Shimony A, Holt RA. Proposed experiment to test local hidden-variable theories. Physical Review Letters. 1969;23:880-884. DOI: 10.1103/PhysRevLett.23.880

[80] Collins D, Gisin N, Linden N, Massar S, Popescu S. Bell inequalities for arbitrarily high dimensional systems. Physical Review Letters. 2002;88:040404. DOI: 10.1103/ PhysRevLett.88.040404

[81] James DFV, Kwiat PG, Munro WJ, White AG. Measurement of qubits. Physical Review A. 2001;64:052312. DOI: 10.1103/PhysRevA.64.052312

[82] Blinov BB, Moehring DL, Duan LM, Monroe C. Observation of entanglement between a single trapped atom and a single photon. Nature. 2004;428:153157. DOI: $10.1038 /$ nature 02377

[83] Matsukevich DN, KA. Quantum state transfer between matter and light. Science. 2004;306:663-666. DOI: 10.1126/science. 1103346

[84] Togan E, Chu Y, Trifonov AS, Jiang L, Maze J, Childress L, et al.
Quantum entanglement between an optical photon and a solid-state spin qubit. Nature. 2010;466:730-734. DOI: 10.1038/nature09256

[85] Piro N, Rohde F, Schuck C, Almendros M, Huwer J, Ghosh J, et al. Heralded single-photon absorption by a single atom. Nature Physics. 2011;7:1720. DOI: $10.1038 /$ nphys 1805

[86] Clausen C, Usmani I, Bussieres F, Singouard N, Afzelius M, de Riedmatten $\mathrm{H}$, et al. Quantum storage of photonic entanglement in a crystal. Nature. 2011;469:508-511. DOI: 10.1038/ nature09662

[87] Kumar P. Quantum frequency conversion. Optics Letters. 1990;15:1476-1478. DOI: 10.1364/ OL.15.001476

[88] Tanzilli S, Tittel W, Halder M, Alibart O, Baldi P, Gisin N, et al. A photonic quantum information interface. Nature. 2005;437:116-120. DOI: $10.1038 /$ nature04009

[89] Takesue H. Single-photon frequency down-conversion experiment. Physical Review A. 2010;82:013833. DOI: 10.1103/PhysRevA.82.013833

[90] Curtz N, Thew R, Simon C, Gisin N, Zbinden $\mathrm{H}$. Coherent frequency downconversion interface for quantum repeaters. Optics Express. 2010;18:2209922104. DOI: 10.1364/OE.18.022099

[91] Zaske S, Lenhard A, Kebler CA, Kettler J, Hepp C, Arend C, et al. Visible-to-telecom quantum frequency conversion of light from a single quantum emitter. Physical Review Letters. 2012;109:147404. DOI: 10.1103/ PhysRevLett.109.147404

[92] Takesue H. Erasing distinguishability using quantum frequency up-conversion. Physical Review Letters. 2008;101:173901. DOI: 10.1103/PhysRevLett.101.173901 
[93] Rakher MT, Ma L, Slattery O, Tang X, Srinivasan K. Quantum transduction of telecommunications-band single photons from a quantum dot by frequency upconversion. Nature Photonics. 2010;4:786-791. DOI: 10.1038/nphoton.2010.221

[94] Guinness HJ, Raymer MG, McKinstrie CJ, Radic S. Quantum frequency translation of singlephoton states in a photonic crystal fiber. Physical Review Letters. 2010;105:093604. DOI: 10.1103/ PhysRevLett.105.093604

[95] Ates S, Agha I, Gulinatti A, Reach I, Rakher MT, Badolato A, et al. Two-photon interference using background-free quantum frequency conversion of single photons emitted by an InAs quantum dot. Physical Review Letters. 2012;109:147405. DOI: 10.1103/ PhysRevLett.109.147405

[96] Ikuta R, Kusaka Y, Kitano T, Kato H, Yamamoto T, Koashi M, et al. Wide-band quantum interface for visible-to-telecommunication wavelength conversion. Nature Communications. 2011;2:537. DOI: 10.1038/ncomms1544

[97] Guerrerio T, Martin A, Sanguinetti B, pelc JS, Langrock C, Fejer MM, et al. Nonlinear interaction between single photons. Physical Review Letters. 2014;113:173601. DOI: 10.1103/PhysRevLett.113.173601

[98] Zhou ZY, Li Y, Ding DS, Zhang W, Shi S, Shi BS, et al. Orbital angular momentum photonic quantum interface. Light: Science and Applications. 2016;5:e16019. DOI: 10.1038/lsa.2016.19

[99] Zhou ZY, Liu SL, Li Y, Ding DS, Zhang W, Shi S, et al. Orbital angular momentum-entanglement frequency transducer. Physical Review Letters. 2016;117:103601. DOI: 10.1103/ PhysRevLett.117.103601
[100] Zhou ZY, Liu SL, Liu SK, Li YH, Ding DS, Guo GC, et al. Superresolving phase measurement with shortwavelength NOON states by quantum frequency up-conversion. Physical Review Applied. 2017;7:064025. DOI: 10.1103/PhysRevApplied.7.064025

[101] Liu SL, Liu SK, Li YH, Shi S, Zhou ZY, Shi BS. Coherent frequency bridge between visible and telecommunications band for vortex light. Optics Express. 2017;25:2429024298. DOI: 10.1364/OE.25.024290

[102] Walker T, Miyanishi K, Ikuta R, Takahashi H, Kashanian SV, Tsujimoto Y, et al. Long-distance dingle photon transmission from a trapped ion via quantum frequency conversion. Physical Review Letters.

2018;120:203601. DOI: 10.1103/

PhysRevLett.120.203601

[103] Ikuta R, Kobayashi T, Kawakami T, Miki S, Yabuno M, Yamashita T, et al. Polarization insensitive frequency conversion for an atom-photon entanglement distribution via a telecom network. Nature Communications. 2018;9:1997. DOI: 10.1038/ s41467-018-04338-x

[104] Bock M, Eich P, Kucera S, Kreis M, Lenhard A, Becher C, et al. High-fidelity entanglement between a trapped ion and a telecom photon via quantum frequency conversion. Nature Communications. 2018;9:1998. DOI: 10.1038/s41467-018-04341-2

[105] Dréau A, Tchebotareva A, Mahdaoui AE, Bonato C, Hanson R. Quantum frequency conversion of single photons from a nitrogen-vacancy center in diamond to telecommunication wavelengths. Physical Review Applied. 2018;9:064031. DOI: 10.1103/

PhysRevApplied.9.064031

[106] Shentu GL, Pelc JS, Wang XD, Sun QC, Zheng MY, Fejer MM, et al. Ultralow noise up-conversion detector 
and spectrometer for the telecom band. Optics Express. 2013;21:13986-13991. DOI: 10.1364/OE.21.013986

[107] Liu SL, Zhou Q, Zhou ZY, Liu SK, Li Y, Li YH, et al. Multiplexing heralded single photons in orbital angular momentum space. Physical Review A. 2019;100:013833. DOI: 10.1103/PhysRevA.100.013833

[108] Migdall AL, Branning D, Castelletto S. Tailoring single-photon and multiphoton probabilities of a single-photon on-demand source. Physical Review A. 2002;66:053805. DOI: 10.1103/PhysRevA.66.053805

[109] Collins MJ, Xiong C, Rey IH, Vo TD, He J, Shahnia S, et al. Integrated spatial multiplexing of heralded single-photon sources. Nature Communications. 2013;4:2582. DOI: 10.1038/ncomms3582

[110] Kaneda F, Christensen BG, Wong JJ, Park HS, McCusker KT, Kwiat PG. Time-multiplexed heralded single-photon source. Optica. 2015;2:1010-1013. DOI: 10.1364/ OPTICA.2.001010

[111] Puigibert MG, Aguilar GH, Zhou Q, Marsili F, Shaw MD, Verma VB, et al. Heralded single photons based on spectral multiplexing and feedforward control. Physical Review Letters. 2017;119:083601. DOI: 10.1103/ PhysRevLett.119.083601

[112] Joshi C, Farsi A, Clemmen S, Ramelow S, Gaeta AL. Frequency multiplexing for quasi-deterministic heralded single-photon sources. Nature Communications. 2018;9:847. DOI: 10.1038/s41467-018-03254-4

[113] Nasr MB, Carrasco S, Saleh BEA, Sergienko AV, Teich MC, Torres JP, et al. Ultrabroadband biphotons generated via chirped quasi-phase-matched optical parametric down-conversion. Physical
Review Letters. 2008;100:183601. DOI:

10.1103/PhysRevLett.100.183601

[114] Kues M, Reimer C, Roztocki P, Cortés LR, Sciara S, Wetzel B, et al. On-chip generation of high-dimensional entangled quantum states and their coherent control. Nature. 2017;546:622. DOI: $10.1038 /$ nature22986

[115] Liu SL, Zhou ZY, Liu SK, Li YH, Li Y, Yang C, et al. Coherent manipulation of a three-dimensional maximally entangled state. Physical Review A. 2018;98:062316. DOI: 10.1103/PhysRevA.98.062316

[116] Kovlakov EV, Straupe SS, Kulik SP. Quantum state engineering with twisted photons via adaptive shaping of the pump beam. Physical Review A. 2018;98:060301. DOI: 10.1103/PhysRevA.98.060301

[117] Liu SL, Yang C, Xu ZH, Liu SK, Li Y, Li YH, et al. A high-dimensional quantum frequency converter. arXiv:1908.10569 [quant-ph]

[118] Wang JW, Paesani S, Dong YH, Santagati R, Skrzypczyk P, Salavrakos A, et al. Multidimensional quantum entanglement with largescale integrated optics. Science. 2018;360:285-291. DOI: 10.1126/science. aar7053

[119] Qiang X, Zhou X, Wang J, Wilkes CM, Loke T, O'Gara S, et al. Large-scale silicon quantum photonics implementing arbitrary two-qubit processing. Nature Photonics. 2018;12:534-539. DOI: 10.1038/ s41566-018-0236-y 
Chapter 4

\title{
Single-Photon Frequency Conversion for Quantum Interface
}

\author{
Yuanhua Li and Xianfeng Chen
}

\begin{abstract}
Single-photon frequency conversion for quantum interface plays an important role in quantum communications and networks, which is crucial for the realization of quantum memory, faithful entanglement swapping and quantum teleportation. In this chapter, we will present our recent experiments about single-photon frequency conversion based on quadratic nonlinear processes. Firstly, we demonstrated spectrum compression of broadband single photons at the telecom wavelength to the near-visible window, marking a critical step towards coherent photonic interface. Secondly, we demonstrated the nonlinear interaction between two chirped broadband single-photon-level coherent states, which may be utilized to achieve heralding entanglement at a distance. Finally, we theoretically introduced and experimentally demonstrated single-photon frequency conversion in the telecom band, enabling switching of single photons between dense wavelengthdivision multiplexing channels. Moreover, quantum entanglement between the photon pair is maintained after the frequency conversion. Our researches have realized three significant quantum interfaces via single-photon frequency conversion, which hold great promise for the development of quantum communications and networks.
\end{abstract}

Keywords: quantum interface, quantum network, single-photon frequency conversion, periodically poled lithium niobate waveguide, sum frequency generation, cascaded nonlinear process, spectrum compression, spontaneous down-conversion

\section{Introduction}

In recent years, nonlinear quantum optics has developed rapidly, such as quantum communication [1], quantum computation [2], quantum memory [3], quantum network [4], and so on. In order to realize these quantum applications, coherent quantum interface is a significant quantum device as it is capable of frequency and bandwidth in the telecom band is converted simultaneously. Quantum network is an important platform to study quantum communication, quantum computation, and quantum memory. Quantum network consists of many nodes and the quantum communication channels of the connected nodes, and the quantum communication channels of different connected nodes need to be connected by a quantum interface. Any node in a quantum network has the capability of quantum communication, quantum memory, quantum entanglement swapping, and generation of single photon sources. When the quantum channel of different nodes 
performs the conversion of quantum communication and quantum memory, a quantum interface is needed, which can simultaneously realize spectral compression and frequency conversion because the bandwidth and the center frequency of the single photon used in quantum communication and quantum memory are different. When two broadband photons of different nodes' quantum channels are connected by quantum entanglement swapping, the connected quantum interface can efficiently couple the two broadband photons and simultaneously realize the nonlinear frequency conversion of the two broadband photons. The nonlinear upconversion of two broadband photons in nonlinear crystals can be converted into a high frequency narrowband photon, which provides a basis for implementing different types of quantum interfaces.

Periodically polarized lithium niobate (PPLN) waveguides have higher secondorder nonlinear coefficients than other nonlinear crystals. PPLN waveguide not only transmits a wide spectrum but is also easily integrated and processed into PPLN waveguide chips. It also can preserve the quantum properties of photons. The nonlinear effect of PPLN waveguide chip can realize the up-conversion and down-conversion of nonlinear frequencies of different wavelength photons. Therefore, PPLN waveguide chip is ideal for implementing different types of quantum interfaces.

In our work, we utilized PPLN waveguide chip to realize several kinds of different functions of single-photon frequency conversion for coherent quantum interface. First, we have demonstrated the generated coherent quantum interface suitable for quantum communication at $1550 \mathrm{~nm}$ and quantum memory in the near-visible window. We exploit a positively chirped single-photon-level laser pulse and a negatively chirped classical laser pulse by sum frequency generation (SFG) process to compress the bandwidth of the positively chirped single-photon-level laser pulse in a PPLN waveguide chip_from 800 to $13.7 \mathrm{GHz}$ - which is approaching the bandwidth regime of some quantum memories. In the same time, one can flexibly convert the 1550 -nm telecom-band photons into the nearinfrared window [5]. Second, we have experimentally demonstrated the SFG between two broadband single-photon-level coherent states by using a highefficiency PPLN waveguide chip. The SFG efficiency of $1.06 \times 10^{-7}$ is realized, which provides potentially feasible quantum applications, such as faithful entanglement swapping without post-selection and nonlinear interaction between single photons with an integrated device at room temperature. What's more, longdistance quantum communication can be achieved by broadband single photons generated in a spontaneous parametric down conversion (SPDC) source [6]. Finally, we have realized the core quantum interface for fiber quantum networks. We report single-photon frequency conversion in a telecommunication band based on cascaded quadratic nonlinearity, i.e., SFG and difference frequency generation (DFG), in a PPLN waveguide. It shows that the frequency of single photons can be precisely converted to a DFG with continuous adjustability in a wide telecommunication band and their quantum characteristics are protected after the single-photon frequency conversion [7].

\section{Methodology and result}

\subsection{Spectral compression of single-photon-level laser pulse}

Photons at $1550 \mathrm{~nm}$ are critical to all quantum information tasks via an opticalfiber network. Nevertheless, the narrowband photons in the near-visible wavelength regime possess the most efficient quantum memories and an ability of being 
easily detected by a silicon avalanche photodiode (APD). Many theoretical schemes have been proposed to achieve the pulse compression or the frequency conversion. For instance, the 1550-nm photons can be converted into the near-infrared window through the nonlinear processes, such as the SFG. As theoretical schemes are diverse, they can be characterized by one common shortage, namely realizing only one operation. Therefore, it is highly expected that an optical technology is capable of simultaneously performing spectrum compression and frequency conversion in the telecom band.

In our experiment, spectral compression of single-photon-level laser pulse is generated by SFG between a positively chirped single-photon-level laser pulse and a negatively chirped classical laser pulse. As known, a laser pulse can be expressed as the frequency-dependent electric field $E(\nu)=U(\nu) e^{i \phi(\nu)}$, where $U(\nu)$ and $\phi(\nu)$ represent the amplitude information and phase information of the laser pulse, respectively. Obviously, we can obtain a chirp result when a transform-limited laser pulse is subject to a quadratic phase $\phi(\nu) \approx A\left(\nu-\nu_{0}\right)^{2}$, where $A$ is a constant and $\nu_{0}$ is the center frequency. In terms of $d \phi(\nu) / d \nu=2 \pi t$, one can obtain $d \phi(\nu) / d \nu=2 \pi t=2 A\left(\nu-\nu_{0}\right)$; thus, $\nu=\nu_{0}+\pi t / A$ is realized.

The negatively chirped classical laser pulse of frequency with $\nu_{0, P}$ increases linearly in time $t_{1}$, i.e., $\nu_{0, P}\left(t_{1}\right)=\nu_{0, P}+\pi t_{1} / A$, and the positively chirped singlephoton-level pulse frequency with $\nu_{0, Q}$ decreases linearly in time $t_{2}, \nu_{0, Q}\left(t_{2}\right)=$ $\nu_{0, Q}-\pi t_{2} / A$. Here, $A$ is the chirp rate, $\nu_{0, P}$ and $\nu_{0, Q}$ are the center frequency of these two laser pulse sources, and $\Delta t=t_{1}-t_{2}$ is a relative time delay between the negatively chirped classical laser pulse and positively chirped single-photon-level laser pulse. In our work, $\nu_{0, P}$ and $\nu_{0, Q}$ are equal as chirped laser pulse and negatively laser pulse are two replicas of the laser source. When the positively chirped singlephoton-level laser pulse and negatively classical laser pulse simultaneously reach the PPLN waveguide chip, a blue-shifted frequency component of negatively classical laser pulse $\left(\nu_{0, P}+\pi t_{1} / A\right)$ would match a red-shifted frequency component of positively chirped single-photon-level laser pulse $\left(\nu_{0, Q}-\pi t_{2} / A\right)$ with the relative time delay $\Delta t$, and all frequency components of these two laser pulses would sum to a new narrow frequency with $\nu_{0, S F G}(\Delta t)=\nu_{0, P}+\nu_{0, Q}+\pi \Delta t / A$. The expected (TH) intensity bandwidth (FWHM) of the SFG photon is given by the following equation [8]:

$$
\Delta \nu_{S F G}^{T H} \approx \frac{\ln 4}{A} \sqrt{\frac{1}{\Delta \nu_{P}^{2}}+\frac{1}{\Delta \nu_{Q}^{2}}},
$$

where $\Delta \nu_{P}$ and $\Delta \nu_{Q}$ are the bandwidths (FWHM). The FWHM bandwidth $\Delta \nu$ is limited by group velocity dispersion, and decreases linearly with the length of the PPLN waveguide, i.e., $\Delta \nu=\Delta \hat{\nu} / L$, where $\Delta \hat{\nu}=4200 \mathrm{GHz} \cdot \mathrm{cm}$ is the spectral acceptance of the waveguide. When the full FWHM bandwidths of positively chirped single-photon-level laser pulse and negatively chirped classical laser pulse are used in the waveguide, the maximum SFG efficiency is guaranteed.

A schematic of our experimental setup is shown in Figure 1. In our experiment, the mode-locked optical-fiber laser pulse source generates 500 -fs pulses at $1551.54 \mathrm{~nm}$ center wavelength, about $6.4 \mathrm{~nm}$ spectral bandwidth $(6.4 \mathrm{~nm}$ is the FWHM of the spectral intensity distribution), $59.98 \mathrm{MHz}$ repetition rate, and 45.2 MW average power. We split the laser pulse source into two replicas using a 50:50 beam splitter (BS), and then one of the two replicas of laser pulse is sent to a broadband fiber Bragg grating 1 (FBG1); at the same time, the other laser pulse is coupled into the FBG2. The parameters of FBG1 and FBG2 are exactly the same (1547 $\mathrm{nm}$ center wavelength, $39 \mathrm{~nm}$ FWHM bandwidth, and $5 \mathrm{~nm} / \mathrm{cm}$ chirp rate). 


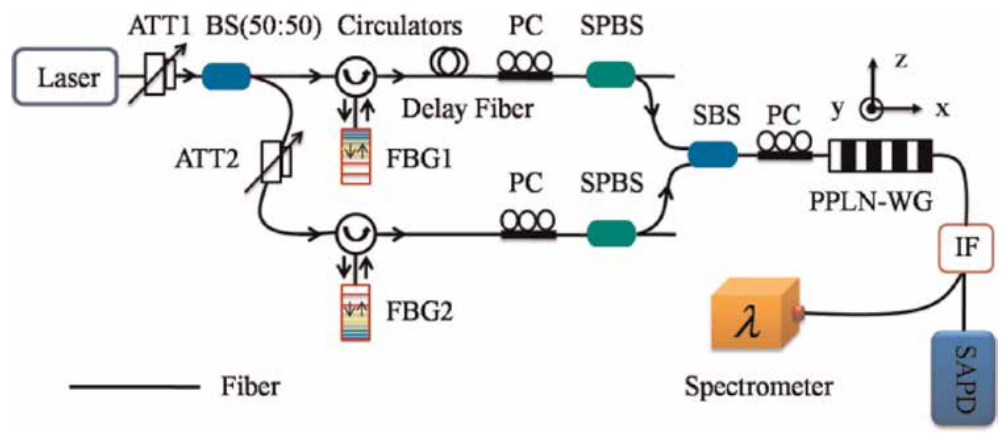

Figure 1.

Experimental set-up. ATT, variable optical attenuator; BS, beam splitter (50:50); Circulators, optical fiber circulators; PC, polarization controller; FBG, fiber Bragg grating; Delay Fiber, optical adjustable delay fiber; $S P B S$, single mode polarization beam splitter (single mode to polarization maintaining); SBS, single mode beam splitter (single mode to polarization maintaining); PPLN-WG, PPLN waveguide chip; IF, interference filter; $S A P D$, silicon APD [5].

It is known that FBG can be used for up-chirping and down-chirping, depending on the choice of the side from which the laser pulse is reflected. Thus, the two different chirp laser pulses after FBG1 and FBG2 are the same but with the opposite sign. It means that the positively chirped and negatively laser pulses have an equal and opposite chirp, $\pm A$. A positively chirped laser pulse is generated through FBG1 to introduce a linear chirp by group velocity dispersion. The other laser pulse is negative after a broadband FBG2. A variable optical attenuator (ATT1) is used to create a positively chirped single-photon-level laser pulse, and the other variable optical attenuator (ATT2) is used to control the energy of negatively chirped classical laser pulse for this experiment.

Then, the positively chirped single-photon-level laser pulse and the negatively chirped classical laser pulse are combined by a 1550/1550 nm 50:50 single-mode beam splitter (SBS) and couple into the z-cut PPLN waveguide chip through the optical-fiber pigtail. Two single-mode polarization beam splitters (SPBS (200:1)) and two polarization controllers (PCs) are used for controlling the positively chirped single-photon-level laser pulse and the negatively chirped classical laser pulse to the TM mode; it is known that the PPLN waveguide chip only supports Type-0 (ee $\rightarrow e$ ) phase matching in our work. A temperature controller (TC) is used for adjusting the PPLN waveguide chip's temperature to keep the QPM of the SFG process. The spectrally narrowed SFG photon pulse can be obtained in the PPLN waveguide chip, after interference filter (IF) with a nominal bandwidth of $20 \mathrm{~nm}$ (FWHM) centered around $780 \mathrm{~nm}$ (loss is about $1.2 \mathrm{~dB}$ ), and then coupled into an optical-fiber-coupled spectrometer. Finally, the SFG photons are detected by a SAPD, whose detection efficiency is up to $60 \%$ at $775 \mathrm{~nm}$ and dark count rate is $26 \mathrm{~Hz}$. In our experiment, a superconducting single photon detector (SSPD) is used to calibrate and monitor the counts of photons of positively chirped single-photon-level laser pulse, whose detection efficiency is up to $10 \%$ at $1551 \mathrm{~nm}$ and dark count rate is $600 \mathrm{~Hz}$. Using the IF, any residue of the positively or negatively chirped photons have to be filtered out from the SFG photons by a factor of $10^{-18}$.

The reverse-proton-exchange PPLN waveguide chip is 52-mm long and QPM to perform the SFG process $1551 \mathrm{~nm}+1551 \mathrm{~nm} \rightarrow 775.5 \mathrm{~nm}$. It is poled through the quasi-phase-matching period of $19.6 \mu \mathrm{m}$. Moreover, one has a total fiber-to-outputfacet throughput of approximately $-1.5 \mathrm{~dB}$ for the telecom band. The PPLN waveguide chip is antireflection coated to prevent interference fringes and enhance the system efficiency. 
We first obtain the spectrum of the positively chirped laser pulse by using an optical-fiber-coupled spectrometer and find a width $800 \pm 20 \mathrm{GHz}$ FWHM centered at $1551.54 \mathrm{~nm}$ (shown in red). The positively chirped laser pulse is then coupled through an optical fiber and superposed with the negatively chirped classical laser pulse $(790 \pm 20 \mathrm{GHz}$, centered at $1551.54 \mathrm{~nm})$ in the PPLN waveguide chip for SFG. The SFG photons, after IF, are sent to a single-mode optical fiber and coupled into the optical-fiber-coupled spectrometer. Here, the FWHM bandwidths of both negatively and positively chirped laser pulses are smaller than the spectral acceptance of the PPLN waveguide chip $(\Delta \nu=\Delta \hat{\nu} / L=807 \mathrm{GHz})$. Thus, the full FWHM bandwidths of positively and negatively chirped laser pulses are used in the PPLN waveguide chip, as expected. As shown in Figure 2(a), we measure significant spectral compression of the positively chirped laser pulse. The initial bandwidth of the positively chirped laser pulse is $800 \mathrm{GHz}$ centered at $1551.54 \mathrm{~nm}$ (shown in red). When the quadratic phase is applied and the two laser pulses are up-converted, the bandwidth of laser pulse generated reduces to $33 \pm 1 \mathrm{GHz}$ (FWHM) centered at $775.77 \mathrm{~nm}$ (shown in black), where the relative time delay $\Delta t=0$.

Taking the resolution of our spectrometer into account, $\Delta \nu_{R}=30 \pm 1 \mathrm{GHz}$ (FWHM), the actual bandwidth of the SFG photons after deconvolution is $\Delta \nu_{S F G}^{E X P}=\sqrt{\Delta \nu_{M}^{2}-\Delta \nu_{R}^{2}}=13.7 \pm 4.2 \mathrm{GHz}$ (FWHM). The spectra are given by normalized intensities and, for the up-converted case, correspond to the average of 10 consecutive scans of $15 \mathrm{~min}$ acquisition time. This result agrees closely with theory, $\Delta \nu_{S F G}^{T H}=9.8 \pm 0.7 \mathrm{GHz}$ (FWHM) from Eq. (1), using the expected chirp parameter $A=(-2.52 \pm 0.01) * 10^{8} f^{2}$ given by the geometry of our FBG. Therefore, a bandwidth compression ratio of 58:1 is achieved in the positively chirped laser pulse (Figure 2(b)).

The center wavelength of the SFG photons can be controlled by adjusting the relative delay $\Delta t$ between the input pulses at the PPLN waveguide chip. The SFG spectrum of the created laser pulse could be given by a function of the delay time, with the fitted center wavelengths shown in Figure 3. The experimental results show that the wavelength depends linearly on the delay time, as expected. The linear fit gives a slope of $-0.0247 \pm 0.001 \mathrm{~nm} / \mathrm{ps}$. In terms of the slope data, we measure the negatively chirp parameter of $A=(-2.55 \pm 0.01) * 10^{8} f^{2}$, in good agreement with the chirp parameter $A=(-2.52 \pm 0.01) * 10^{8} f s^{2}$ of the FBG1. It is also shown that the bandwidth compression ratio independent of the optical relative delay $\Delta t$, which agrees closely with the theoretical result from the above Eq. (1).
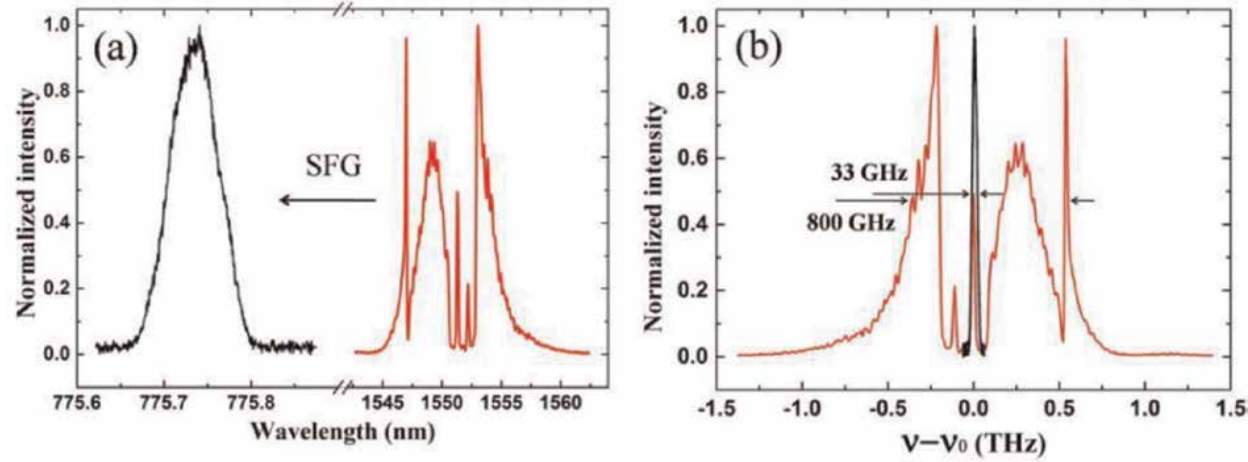

Figure 2.

The positively chirped pulse spectrum and up-converted laser pulse spectrum (a) and relative frequency (b) [5]. 

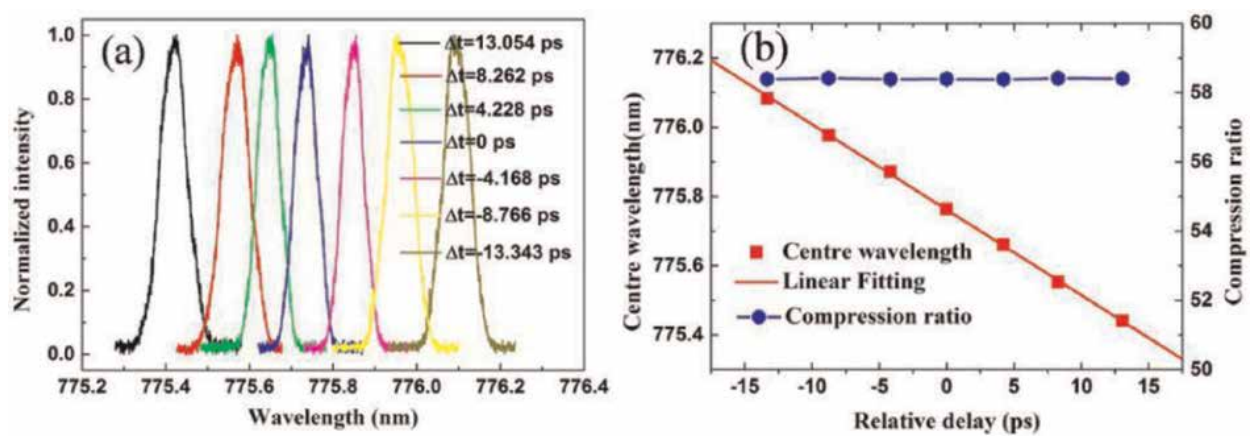

Figure 3.

The SFG spectrum of the created laser pulse (a), central wavelength and compression ratio of the output pulses versus the optical relative delay (b). Error bars are smaller than the data points [5].

In our experiment, it is verified that any SFG photons detected by the SAPD is the result of the SFG process, and not a SHG of the positively or negatively chirped photons. Moreover, any residue of photons the positively or negatively chirped laser pulse has to be filtered out from SFG photons by a factor of $10^{-18}$. Once the number of positively chirped laser pulse is controlled to single-photon level, the detected SHG counts from the positively chirped laser pulse drop to its dark counts $(3.5 \mathrm{~Hz})$. When the input power of the negatively chirped laser pulse is less than $0.6 \mathrm{nW}$, the SHG photons are also equal to the dark counts. The efficiency of SFG is then given by $\eta_{S F G}=P_{S F G} / \beta N$, where $P_{S F G}$ is the number of SFG photons per second, $\beta$ is the repetition rate of seed laser, and $N$ is average of photons per second of positively chirped laser pulse.

As shown in Figure 4, the power of the negatively chirped laser pulse is keeping at $0.6 \mathrm{nW}$. The SFG photons and SFG efficiencies are measured. The average of photons per second $\left(N_{1}=0.933\right.$ and $\left.N_{2}=0.302\right)$ of positively chirped laser pulse can be obtained with the ATT2. By adjusting the relative delay $\Delta t$, SFG photons and $\mathrm{SFG}$ efficiencies of different situations are measured (like Figure 3(b)). At the same time, it is found that the overall conversion efficiency of SFG varies with the relative delay $\Delta t$. When the relative time delay $\Delta t=0$, the maximum SFG efficiency is $7.82 \times 10^{-6}$ with the average of photons of positively chirped laser pulse (0.933), where the dark counts $(3.5 \mathrm{~Hz})$ are subtracted. Here, the total losses have

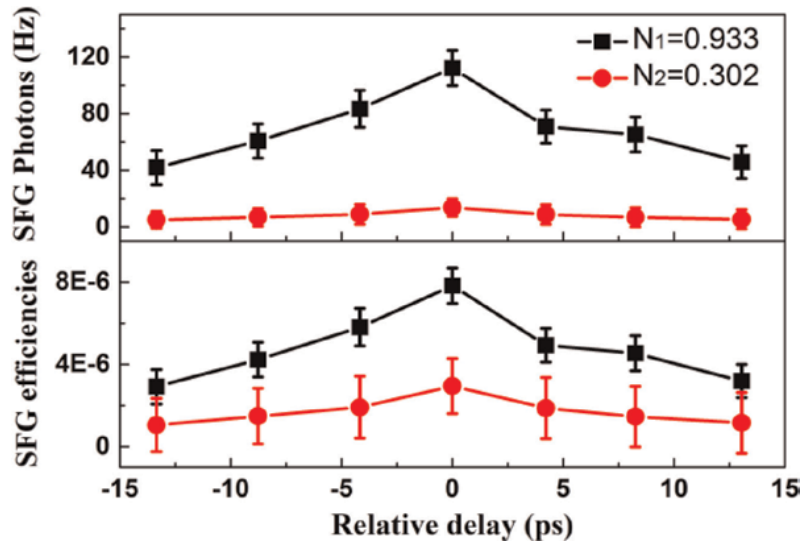

Figure 4 .

SFG photons (top) and SFG efficiencies (bottom). The abscissa is a variable optical relative delay between the negatively and positively chirped laser pulses at the PPLN waveguide chip [5]. 
been considered, such as the coupling loss of $0.7 \mathrm{~dB}$, reflection loss of $1.2 \mathrm{~dB}$, total fiber-to-output-facet loss of $1.5 \mathrm{~dB}$, and detection efficiency of $60 \%$ (see Figure 4).

The lower SFG signal for single photons required longer times than for the intense photons states. Thus, all the data are measured within a day to reduce the effects of drift. The results show that the SFG efficiency increases with increasing the average of photons of the positively chirped pulse (see Figure 4).

It is found that the SFG efficiency decreases with reducing the photons of negatively chirped laser pulse, and the efficiency of SFG also increases with increasing input power of the positively and negatively chirped laser pulse. Next, we obtain the efficiency of SFG in two ways: one, by controlling the photons of the negatively chirped laser pulse with the ATT1 and ATT2; the other by increasing the power of positively and negatively chirped laser pulse with the ATT1 and ATT2.

Figure 5 depicts the results of these two measurements. The SFG efficiencies, SFG photons, the power of produced harmonics, and error bars of them are accounted, where the dark counts $(3.5 \mathrm{~Hz})$ are subtracted.

As shown in Figure 5(a), when the negatively chirped laser pulse (10 photons per pulse) and the positively chirped single-photon-level laser pulse ( 0.933 photons per pulse) are simultaneously sent to the PPLN waveguide chip, the maximum SFG efficiency of $4.58 \times 10^{-7}$ is obtained, where the relative time delay $\Delta t=0$. In

Figure 5(b), we use the ATT1 and ATT2 to keep the input power of positively and negatively chirped laser pulse at 203.1 and $202.8 \mu \mathrm{W}$, respectively. The power of produced harmonics $E_{i}(i=0,1,2)$ can be measured, where $E_{0}$ is the total power of SFG and SHG of the positively and negatively chirped laser pulse, $E_{1}$ is the power of SHG of the positively chirped laser pulse, and $E_{2}$ is the power of SHG of the negatively chirped laser pulse. When the relative time delay $\Delta t=0$, the power of SHG generated is $E_{0}=21.62 \mu \mathrm{W}$, which is obtained from SHG of the positively chirped laser pulse $\left(E_{1}=0.28 \mu W\right)$, SHG of the negatively chirped laser pulse $\left(E_{2}=0.01 \mu \mathrm{W}\right)$, and SFG photons $\left(E_{S F G}=E_{0}-E_{1}-E_{2}=21.33 \mu W\right)$. In the case, the maximum SFG efficiency of $20 \%$ is obtained, where the relative time delay $\Delta t=0$. Here, the total losses have been taken into account. We also find that the rate of SFG photons generated is 73 times of the rate of SHG photons generated of these two independent laser pulses.

In our scheme, although both the negatively and positively chirped laser pulses are at the same center wavelength, the power of SHG from each is kept below the power of SFG (see Figure 5(b)). At the same time, the SHG photons from the two independent laser pulses are also lower than the SFG photons when the bandwidth of positively chirped single-photon-level laser pulse is compressed
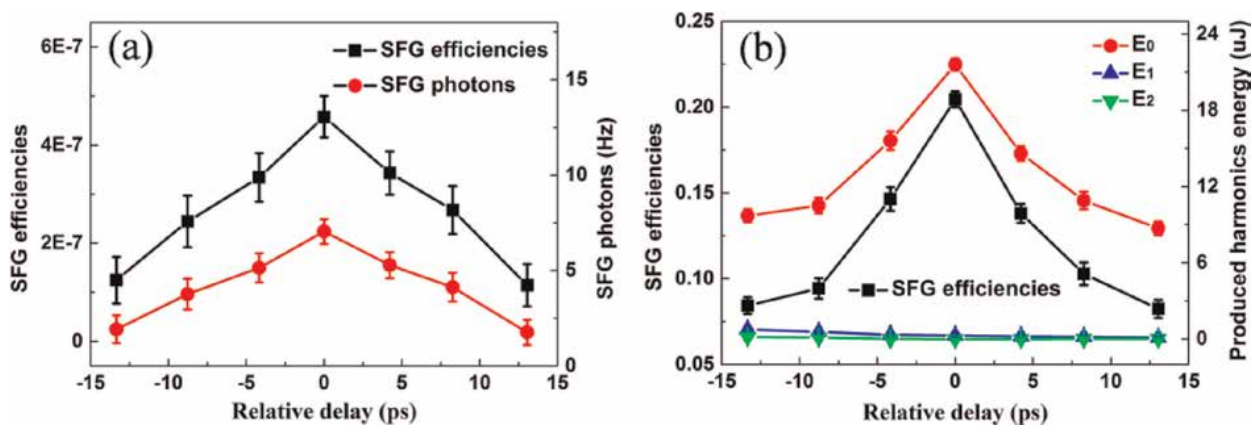

Figure 5.

SFG photons and SFG efficiencies (a), SFG efficiencies and the energy of generated SHG photons (b). The abscissa is a variable optical relative time delay between the negatively and positively chirped laser pulses at the PPLN waveguide chip [5]. 
(see Figures 4 and 5(a)). We can also confirm that by controlling the power of the negatively chirped laser pulse, considering a positively chirped single-photon-level laser pulse ( 0.933 photons per pulse). Figure 6 depicts the results of experimental and theoretical SFG and SHG.

Once the input power of the negatively chirped laser pulse is more than $0.6 \mathrm{nW}$, the up-converted photons generated consists of SHG photons of the negatively chirped laser pulse, and SFG photons of the negatively chirped laser pulse and the positively chirped single-photon-level laser pulse. In our experiment, up-converted photons cannot be filtered out separately; thus, the SFG photons $P_{S F G}$ cannot be measured. Here, we first send the negatively chirped laser pulse to the PPLN waveguide chip alone, and the SHG photons $P_{1}$ of the negatively chirped laser pulse can be measured. If we simultaneously couple the negatively chirped laser pulse and positively chirped single-photon-level laser pulse together into the PPLN waveguide chip, the photons $P_{0}$ of SFG and SHG can be obtained. The SFG photons generated are obtained with the equation $P_{S F G}=P_{0}-P_{1}$. When the relative time delay $\Delta t=0$, the SHG and SFG photons of different situations are obtained by controlling the power of the negatively chirped laser pulse (see Figure 6(a)). When the power of negatively chirped laser pulse is less than $4.8 \mathrm{nW}$, we find that SHG photons generated are lower than the SFG photons generated. When $P_{S F G}=P_{1}$, the SFG efficiency of $3.12 \times 10^{-5}$ is obtained. The measured results show that the number of SHG photons will be more than the number of SFG photons when the power of negatively chirped laser pulse is more than $4.8 \mathrm{nW}$.

Next, we carry out the theoretical analysis for SHG and SFG photons. As shown in Figure 6(b), the spectrum of the negatively chirped laser pulse is measured. It is found that the intensity of the negatively chirped laser pulse at $1551.54 \mathrm{~nm}$ is very close to zero. When the negatively chirped laser pulse is sent to the PPLN waveguide alone, the number of SHG photons generated is very low. Here, we assume that the power of the negatively chirped laser pulse which can be converted into SHG photons is $E_{N_{2}}$ (black area), thus, the SHG photons $P_{1} \propto\left|E_{N_{2}}\right|^{2}$. However, when the negatively and positively chirped laser pulses are simultaneously couple together into the PPLN waveguide chip, photons in all spectrum of the negatively chirped laser pulse can be used to produce the SFG photons. The full power of the negatively chirped laser pulse is $E_{N_{1}}$, as shown in Figure 6(b). Thus, the SFG photons $P_{S F G} \propto E_{N_{1}} E_{S}$, where $E_{S}$ is the power of the positively chirped singlephoton-level laser pulse. When $P_{S F G} / P_{1} \geq 1$, the number of SFG photons generated is more than the number of SHG photons. In the case, we obtain
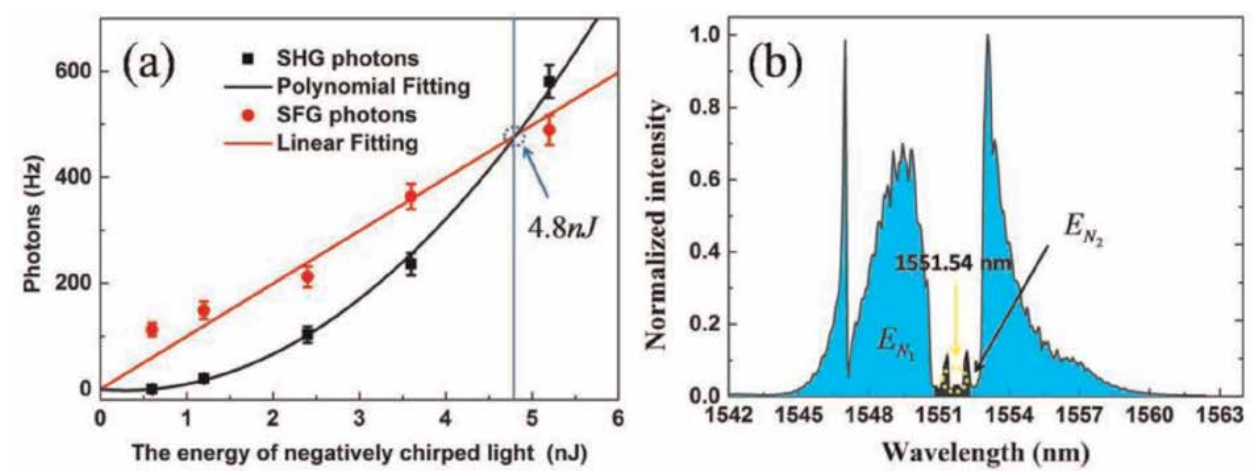

Figure 6.

SHG photons and SFG photons (a), the negatively chirped laser pulse spectrum (b). The dark counts (3.5 Hz) are subtracted, and the error bars are accounted [5]. 


$$
\frac{E_{N_{1}} E_{S}}{\left|E_{N_{2}}\right|^{2}} \geq 1 \text {. }
$$

In terms of $E_{N_{1}}=q E_{N_{2}}$, one can obtain $E_{N_{1}} \leq q^{2} E_{S}$, where $q$ is proportional coefficient. In our experiment, one has $E_{S}=7.3 \times 10^{-3} \mathrm{~nJ}$ and $q=26.8$; thus, $E_{N_{1}} \leq 5.2 \mathrm{~nJ}$ is realized. This result agrees closely with experiment, as shown in Figure 6(a).

In order to reduce the number of SHG photons to the dark counts $(3.5 \mathrm{~Hz})$, the power of the negatively chirped laser pulse must be very low. Thus, the SFG efficiency is limited. The way to improve SFG efficiency is to filter out the photons of wavelengths centered at $1551.54 \mathrm{~nm}$ of the negatively chirped laser pulse.

Furthermore, our results may provide potential application in standard decoystate quantum key distribution. By considering a fiber attenuation of $0.2 \mathrm{~dB} / \mathrm{km}$, the coupling loss of $0.7 \mathrm{~dB}$, the dark counts of $3.5 \mathrm{~Hz}$, total fiber-to-output-facet loss of $1.5 \mathrm{~dB}$, reflection loss of $1.2 \mathrm{~dB}$, detection efficiency of $60 \%$, and laser pulse with a $59.98 \mathrm{MHz}$ repetition rate, the SFG efficiency of $7.82 \times 10^{-6}$ will achieve a rate of about $8 \mathrm{bits} / \mathrm{h}$ on a distance of $20 \mathrm{~km}$.

\subsection{Nonlinear interaction between broadband single-photon-level coherent states}

It has been demonstrated that parametric interactions hold numerous advanced applications in quantum communication, but strong optical fields are usually used to preserve quantum property [9]. Nonlinear interactions between single photons have been experimentally measured, such as spontaneous down-conversion [10] and cross-phase modulation [11]. Here, we take the next step and report, a SFG between two broadband single-photon-level coherent states. In our experiment, the SFG efficiency of $1.06 \times 10^{-7}$ is realized, which provides potentially feasible quantum applications, such as faithful entanglement swapping without post-selection and nonlinear interaction between single photons with an integrated device at room temperature. What's more, long-distance quantum communication can be achieved by broadband single photons generated in a spontaneous parametric down conversion (SPDC) source.

In our experiment, a mode-locked optical fiber laser generates 500 -fs pulses at $1551 \mathrm{~nm}$ with a repetition of $59.98 \mathrm{MHz}$ and is used to generate the two chirped broadband single-photon-level coherent states after FBG1 and FBG2. These two chirped broadband coherent states are combined via a 50:50 SBS and sent to a PPLN waveguide chip. The PPLN waveguide chip length is $52 \mathrm{~mm}$, and its total losses are $2.2 \mathrm{~dB}$ including a coupling loss of $0.7 \mathrm{~dB}$ and a total fiber-to-output-facet loss of $1.5 \mathrm{~dB}$. The unconverted photons are deterministically separated from the SFG photons after IF, and the SFG photons are coupled into a silicon APD.

A schematic of the experimental setup is shown in Figure 7. The mode-locked optical fiber laser can generate two copies of the pulses with equal energy with a 50:50 BS. These two copies of the pulses are used to produce two broadband singlephoton-level coherent states with ATT1 and ATT2. One of the two copies of the laser pulses is couple into the FBG1, and the other laser pulse is coupled into the FBG2. Thus, the spectrums of two copies of the photons after FBG1 and FBG2 are the same but with the opposite sign. In our experiment, the positively chirped single-photon-level coherent state is generated with the FBG2, and the other single-photon-level coherent state is the negatively chirped coherent state after the FBG1. 


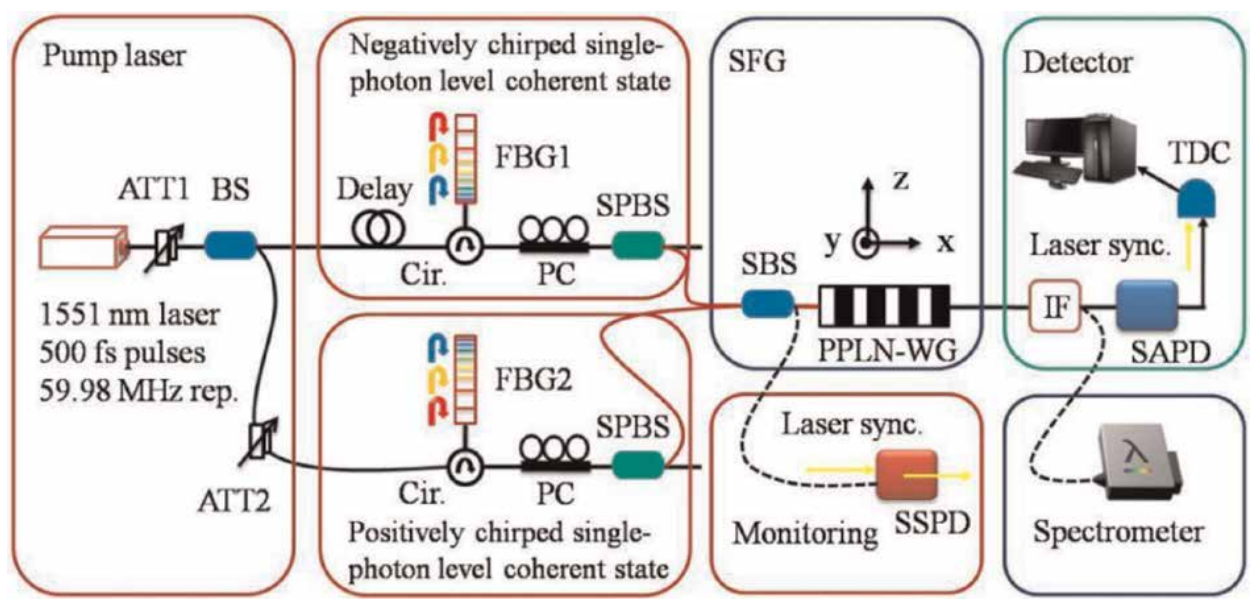

Figure 7.

Experimental set-up. ATT, variable optical attenuator; BS, beam splitter (50:50); Circulators, optical fiber circulators; PC, polarization controller; FBG, fiber Bragg grating; Delay Fiber, optical adjustable delay fiber; $S P B S$, single mode polarization beam splitter (single mode to polarization maintaining); SBS, single mode beam splitter (single mode to polarization maintaining); PPLN-WG, PPLN waveguide chip; IF, interference filter; $S A P D$, silicon $A P D$ [6].

The FBG can be used to produce the up-chirping and down-chirping, depending on the choice of the side from which the laser pulse is reflected. As the two copies of the laser pulses are from the same seed laser, they have the same initially center frequency $\omega_{0}$. When two copies of the laser pulses with instantaneous frequencies described as $\omega_{1}(t)=\omega_{0}+A t$ and $\omega_{2}(t)=\omega_{0}-A t$ (where $A$ is the linear chirp parameter) undergo SFG, the frequency of the laser pulse generated is constant $\left(\omega_{1}(t)+\omega_{2}(t)=2 \omega_{0}\right)$; thus, the high frequency long narrowband laser pulse can be realized.

Subsequently, the positively and negatively chirped coherent states are sent to the PPLN waveguide chip by the fiber pigtail. The PPLN waveguide chip is a reverse-proton-exchange waveguide that is QPM to perform the SFG process $1551 \mathrm{~nm}+1551 \mathrm{~nm} \rightarrow 775.5 \mathrm{~nm}$. The PPLN waveguide chip has a QPM period of $19.6 \mu \mathrm{m}$. Two SPBS (200:1) and two PCs are used for controlling the positively and negatively chirped single-photon-level coherent states to the TM mode. A SSPD is used to calibrate and monitor the counts of the positively and negatively chirped single-photon-level photons, whose detection efficiency is up to $10 \%$ at $1551 \mathrm{~nm}$ and dark count rate of $600 \mathrm{~Hz}$. A stable TC is used to keep at $27^{\circ}$ to maintain the QPM condition of the SFG process. The long narrowed SFG photons of higher frequency are generated after the IF, with $20 \mathrm{~nm}$ FWHM bandwidth and $780 \mathrm{~nm}$ center wavelength (about $1.2 \mathrm{~dB}$ loss). Finally, the SFG photons are detected with a SAPD. The SFG photons and the laser clock signal are recorded using a TDC.

We first measure the spectrums of the negatively chirped laser pulse $(800 \pm 20$ GHz spectral FWHM bandwidth, and $1551.56 \mathrm{~nm}$ center wavelength) and the positively chirped laser pulse ( $790 \pm 20 \mathrm{GHz}$ FWHM, and $1551.56 \mathrm{~nm}$ center wavelength) by using a spectrometer, which are shown in Figure 8(a). The pump light (the negatively chirped laser pulse) and the signal light (the positively chirped laser pulse) are simultaneously sent to the PPLN waveguide chip. By using an IF, the generated SFG photons are sent into the spectrometer.

In our experiment, when the classical pump and signal laser pulses are coupled into the PPLN waveguide chip, the up-converted photons consist of SHG of pump light, SHG of signal light, and SFG of pump light and signal light. In our experiment, up-converted photons cannot be filtered out separately; thus, the SFG 

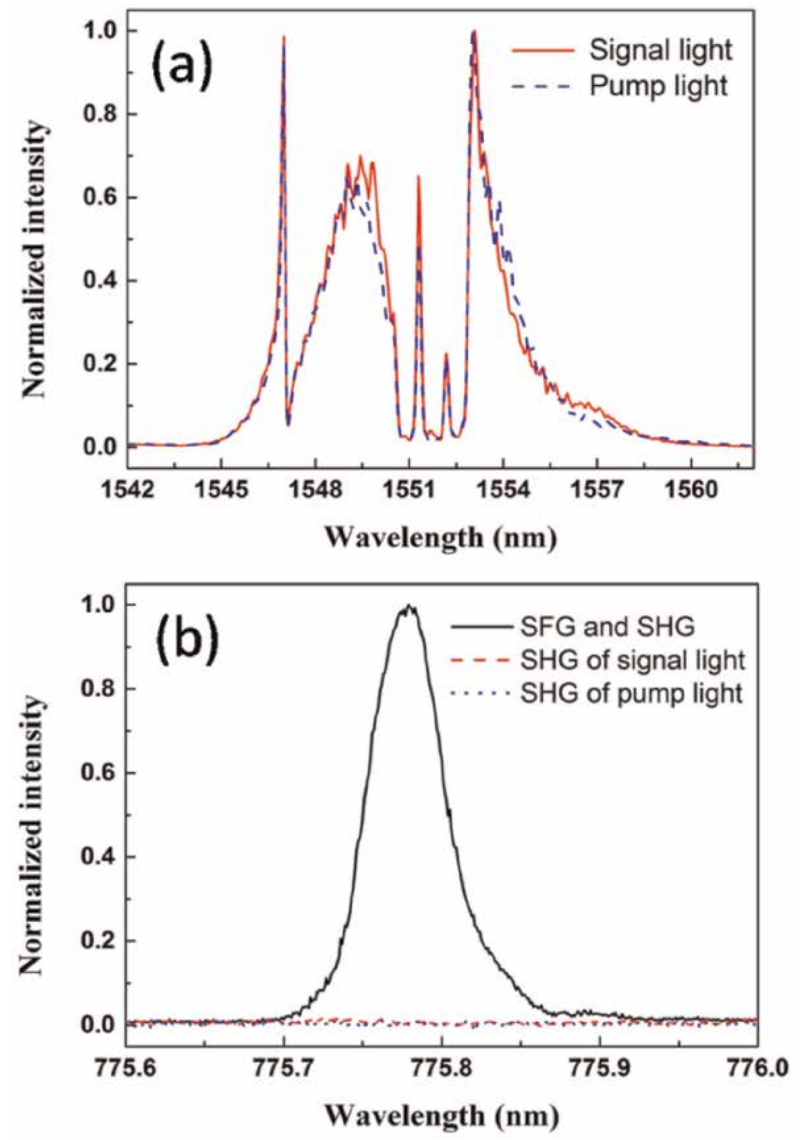

Figure 8.

Pump and signal light spectrums (a), and the spectrums of up-converted light (SFG and SHG), SHG of signal light, SHG of pump light (b) [6].

photons $N_{S F G}$ cannot be measured. First, the pump light is sent to the PPLN waveguide chip alone, and the number of SHG photons $N_{1}$ can be obtained. Similarly, the number of SHG photons $N_{2}$ of signal light is obtained when only the signal light is sent to the PPLN waveguide chip alone. When we simultaneously couple the pump and signal laser pulses together into the PPLN waveguide chip, we can obtain the number of photons $N_{0}$ of SFG and SHG. Therefore, the number of SFG photons is obtained with the equation $N_{S F G}=N_{0}-N_{1}-N_{2}$. For the SFG photons to be measured distinctly, any residue of pump and signal light has to be filtered out from upconversion photons in this work. Here, the efficiency of SFG is given by

$\eta_{S F G}=N_{S F G} / N_{A}$, where $N_{A}$ is the number of photons per second of the signal light.

For instance, the input power of the signal and pump light is keeping at 200.2 and $200.4 \mu \mathrm{W}$, respectively. When they are simultaneously sent to the PPLN waveguide chip, the FWHM bandwidth and power of the created up-conversion light can be measured. If pump light (or signal light) is sent to the PPLN waveguide chip alone, the bandwidth and power of SHG of pump light (or signal light) can be obtained. When the PPLN waveguide chip's temperature is kept at $27^{\circ}$, as shown in Figure 8(b), the FWHM bandwidth of the created up-conversion light is about $0.07 \mathrm{~nm}$, centered at $775.78 \mathrm{~nm}$. In this case, the power of up-conversion light is $20.9 \mu \mathrm{W}$ (involving the SFG and SHG), which is obtained from SHG of signal light $(0.25 \mu \mathrm{W})$, SHG of pump light $(0.01 \mu \mathrm{J})$, and SFG of pump light and signal light $(20.54 \mu \mathrm{W})$. The maximum SFG efficiency of $5 \%$ is obtained, which is used for 
estimating the efficiency of SFG between two single-photon-level states in our work. As shown in Figure 9, the efficiency of SFG depends on the PPLN waveguide chip's temperature. Correcting for all of these losses, the intrinsic device maximum SFG efficiency of $20 \%$ is obtained, as expected.

When the number of photons per pulse of pump light and signal light is attenuated to 5.13 and 5.64, respectively, the detected count of SHG of signal light (or pump light) drop to $3.8 \mathrm{~Hz}$ (dark count). It can be shown that any photons detected by the SPAD are the SFG photons when the signal and pump light are attenuated to single-photon-per-pulse simultaneously. Therefore, photons of SHG of pump light and signal light are not considered in our scheme.

In our experiment, the number of photons per pulse (equal for pump and signal) are obtained with ATT1 and ATT2, as they can be detected and calibrated by the SSPD. According to our experimental results, the SFG efficiency and SFG photons are shown in Figure 10. Here, dark counts of $3.8 \mathrm{~Hz}$ and the total losses of about $5.6 \mathrm{~dB}$ have been taken into account.

The overall conversion efficiency of SFG is given by $\eta_{S F G}^{\prime}=\xi(\lambda) h c \Delta \nu L^{2} / \lambda t b p$, where $\xi(\lambda)$ is the measured up-conversion efficiency of the PPLN waveguide chip, $\lambda$ is the center wavelength of pump light, tbp is the time-bandwidth product, $\Delta \nu$ is the bandwidth of pump light, and $L$ is the length of the PPLN waveguide chip. Consider that $\xi(\lambda)=5 \% /\left(W \cdot \mathrm{cm}^{2}\right)$ and $t b p=0.4$ in our experiment, thus the expected SFG efficiency is $\eta_{S F G}^{\prime} \approx 1 \times 10^{-7}$. We measure the efficiency of $\eta_{S F G}=(1.06 \pm 0.23) \times 10^{-7}$. It is shown that the SFG efficiency is high enough to provide efficient, yet simpler solutions to linear optics based protocols for the heralded creation of maximally entangled pairs or for the implementation of deviceindependent quantum key distribution.

For SFG between two single photons, our efficiency of SFG is about eight times of the efficiency of SFG given by Sangouard et al. [12]. When the single-photonlevel pump and signal light are not chirped in our experiment, the SFG photons are zero. This is because the intensity of pump light (or signal light) at $1551.56 \mathrm{~nm}$ is very low (see Figure 8(a)). However, when the negatively and positively chirped pulses are simultaneously coupled into the PPLN waveguide chip, photons in all spectrums of the negatively and positively chirped light are used to create the SFG photons. Therefore, we can improve the efficiency of SFG between single photons by using the chirped technology.

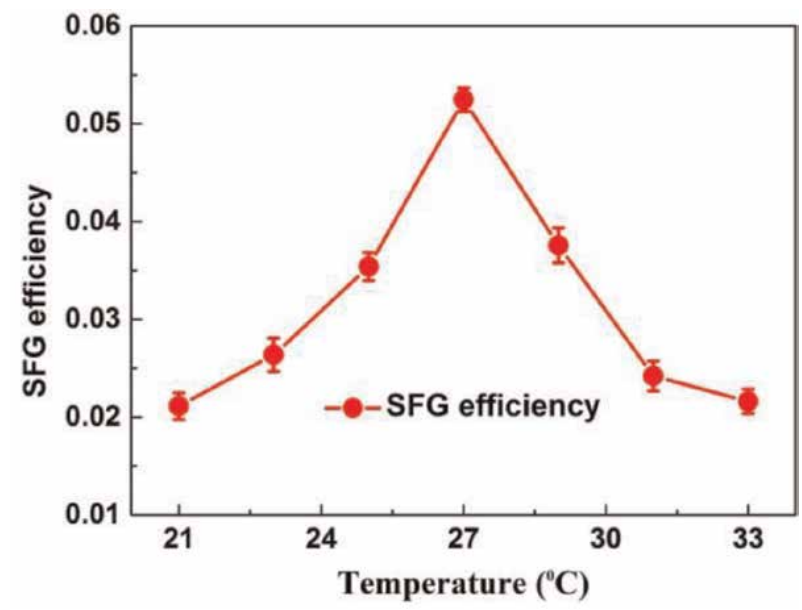

Figure 9.

SFG efficiency. The efficiency of SFG can be controlled by keeping the PPLN-WG chip's temperature [6]. 


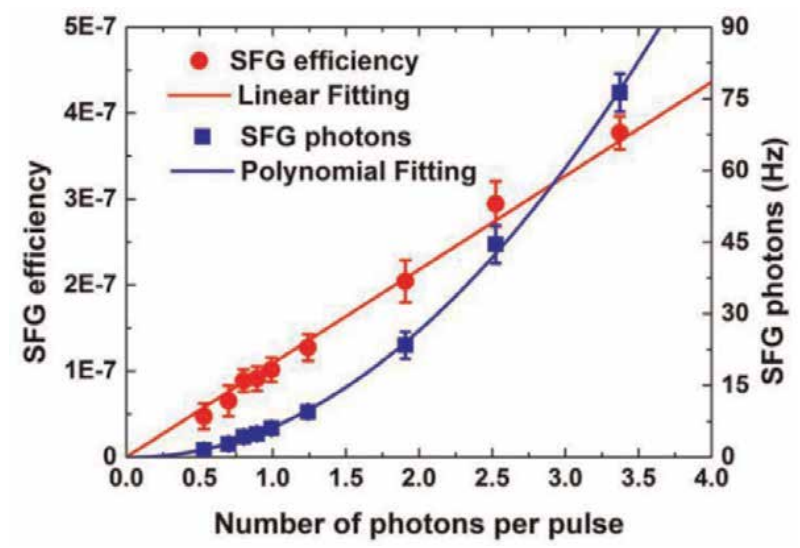

Figure 10.

SFG efficiency and SFG photons [6].

\subsection{Single-photon frequency conversion via cascaded quadratic nonlinear processes}

In quantum networks, many nodes are needed, which are used to perform quantum information memory/processing tasks. Each node has the ability to generate quantum states and perform quantum Bell-state measurements. All nodes are connected by using optical fibers. Frequency conversion has important applications in fiber quantum networks, and frequency converters are set to light. At the intermediate node of the fiber quantum network, the information transmission mode of the corresponding wavelength is obtained, and the specific wavelength signal carrying the information is transferred to the other wavelength. The development of this technology has greatly improved the transmission capacity of the optical network and the flexibility of the entire network.

In addition to the frequency conversion between the communication band and the visible band, single-photon frequency conversion between communication bands, like classical fiber networks, has important applications in large-scale quantum networks, as well as between independent sources of different users. Quantum cryptic transmission or quantum key distribution also requires different wavelengths to be converted to coincidence before interference. Therefore, the single photon frequency conversion interface between communication bands is critical in quantum communication.

However, there are not many experiments to achieve single-photon frequency conversion in the communication band. There are only two known solutions and each of these experimental solutions has imperfections. The first solution is to achieve high-precision frequency conversion by optical single sideband modulator (OSSB). This method can eliminate the frequency distinguishability between different single photons, but the frequency conversion range that this scheme can achieve covers only dozens. Gigahertz does not meet the requirements of communication networks. The second scheme is consistent with the four-wave mixing principle of the classical light in the previous section. The third-order nonlinearity of the optical fiber is used to realize the frequency conversion of the communication band, but we know that the third-order nonlinear coefficient is much smaller than 2. The order nonlinear coefficient, thus one must use $750 \mathrm{~m}$ long fiber, which is very unfavorable for device integration.

In order to make up for the shortcomings of the above two schemes, we use the high second-order nonlinear coefficients of the PPLN waveguide and the wide-band 
type-0 quasi-phase matching to realize the single-photon frequency conversion based on the cascaded second-order nonlinear process. In the experiment, we realized the precise frequency conversion between DWDM channels through the cascading process of SFG + DFG. At the same time, we also proved that the quantum characteristics of single photons remain in this process. Our experiment is very suitable for the construction of quantum networks.

The nonlinear process of frequency conversion of a single photon can be described by the following effective Hamiltonian [13]:

$$
\hat{H}=i \hbar\left(\chi_{1} E_{P 1} \hat{\alpha}_{s} \hat{\alpha}_{m}^{\dagger}+\chi_{2} E_{P 2} \hat{\alpha}_{m} \hat{\alpha}_{t}^{\dagger}-\text { H.c. }\right) \text {, }
$$

where $\hat{\alpha}_{i}$ a is the annihilation operator for the wave at frequency $\omega_{i},(i=s ; m ; t$ is signal, mediate, and target photons, respectively). $\chi_{1,2}$ are coupling constants that are proportional to the second-order susceptibility $\chi^{(2)}$ of the PPLN waveguide chip, $E_{P 1}$ and $E_{P 2}$ are the electric field amplitudes of pump lasers, and H.c. is a Hermitian conjugate. The conversion efficiency $\eta_{c}$ can be obtained by using the Heisenberg equation of motion, which is given by the following equation:

$$
\eta_{c}(L)=\frac{\eta_{1} \eta_{2} P_{P 1} P_{P 2}\left|\cos \left[2\left(\varphi_{1}-\varphi_{2}\right)\right]\right|}{\left(\eta_{1} P_{P 1}+\eta_{2} P_{P 2}\right)} \times\left\{1-\cos \left[\eta_{1} P_{P 1}+\eta_{2} P_{P 2}\right]^{(1 / 2)} L\right\}^{2},
$$

where $\varphi_{1}$ and $\varphi_{2}$ are the phases of the two pumps, respectively. $\eta_{1}$ and $\eta_{2}$ are the efficiencies of the normalized power, and $\eta_{1} \approx \eta_{2}=1.1 / \mathrm{W} \mathrm{cm}^{2}$. The efficiency of single-photon frequency conversion is $100 \%$ if $P_{P 1}=P_{P 2}=\pi^{2} /\left(2 \eta_{1} L^{2}\right)$.

It is very challenging for realizing simultaneous phase matching in our work. However, this problem can be easily solved using QPM. In the experiment, we realized the broadband single photon frequency conversion of the communication band by the type- 0 cascading SFG/DFG process. A 5-cm-long PPLN waveguide chip is used, and its poling period is $19.0 \mu \mathrm{m}$. In addition, the cascaded $\chi^{(2)}: \chi^{(2)}$ processes give rise to a large effective third nonlinearity typically $10^{4}-10^{5}$ times larger than a pure $\chi^{(3)}$ process, which manifests an advantage over its counterpart of FWM, e.g., in fibers.

The experimental setup of the single-photon frequency conversion is shown in Figure 11(a). The center wavelengths of the two auxiliary pumps P1 and P2 are $1547.72 \mathrm{~nm}$ (CH37) and $1544.53 \mathrm{~nm}$ (CH41), respectively. Both narrow-band continuous lasers increase power through an erbium-doped fiber amplifier (EDFA). A set of DWDMs placed behind the EDFA, using $150 \mathrm{~dB}$ of isolation to filter out the noise generated during the EDFA amplification process. Then, we use another set of DWDMs to combine the three signals of signal single photon, P1 and P2 into one beam and couple into the waveguide. The combined light undergoes a cascaded nonlinear process SFG + DFG in the PPLN waveguide, converting the signal photons into target photons. At the output of the waveguide, we use a third set of DWDMs to pick out the target photons, while using $180 \mathrm{~dB}$ of isolation to filter out the noise generated during P1, P2, and conversion. In our experimental scheme, frequency-adjustable single-photon frequency conversion can be achieved by adjusting the wavelength of the auxiliary pump light.

For the performance of the converter, we tested the photon pair prepared by SPDC instead of the weak coherent pulse. The photon pair preparation process is shown in Figure 11(b). After a continuous laser with a center wavelength of $1555.8 \mathrm{~nm}$ is amplified by EDFA power, a continuous light of $777.9 \mathrm{~nm}$ is generated by a frequency doubling process in a PPLN waveguide. The SPDC photon pair is prepared by pumping the second waveguide with the frequency-doubled 
(a)

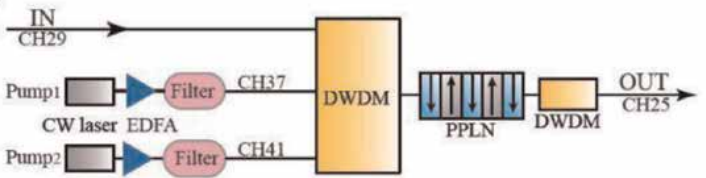

(b)

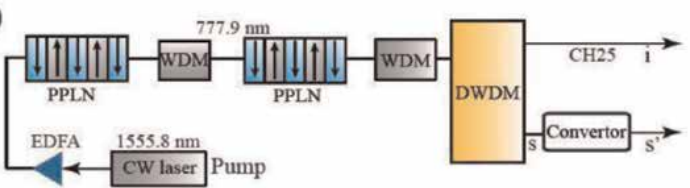

(c)

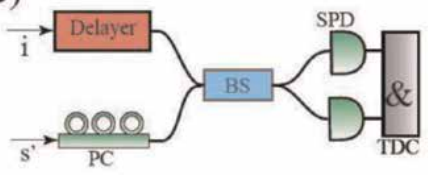

(d)

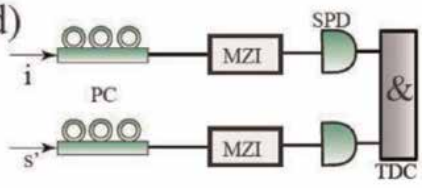

Figure 11.

Experimental setup of (a) the single-photon frequency convertor, $(b)$ photon-pairs preparation, (c) Hong-Ou-Mandel interference, and (d) measurement of time-energy entanglement. DWDM, 10o-GHz dense wavelength-division multiplexing; $\mathrm{CH}_{2} 5$ and $\mathrm{CH}_{3} 7$ and $\mathrm{CH}_{41}, \mathrm{DWDM}$ channels with 100-GHz spacing defined by ITU-TG.694.1; Filter, combination of DWDM and band pass filter (200-1540 and 1560$1800 \mathrm{~nm}$ ); PC, polarization controller; SPD, single-photon detector (quantum efficiencies, $\eta_{d}=10.0 \pm 0.2 \%$; repetition frequency of gate, $f=50 \mathrm{MHz}$; width of gate, $1 \mathrm{~ns}$; dark count probability per nanosecond, $\left.D=1 \times 10^{-6}\right)$; EDFA, erbium-doped fiber amplifier; WDM, 780-1550-nm wavelength-division multiplexing; TDC, time-to-digital convertor (coincidence time window, $t=1 \mathrm{~ns}$ ); Delayer, fiber path-length delayer; BS, 50:50 fiber beam splitter; MZI, 1-GHz unbalanced planar lightwave circuit Mach-Zehnder interferometers [7].

continuous light. WDM with an isolation of $180 \mathrm{~dB}$ is used to filter out noise. Finally, the corresponding two channels in signal (1554.13 nm, CH29) and idler (1557.36 nm, CH25) DWDM are separated.

First, we use classical light to test the conversion efficiency of tunable frequency conversion. By changing the center wavelength of the auxiliary pump P2, we achieved frequency conversion of the signal photon \pm 12 DWDM channels. In the classic light test, we set the signal power to $1 \mathrm{~mW}$, and both P1 and P2 have a power of $10 \mathrm{~mW}$ (because the PPLN waveguide is limited by thermal effects, the maximum total input power of the waveguide we use for conversion is around $20 \mathrm{~mW}$ ). We can get that the theoretical value is in good agreement with the experimental value, and the conversion efficiency of $\pm 12 \mathrm{CH}$ is about $0.8 \%$. In theory, the tunable frequency conversion has a full width at half maximum of about $76 \mathrm{~nm}$, which covers the entire communication C-band.

In our experiment, the photon-pair generation rate is set to 0.002 per detection gate. The maximum single-photon conversion efficiency in the experiment is that when $\mathrm{P}_{\mathrm{P} 1}=\mathrm{P}_{\mathrm{P} 2}=10 \mathrm{~mW}$, the maximum number of converted photons is $5.5 \times 10^{4} / \mathrm{s}$, and the conversion efficiency is calculated to be $0.55 \%$. At the same time, we measured that the noise generated by the frequency conversion process is $10^{-7}$ /gate. When the incident power of both auxiliary pumps is $179.5 \mathrm{~mW}, 100 \%$ conversion efficiency can be obtained. However, there are three main reasons for the reduction in conversion efficiency in actual experiments. The first reason is that the thermal effect of the PPLN waveguide limits the power of the incident light. This limitation is also the most important cause of the drop in conversion efficiency. We know that if a PPLN waveguide doped with $\mathrm{MgO}$ is used, the damage threshold can be greatly improved without changing the scattering properties of its refractive index, and it can withstand the total incident power of $360 \mathrm{~mW}$. Another reason is the phase difference between the two auxiliary pumps P1 and P2, and we get the conversion efficiency proportional to $\left|\varphi_{1}-\varphi_{2}\right|$. Since there is no synchronous lock between the phase differences between P1 and P2 in our experiments, $\left|\varphi_{1}-\varphi_{2}\right|$ is equal to 0.5 after averaging over time. This reason will directly lead to a $50 \%$ reduction in conversion efficiency. The last reason is also the problem that 
other frequency conversion methods will encounter, namely, the loss of fiber coupling and the loss caused by the filter. In our experiment, the total propagation and coupling loss in the PPLN waveguide and the filters is only $4.9 \mathrm{~dB}$.

After measuring the maximum conversion efficiency experimentally, we measured the conversion accuracy of our single-photon frequency converter by HOM interference. The experimental setup for HOM interference is shown in Figure 11(c) [14], where $i^{\prime}$ is the idler photon in the photon pair and $\mathrm{s}^{\prime}$ represents the converted signal photon. We placed a fiber optic delay in the i-photon beam path (Delayer, Delayer has an adjustment accuracy of $0.02 \mathrm{~mm}$.), Delayer is used to change the optical path difference $\delta x$ between $i$ and $s^{\prime}$; placing polarization control in the $s^{\prime}$ photon path (PC), PC is used to change the polarization of the $\mathrm{s}^{\prime}$ photon to match the polarization of the i-photon, because the HOM interference visibility of the identical particles is best. In our experiments, the ratio of BS transmittance to reflectance used in our experiments was measured as $\mathrm{T}: \mathrm{R}=49.9: 50.1$. The single-photon detector used in this experiment is still a pulse gate detection method, but the performance is upgraded. The dark counts of both detectors are $D=1 \times 10^{-6}$ per gate.

In HOM, we set the photon pair generation rate $\mu=0.002$ per gate; the single photon conversion efficiency is set to the maximum conversion efficiency under the constraint condition, i.e., $0.55 \%$; and the measurement time of each data point is $1000 \mathrm{~s}$. Under these conditions, the HOM interference curve we measured is shown in Figure 12. The calculated HOM interference visibility of the converted photon pair is $(80.5 \pm 3.5) \%$, and this contrast is much larger than the classical and nonclassical limit of $50 \%$, which proves that our single photon converter does. We further analyzed the HOM interference gram and found that the full width at half maximum of the interference gram is $0.56 \mathrm{~mm}$, and the corresponding time is $0.28 \mathrm{ps}$. Our theoretical full width at half maximum is about $0.50 \mathrm{~mm}$; thus, the theoretical and experimental results are more consistent.

In addition to the true conversion of this feature, another important feature of single-photon frequency conversion is the preservation of quantum properties. As with some previous quantum frequency converters, we demonstrate that the quantum properties are not corrupted during the conversion process by measuring the quantum state visibility of the photon pairs before and after the conversion. In our experiments, the signal and idler photon pairs were prepared as time-energy entangled photon pairs. The time-energy entanglement pair is generated by a

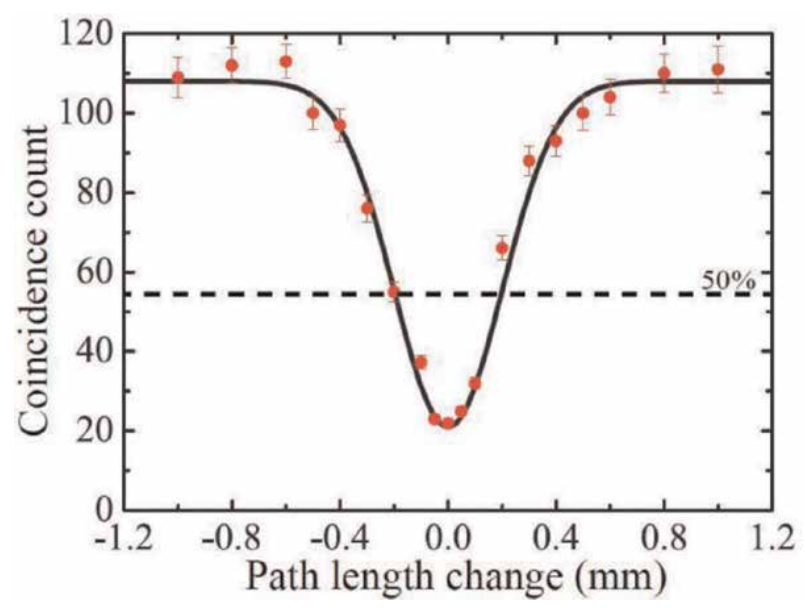

Figure 12.

Coincidence count as a function of the path-length change of one photon. The standard deviation is calculated by assuming a Poisson distribution of photon counts. The dashed horizontal line at $50 \%$ is the dividing line between the classical and nonclassical interference [7]. 
continuous laser (cw-laser) pumping the PPLN waveguide through the SPDC process [15]. Because the coherence time $\tau_{1}$ of cw-laser is extremely long, and the bandwidth of the down-converted photon pair is much larger than the bandwidth of the pump photon, that is, the coherence time $\tau_{2}$ between photon pairs is very short.

To measure the characteristics of time-energy entanglement and to exploit this entanglement, we use two unbalanced Mach-Zehnder interferometers (MZI) as shown in Figure 11(d), connecting a single photon detector at the output of the MZI, measuring two coincidence count at the output. We define the optical path difference of the two paths of the MZI interferometer as $\tau_{3}$. It is assumed that when $\tau_{1} \geq \tau_{3}>\tau_{2}$, the exit end of MZI does not have a single photon interference image, and the image of two-photon interference can be observed by the coincidence measurement.

The MZI used in our experiments is to change the phase difference between the long and short arms by adjusting the temperature. The phase change of $2 \pi$ corresponds to a temperature change of $0.7^{\circ}$. The entangled interference image before single-photon frequency conversion is shown in Figure 13. Because it is necessary to observe the interference image under the two non-collinear base vectors, we can prove that the two-photon is entangled, so we set the MZI temperature of the signal
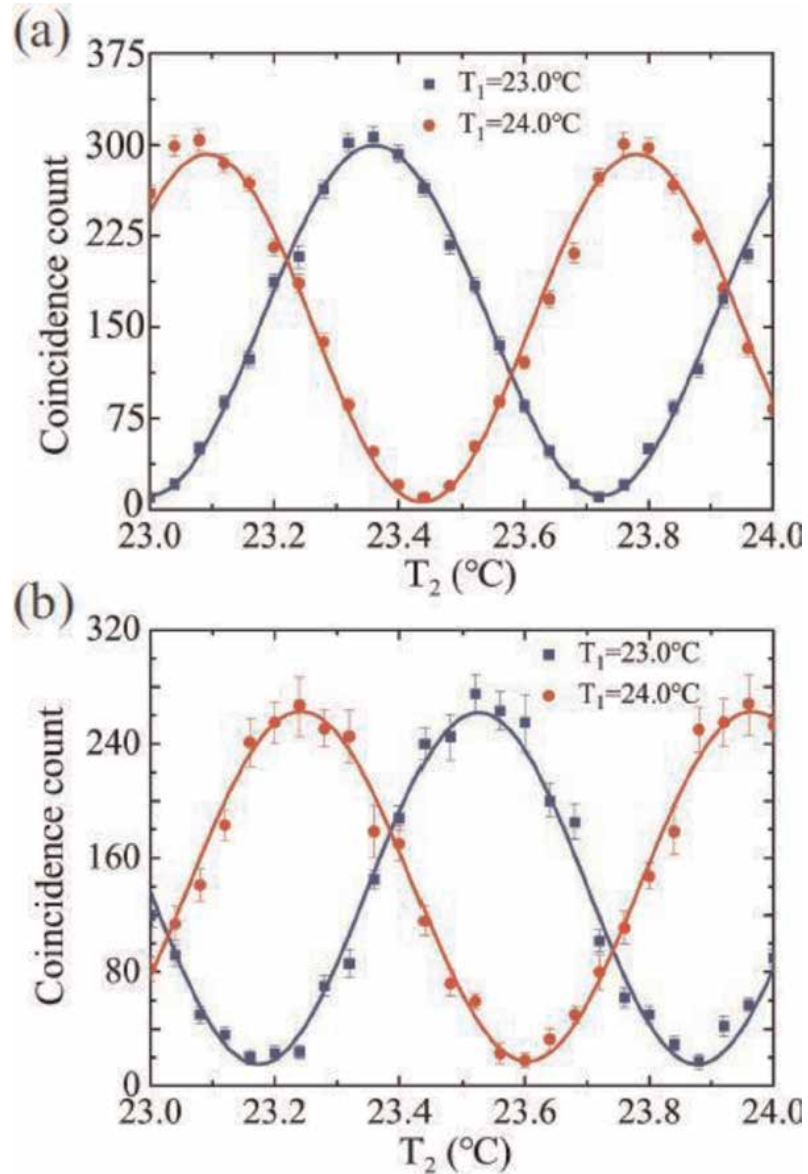

Figure 13.

Two-photon interference pattern (a) before and $(b)$ after the frequency conversion. $T_{1}$ is the temperature of the MZI in the signal channel, and $T_{2}$ is the temperature in the idler channel. The integration time for each dot is (a) $15 \mathrm{~s}$ and (b) $3000 \mathrm{~s}[\mathrm{7}]$. 
channel to 23.0 and $24.0^{\circ}$, respectively. The collinear base vector is measured. From Figure 13(a), we calculated the average pre-conversion entanglement visibility as $V=(93.8 \pm 1.6) \%$.

After the single photon frequency conversion, we again measure the entanglement visibility of the converted photon pair, as shown in Figure 13(b). It is calculated that the converted entanglement visibility is $V=(88.2 \pm 5.1) \%$, and this visibility can still break the $71 \%$ visibility of Bell's inequality. Therefore, the $88.2 \%$ visibility after conversion proves that our single-photon frequency converter maintains the quantum properties of photons with a theoretical visibility of $91 \%$. Since the converted count efficiency is low, the measurement time is extended from $15 \mathrm{~s}$ before conversion to $1000 \mathrm{~s}$. The result convincingly shows that quantum entanglement is well preserved during the frequency conversion. Thus, the photon pairs can still be used for quantum communication tasks. We expect that our scheme may have applications in quantum systems, such as quantum communication on multiuser fiber quantum networks and quantum cryptography with independent single photon sources.

\section{Conclusion}

We have demonstrated three kinds of quantum interfaces with different functions. First, we have experimentally demonstrated that the spectrum of singlephoton-level laser pulse was compressed by a factor of 58 in a PPLN waveguide chip, where a chirped single-photon-level laser pulse and an antichirped laser pulse by fiber Bragg gratings are used to achieve a pulse with new frequency through SFG. Our results have demonstrated the potentially application for PPLN waveguide chip as an integrated platform for spectrum compressing and frequency conversing in the telecom band, such as coherent photonic interfaces between quantum communication at $1550 \mathrm{~nm}$ and quantum memory in the near-visible window. Second, we have realized high efficiency SFG between two broadband single-photon-level coherent states by using a high-efficiency PPLN waveguide chip. The result is already competitive with methods based on linear optics, and offers new possibilities such as heralding entanglement at a distance. This technique in our proposal marks a critical step toward the implementation of DI-QKD. Final, we have demonstrated single-photon frequency conversion using a cascaded quadratic nonlinearity in PPLN waveguides chip. The clear HOM dip observed in our experiment shows that the frequency has been precisely switched between DWDM channels. Moreover, the time-energy entanglement is well preserved during the frequency conversion. All works above are of great significance to the development of quantum optics.

\section{Conflict of interest}

The authors declare no conflict of interest. 


\section{Author details}

Yuanhua $\mathrm{Li}^{1 *}$ and Xianfeng $\mathrm{Chen}^{2}$

1 Department of Physics, Jiangxi Normal University, Nanchang, China

2 State Key Laboratory of Advanced Optical Communication Systems and Networks, Department of Physics and Astronomy, Shanghai Jiao Tong University, Shanghai, China

*Address all correspondence to: lyhua1984@163.com

\section{IntechOpen}

(C) 2019 The Author(s). Licensee IntechOpen. This chapter is distributed under the terms of the Creative Commons Attribution License (http://creativecommons.org/licenses/ by/3.0), which permits unrestricted use, distribution, and reproduction in any medium, provided the original work is properly cited. (cc)BY 


\section{References}

[1] Sun QC, Mao YL, Chen SJ, Zhang W, Jiang YF, Zhang YB, et al. Quantum teleportation with independent sources and prior entanglement distribution over a network. Nature Photonics. 2016; 10(10):671-675. DOI: 10.1038/ nphoton.2016.179

[2] Miller J, Miyake A. Resource quality of a symmetry-protected topologically ordered phase for quantum computation. Physical Review Letters. 2015;114(12):120506. DOI: 10.1103/ PhysRevLett.114.120506

[3] Specht HP, Nölleke C, Reiserer A, Uphoff M, Figueroa E, Ritter S, et al. A single-atom quantum memory. Nature. 2011;473(7346):190-193. DOI: 10.1038/ nature09997

[4] Yurke B, Denker JS. Quantum network theory. Physical Review A. 1984;29(3):1419. DOI: 10.1103/ PhysRevA.29.1419

[5] Li YH, Xiang T, Nie YY, Sang MH, Chen XF. Spectral compression of single-photon-level laser pulse. Scientific Reports. 2017;7:43494. DOI: 10.1038/srep43494

[6] Li YH, Xiang T, Nie YY, Sang MH, Chen XF. Nonlinear interaction between broadband single-photon-level coherent states. Photonics Research. 2017;5(4): 324-328. DOI: 10.1364/PRJ.5.000324

[7] Xiang T, Sun QC, Li YH, Zheng YL, Chen XF. Single-photon frequency conversion via cascaded quadratic nonlinear processes. Physical Review A. 2018;97(6):063810. DOI: 10.1103/ PhysRevA.97.063810

[8] Lavoie J, Donohue JM, Wright LG, Fedrizzi A, Resch KJ. Spectral compression of single photons. Nature Photonics. 2013;7(5):363-366. DOI: 10.1038/nphoton.2013.47
[9] Thew RT, Zbinden H, Gisin N. Tunable upconversion photon detector. Applied Physics Letters. 2008;93(7): 071104. DOI: 10.1063/1.2969067

[10] Shalm LK, Hamel DR, Yan Z, Simon C, Resch KJ, Jennewein T. Threephoton energy-time entanglement. Nature Physics. 2013;9(1):19-22. DOI: $10.1038 /$ nphys 2492

[11] Matsuda N, Shimizu R, Mitsumori Y, Kosaka H, Edamatsu K. Observation of optical-fibre Kerr nonlinearity at the single-photon level. Nature Photonics. 2009;3(2):95-98.

DOI: $10.1038 /$ nphoton.2008.292

[12] Sangouard N, Sanguinetti B, Curtz N, Gisin N, Thew R, Zbinden H. Faithful entanglement swapping based on sum-frequency generation. Physical Review Letters. 2011;106(12):120403. DOI: 10.1103/PhysRevLett.106.120403

[13] Kumar P. Quantum frequency conversion. Optics Letters. 1990;15(24): 1476-1478. DOI: 10.1364/OL.15.001476

[14] Hong CK, Ou ZY, Mandel L. Measurement of subpicosecond time intervals between two photons by interference. Physical Review Letters. 1987;59(18):2044-2046. DOI: 10.1103/ PhysRevLett.59.2044

[15] Franson JD. Bell inequality for position and time. Physical Review Letters. 1989;62(19):2205-2208. DOI: 10.1103/PhysRevLett.62.2205 
Section 3

Nonreciprocal Control 



\title{
Optical Chirality and Single-Photon Isolation
}

\author{
Lei Tang and Keyu Xia
}

\begin{abstract}
Optical isolation is important for protecting a laser from damage due to the detrimental back reflection of light. It typically relies on breaking Lorentz reciprocity and normally is achieved via the Faraday magneto-optical effect, requiring a strong external magnetic field. Single-photon isolation, the quantum counterpart of optical isolation, is the key functional component in quantum information processing, but its realization is challenging. In this chapter, we present all-optical schemes for isolating the backscattering from single photons. In the first scheme, we show the single-photon isolation can be realized by using a chiral quantum optical system, in which a quantum emitter asymmetrically couples to nanowaveguide modes or whispering-gallery modes with high optical chirality. Secondly, we propose a chiral optical Kerr nonlinearity to bypass the so-called dynamical reciprocity in nonlinear optics and then achieve room-temperature photon isolation with low insertion loss. The concepts we present may pave the way for quantum information processing in an unconventional way.
\end{abstract}

Keywords: single-photon isolation, optical chirality, chiral light-matter interaction, optical isolator, optical circulator, chiral Kerr nonlinearity

\section{Introduction}

Controlling the flow of light is extremely essential for quantum information processing in integrated optical circuits. Nonreciprocal propagation of light at the single-photon level is in great demand for applications in quantum networks $[1,2]$, quantum computing [3], quantum entanglement [4], and quantum measurement [5]. For this purpose, nonreciprocal photonic elements, such as optical isolators and circulators, processing and routing of photonic signals at ultralow light level, or single-photon level in integrated optical circuits has been attracting a lot of interest.

The conventional implementations of nonreciprocal optical devices are achieved by using the Faraday magneto-optical effect. However, such Faraday-effect-based devices suffer large optical losses and conflict with miniaturization and integration. To date, integrated nonreciprocal photonic elements have been demonstrated via magneto-optical effect [6, 7], optical nonlinearity [8-10], and opto-mechanical system [11-13]. Very recently, Dong et al. proposed and experimentally realized a scheme to achieve a true single-photon non-reciprocity in a cold atomic ensemble [14]. However, most of these devices cannot achieve high isolations, low losses, and compatibility with single-photon level at the same time. 
Nanophotonic devices control and confine the flow of light at a subwavelength scale. The strong confinement in these structures yields optical chirality, which is an inherent link between local polarization and the propagating direction of light [15]. If quantum emitters are embedded in these structures, chiral light-matter interaction is obtained, leading to propagation-direction-dependent emission, absorption, and scattering of photons. As a result, chiral light-matter interaction can be used to break time symmetry and achieve on-chip single-photon isolation. Some feasible schemes based on chiral quantum optics have been proposed to realize nonreciprocity at the single-photon level $[16,17]$, and optical isolators and circulators have been experimentally demonstrated in full quantum regime $[18,19]$.

Besides strong confinement of light, atoms can induce optical chirality. Here, optical chirality is chiral cross-Kerr (XKerr) nonlinearity induced in atoms. As a result of the chirality of atomic nonlinearity, the phases and transmission amplitudes of the forward- and backward-moving probe fields are sufficiently different after passing through atoms in two opposite directions. Thus, chiral XKerr nonlinear can achieve chip-compatible optical isolation with high isolation and low insert losses [20]. And very recently, XKerr-based optical isolators and circulators for high isolation, low loss, and an ultralow probe field at room temperature have been experimentally demonstrated [21].

\section{Optical chirality and chiral light-matter interaction}

The strong light confinement in subwavelength structures, e.g., nanofibers, nanowaveguides, or whispering-gallery mode (WGM) microresonators, can lock the local polarization of the light to its propagation direction. In these structures, the light is strongly confined transversely, leading to a longitudinal component of the electric field (e-field), which is parallel with the propagation direction. The longitudinal and transverse components, denoted as $\mathbf{E}_{\|}$and $\mathbf{E}_{\perp}$, respectively, are comparable, and the former oscillates $\pm \pi / 2$ radians out of phase with respect to the latter, with the \pm sign depending on the propagation direction of the light (forward or backward) [15]:

$$
\boldsymbol{E}_{\text {local }}=\boldsymbol{E}_{\perp} \pm i \boldsymbol{E}_{\|} .
$$

As a consequence, the local polarization of the light is elliptical, yielding a transverse spin angular momentum component, whose e-field rotates around an axis perpendicular to the propagation direction. The transverse spin components flip sign when the propagation of light reverses. This correlation of the polarization and the propagation direction is named the spin-momentum locking (SML) [15]. For the ideal case, $\left|\boldsymbol{E}_{\perp}\right|=\left|\boldsymbol{E}_{\|}\right|$, the e-field is circularly polarized.

In order to characterize what degree the e-field is locked to the momentum or what percentage of circular polarization of the local light in the nanostructures, the circular polarization unit vectors are defined as

$$
\hat{\boldsymbol{\sigma}}^{ \pm}=\frac{\hat{\mathbf{x}} \pm i \hat{\mathbf{y}}}{\sqrt{2}}
$$

where $\hat{\boldsymbol{\sigma}}$ is for the right circularly polarized unit vector and $\hat{\boldsymbol{\sigma}}^{-}$for the left circularly polarized one and $\hat{\mathbf{x}}$ and $\hat{\mathbf{y}}$ are unit vectors along the $\mathrm{x}$ and $\mathrm{y}$ directions. Thus, the optical chirality (OC) of an e-field is defined as $[17,22]$ :

$$
\mathcal{C}=\frac{\left|E(r) \cdot \hat{\sigma}^{-}\right|^{2}-\left|E(r) \cdot \hat{\sigma}^{+}\right|^{2}}{|E(r)|^{2}} .
$$


Obviously, the OC is limited to a region from -1 to 1 . Note that the value $\mathcal{C}=$ $1(-1)$ implies the e-field is entirely $\sigma^{-}-\left(\sigma^{+}-\right)$polarized, while $\mathcal{C}=0$ corresponds to a linear polarization. The intensity difference between the right circularly $\left(\sigma^{+}\right)$and the left circularly $\left(\sigma^{-}\right)$polarized components at the position $r$ is calculated by $\mathcal{D}=$ $\left|\mathbf{E}(\mathbf{r}) \cdot \hat{\sigma}^{-}\right|^{2}-\left|\mathbf{E}(\mathbf{r}) \cdot \hat{\sigma}^{+}\right|^{2}$.

Then we focus on the interaction between the light possessing photonic SML and chiral quantum emitters with polarization-dependent dipole transitions. If a pair of counter-propagating spin-momentum-locked light interacts with quantum emitters, the interaction becomes chiral. In other words, the interaction strength for forward-and backward-propagating light modes is different. In this case, photon emission, absorption, and scattering become unidirectional. As a result, optical nonreciprocal flow of light can be achieved when a quantum emitter with degenerate transitions is populated in a specific spin state or one can shift the transition energy to make it couple (decouple) with one of the two of counter-propagating modes. On the basis of these effects, optical isolation can be realized at the singlephoton level, which enables nonreciprocal single-photon devices, e.g., singlephoton isolator and circulator. Next, we introduce the realization of single-photon isolators and circulators based on chiral light-matter interaction.

\section{Single-photon isolation using chiral light-matter interaction}

A single-photon isolator and circulator can be achieved by chirally coupling a quantum emitter to a passive, linear nanophotonic waveguide or a WGM microresonator which possesses optical chirality.

\subsection{Single-photon isolator based on a nanophotonic waveguide}

The type-I single-photon isolator is based on a line defect photonic crystal waveguide [16]. By carefully engineering the photonic crystal waveguide, it can have an in-plane circular polarization, and counter-propagating modes are counter circulating [22]. A quantum emitter is doped at the position where the waveguide possesses only the right-propagating $\sigma^{+}$-polarized light or left-propagating $\sigma^{-}$polarized light, as shown in Figure 1a. The doped emitter strongly interacts with the $\sigma^{+}$-polarized light but weakly couples to the $\sigma^{-}$-polarized light. As a result, the time-reverse symmetry of the waveguide-emitter system breaks. The emitter scatters the forward- (right-) propagating single photons into an open environment whereas behaves transparently for the backward- (left-) propagating photons.

The steady-state transmission for the two atomic transitions coupling with waveguide is calculated by using the photon transport method [16, 23, 24]

$$
t_{ \pm}(\omega)=\frac{\omega-\omega_{q}-i\left(\gamma_{ \pm}-\Gamma_{ \pm}\right)}{\omega-\omega_{q}+i\left(\gamma_{ \pm}-\Gamma_{ \pm}\right)}
$$

where $\omega$ is the frequency of the input photon and $\omega_{q}$ is the frequency of the atomic transition. The detuning is defined as $\Delta=\omega_{q}-\omega$. In $\Gamma_{ \pm}=V_{ \pm}^{2} / 2 v_{g}, V_{ \pm}$is the coupling strength between the atom and the field in the waveguide, which is proportional to the atomic dipole moments $\mu_{ \pm}$, and $v_{g}$ is the group velocity of the photon in the waveguide. A Cs atom is used as the quantum emitter so that $\mu_{+}=\sqrt{45} \mu_{-}$, and $\gamma_{ \pm}$, $\Gamma_{ \pm} \propto\left|\mu_{ \pm}\right|^{2}$. As a consequence, $\gamma_{+} / \gamma_{-}=\Gamma_{+} / \Gamma_{-} \gg 1$. The transmission is defined as $T_{ \pm}=\left|t_{ \pm}\right|^{2}$. The isolation contrast is evaluated as $\mathbf{Y}=\left(T_{+}-T_{-}\right) /\left(T_{+}+T_{-}\right)$[23]. 


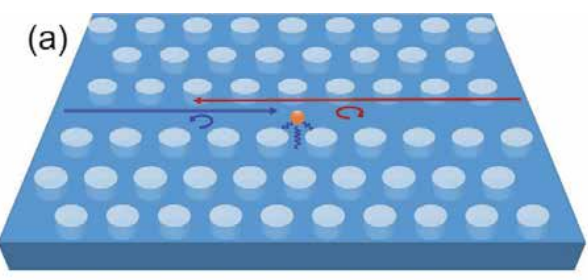

(b)

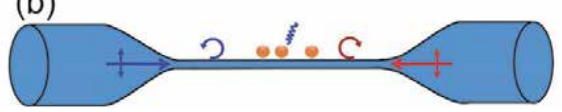

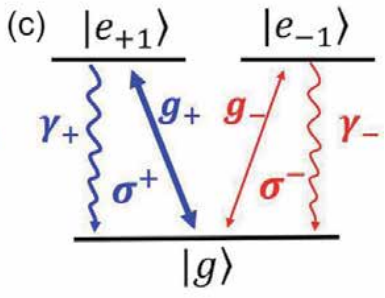

Figure 1.

Schematics of the single-photon isolators. (a) Single-photon isolator based on a photonic crystal waveguide asymmetrically coupling with a quantum emitter. The waveguide possesses local circular polarization, and its rotating direction is dependent on the propagating direction [16]. The quantum emitter is doped in these specific sites. (b) Single-photon isolator based on a photonic nanofiber asymmetrically coupling with quantum emitters. The nanofiber is realized as the waist of a tapered silica fiber, whose evanescent fields exhibit propagationdirection-dependent circular polarization [18]. Quantum emitters are located in the vicinity of the nanofiber. (c) Energy-level diagram for a quantum emitter with unbalanced decay rates $\gamma_{+} \gg \gamma_{-}$and different coupling strengths with $\sigma^{ \pm}$-polarized light $g_{+} \gg g_{-}$. The states $\left|e_{ \pm 1}\right\rangle$ and $|g\rangle$ correspond to excited states and a ground state, respectively, and the transition $|g\rangle \leftrightarrow\left|e_{+1}\right\rangle$ is driven by $\sigma^{+}$-polarized light, while $|g\rangle \leftrightarrow\left|e_{-1}\right\rangle$ transition is driven by $\sigma^{-}$-polarized light. Here, quantum emitters can be Cs atoms [25], Rb atoms [26], or quantum dots [27].

Due to the coupling of the atom to the waveguide and open environment, the ratio of atomic dissipation rate is set to $\alpha=\Gamma_{+} / \gamma_{+}=\Gamma_{-} / \gamma_{-}$. If $\alpha \approx 1$, the singlephoton isolation is achieved, as shown in Figure 2a. Obviously, $T_{-}$is almost equal to unity for a right-hand input, while $T_{+}$is small if $10 \leqslant|\Delta| / \gamma_{-} \leqslant 30$. Note that the transmissions are the same, $\sim 0$ at $\Delta=0$ for both of the right-handed and lefthanded inputs. At vanishing detuning, irrespective of the propagating direction, photons cannot be transmitted through the waveguide. However, the linewidths of the dips for the left-handed input (blue solid line) are much broader than those for the right-handed input (red dashed line). Because of this directionality-dependent linewidth, a single-photon wave packet with a duration $\Gamma_{+}^{-1} \ll \tau \ll \Gamma_{-}^{-1}$ when incident from the right is mostly transmitted through the waveguide, while when it is incident from the left, it is mostly scattered away to the open environment. Note that the performance of the single-photon isolation is dependent on the ratio $\alpha$. As seen from Figure $\mathbf{2 b}$, a good nonreciprocal behavior occurs only around the critical coupling $\alpha \approx 1$, and if $5 \alpha \leqslant|\Delta| / \gamma_{-} \leqslant 10 \alpha$ and $0.53<\alpha<1.8$, the isolation contrast $\Upsilon$ can be larger than 0.8 .

Low-loss silica nanophotonic waveguides with a strongly nonreciprocal transmission controlled by the internal state of spin-polarized atoms have been
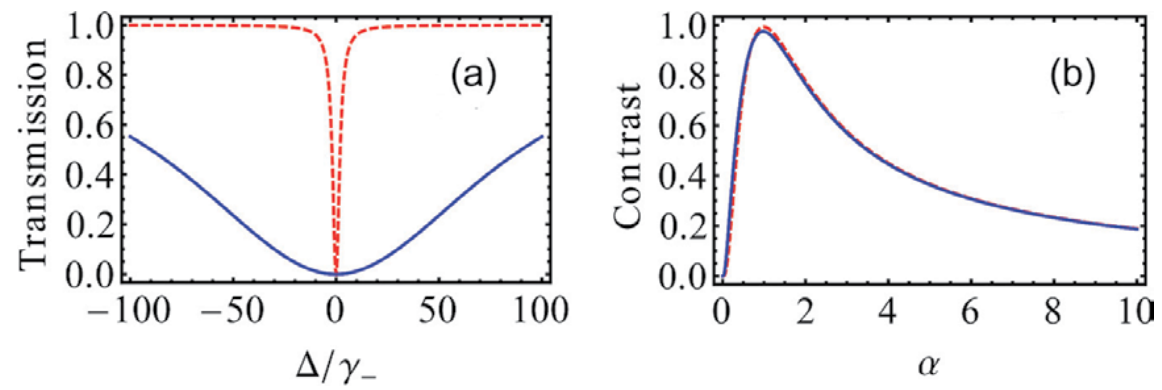

Figure 2.

(a) Steady-state transmission as a function of $\Delta$ for $\alpha=1$. Solid blue curve is for transmission $T_{+}$, and dashed red curve is for transmission $T_{-}$. (b) Isolation contrast as a function of $\alpha$. Solid blue curve indicates $r$ for $|\Delta| / \gamma_{-}=10 \alpha$ and dashed red curve for $|\Delta| / \gamma_{-}=5 \alpha$. The two curves are mostly overlapped. Figures are reproduced with permission from [16]. 
demonstrated [18]. In the experiment, an ensemble of individual cesium atoms is located in the vicinity of a subwavelength-diameter silica nanofiber (250-nm radius) trapped in a nanofiber-based two-color optical dipole trap [28]. As shown in Figure 1b, a quasilinearly polarized light incident to the nanofiber exhibits chiral character at the position of the atoms: when the evanescent field propagates in the forward direction, it is almost fully $\sigma^{+}$-polarized, while it is almost fully $\sigma^{-}$-polarized if it propagates in the backward direction [18]. The experimental results show that the probe light with a power of $0.8 \mathrm{pW}$, corresponding to about 0.1 photon per excited-state lifetime, incident into nanofiber from different port obtains nonreciprocal transmissions, $T_{+}=0.13 \pm 0.01$ and $T_{-}=$ $0.78 \pm 0.02$, yielding an isolation $\mathcal{F}=10\left|\log \left(T_{-} / T_{+}\right)\right|=7.8 \mathrm{~dB}$ [18]. Furthermore, trapping more atoms in the vicinity of the nanofiber can increase the isolation [18].

\subsection{Single-photon isolator and circulator based on a WGM microresonator}

The type-II single-photon isolator is based on a WGM microresonator. In this setup, when the linear polarized light enters the bus-waveguide from port $P_{1}$, it excites a $\sigma^{+}$-polarized counterclockwise (CCW) mode, while when it is incident from port $P_{2}$, it drives $\sigma^{-}$-polarized clockwise (CW) mode [16]. As a result, the two counter-propagating modes in the WGM microresonator couple to a quantum emitter with different dipole moments $\mu_{+}$and $\mu_{-}$corresponding to coupling strength $g_{+}$and $g_{-}$.

The transmission into the bus and drop waveguides are calculated by [16].

$$
\begin{gathered}
t_{ \pm, B}(\omega)=1+\frac{2 i \kappa_{e x 1}}{\omega_{c}-\omega-i \kappa-\frac{\left|g_{ \pm}\right|^{2}}{\omega_{q}-\omega-i \gamma_{ \pm}}}, \\
t_{ \pm, D}(\omega)=\frac{2 i \sqrt{\kappa_{e x 1} \kappa_{e x 2}}}{\omega_{c}-\omega-i \kappa-\frac{\left|g_{ \pm}\right|^{2}}{\omega_{q}-\omega-i \gamma_{ \pm}}}
\end{gathered}
$$

where $\kappa_{\text {ex1(2) }}=V_{1(2)}^{2} / 2 v_{g}$ is the decay rate of the resonator due to the external coupling $V_{1(2)}$ to the bus (drop) waveguide, and the photons in both of the bus and drop waveguides have the same group velocity $v_{g}$ assumed. The total decay rate of the resonator is $\kappa=\kappa_{i}+\kappa_{\text {ex } 1}+\kappa_{\text {ex2 } 2}$ where $\kappa_{i}$ is the intrinsic decay rate of the resonator. The detuning is defined as $\Delta=\omega_{c}-\omega$, and $\omega_{c}=\omega_{q}$ is assumed where $\omega_{c}$ is the resonating frequency of the resonator and $\omega_{q}$ is the transition frequency of the quantum emitter.

In the configuration of this device, if the drop waveguide is removed, i.e., $\kappa_{e \times 2}=0$, single-photon isolator is achieved (see Figure 3 ). In this case, $T_{ \pm, B}$ is substituted with $T_{ \pm}$. As shown in Figure 4, if $g_{-}=0$ and $g_{+} \gg \kappa_{i}$, at $\Delta=0, T_{+}=1$, while $T_{-} \approx 0$. This can be achieved by using a negatively charged quantum dot as the quantum emitter [27]. If $\left|g_{-}\right|>0$ and $\Delta \approx 0$, the nonreciprocal window disappears. However, when $|\Delta| \approx\left|g_{-}\right|, \gamma_{+}=0$, and $g_{+} / \kappa_{i} \gg 1$, the right-moving photon can pass through the device, corresponding to $T_{+}=1$ (solid blue curve), while the left-moving photon decays into the environment via the resonator, corresponding to $T_{-}=0$ (solid red curve). At $|\Delta| \approx\left|g_{+}\right|$, the optical nonreciprocity is reversed. The device is transparent for left-moving photon but blocks the right-moving photon. If $\gamma_{+} \gg \kappa_{i}$, the part of the excitation of the right-moving photon can pass through the device (dotted green curve). 


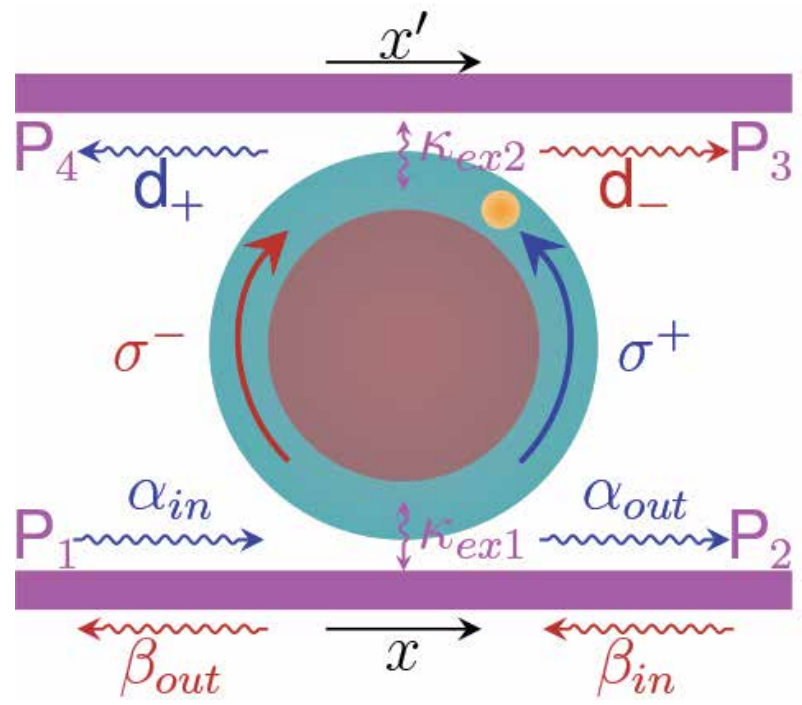

Figure 3.

Schematic of the single-photon isolator or circulator. There is a quantum emitter doped in a WGM microresonator, which possesses a $\sigma^{+}$-polarized CCW mode and a $\sigma^{-}$-polarized CW mode. The microresonator couples to a lower bus waveguide with a rate $\kappa_{\text {ex1 }}$ and a upper drop waveguide with a rate $\kappa_{\text {ex } 2}$. Reproduced with permission from [16].

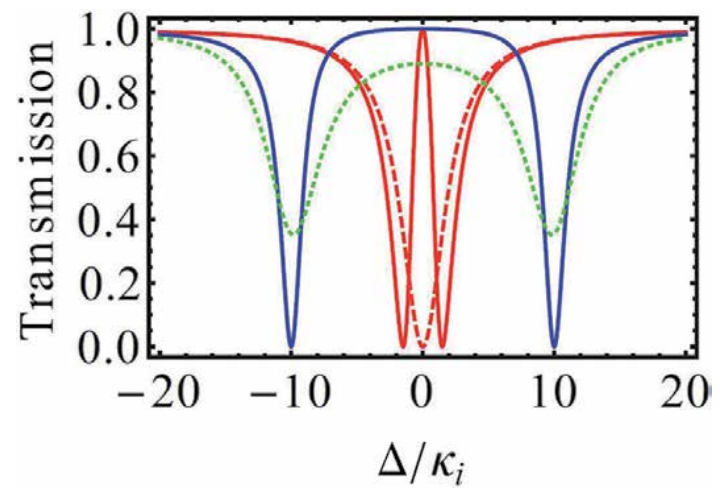

Figure 4.

Steady-state transmissions of the single-photon isolator using a WGM microresonator in the absence of the drop waveguide, i.e., $\kappa_{\text {exz }}=0$, and under the critical coupling condition $\kappa_{e x 1}=\kappa_{i}$. Dashed and solid curves are for the transmission $T_{-}$when $g_{-}=0$ and $g_{-}=g_{+} / \sqrt{45}$, while solid blue and dotted green curves are for the transmission $T_{+}$when $\gamma_{+}=0$ and $\gamma_{+}=3 \kappa_{i}$, respectively. $g_{+}=10 \kappa_{i}$. Reproduced with permission from [16].

A WGM bottle microresonator coupling to the optical fiber and a single ${ }^{85} \mathrm{Rb}$ atom to realize the type-II optical isolator has been demonstrated [18]. In the experiment, for the bottle microresonator, it sustains WGMs with ultrahigh quality factor and small mode volume [26]. As a result of strong transverse confined, its evanescent fields of WGMs are almost fully circularly polarized, with OC $\mathcal{C}>0.96$ [26]. The single ${ }^{85} \mathrm{Rb}$ atom is prepared in the outermost $m_{F}=3$ Zeeman substrate of the $F=3$ hyperfine ground state. As seen from Figure 1c, the states $\left|e_{+1}\right\rangle$ and $\left|e_{-1}\right\rangle$ correspond to the excited states $\left|F^{\prime}=4, m_{F^{\prime}}=+4\right\rangle$ and $\left|F^{\prime}=4, m_{F^{\prime}}=+2\right\rangle$, respectively. In the experiment, the transition $|g\rangle \rightarrow\left|e_{+1}\right\rangle$ is much stronger than the $|g\rangle \rightarrow$ $\left|e_{-1}\right\rangle$ transition, corresponding to the coupling strength, $g_{+}$and $g_{-}$, between the atom and the two counterrotating WGMs, yielding $g_{+} / g_{-}=5.8$ [18]. The experimental results show that when the probe light with power of $3 \mathrm{pW}$, corresponding 
to about 0.2 photon per resonator lifetime, obtains nonreciprocal transmissions through the system of $T_{+}=0.72 \pm 0.02$ in the forward direction and $T_{-}=$ $0.03 \pm 0.01$ in the backward direction, yielding an isolation $\mathcal{F}=13 \mathrm{~dB}$.

The single-photon circulator consists of two waveguides and a WGM microresonator, and both of the bus and drop waveguides overcouple to the resonator, i.e., $\kappa_{e x 1}=\kappa_{e x 2}=3 \kappa_{i}$ (see Figure 3). As seen from Figure 5a, at $\Delta \sim 0$, for port $P_{1}$ input $\alpha_{i n}$, most excitation of the photon can transport to the bus-waveguide port $P_{2}, T_{+, B}=0.85$ (solid blue curve), while the output to the drop-waveguide port $P_{4}$ is vanishingly small (dashed blue curve). As for port $P_{2}$ input $\beta_{i n}$, there is a dip (peak) in the transmission $T_{-, D}\left(T_{-, B}\right)$, but the linewidth is very small, $\sim 0.16 \kappa_{i}$, as shown in Figure 5a. In contrast, the whole transmission spectrum has a linewidth of $11 \kappa_{i}$. As a result, a single-photon pulse bandwidth $0.16 \kappa_{i} \ll \Delta B \ll 11 \kappa_{i}$ can transport to the drop-waveguide port $P_{3}$ with a probability of 0.74 . The probability of transmitting to the bus-waveguide port $P_{1}$ is small, $\sim 0.02$, yielding a contrast 0.95 . The numerical simulations of the propagation of a single-photon pulse are performed in [16], which match the analytic forms well (see gray curves in Figure 5a). If $|\Delta| \sim g_{-}$, $T_{+, B}=0.835$ to the $P_{2}, T_{+, D}=0.032$ to $P_{4}$, and $T_{-, B}=0.021$ to the $P_{1}, T_{-, D}=0.733$ to $P_{3}$. As a consequence, the device forms a $P_{1} \rightarrow P_{2} \rightarrow P_{3}$ circulator. The singlephoton incident from port $P_{1}$ transmits to port $P_{2}$, but the photon entering port $P_{2}$ comes out from port $P_{3}$.

If $\Delta \sim 0$, the numerical simulations of the propagation of a single-photon pulse in time as it passed through the circulator are performed in [16]. Figure $5 \mathbf{b}$ shows Gaussian pulse wave packets, $\phi(x, 0)_{ \pm, B}=\sqrt[4]{\tau^{2} / \pi} e^{-\left(x-x_{0}\right)^{2} / 2 \tau^{2}}$, incident from port $P_{1}$ and $P_{2}$ at the same time $t_{d}$. The transmissions are calculated by $T_{ \pm, B / D}=$ $\int_{-\infty}^{+\infty} \phi_{ \pm, B / D}^{*}\left(x, t_{d}\right) \phi\left(x, t_{d}\right) d x$ to be $\left\{T_{+, B}, T_{-, B}, T_{+, D}, T_{-, D}\right\}=\{0.9,0.178,0.02,0.675\}$ excitations output to port $\left\{P_{2}, P_{1}, P_{4}, P_{3}\right\}$. Obviously, a three-port circulator at the single-photon level is achieved.

Note that if the states are initially populated to $\left|6^{2} S_{1 / 2}, F=4, m=-4\right\rangle$ for Cs atoms or $\left|5^{2} S_{1 / 2}, F=3, m=-3\right\rangle$ for $\mathrm{Rb}$ atoms, $\mu_{+} \ll \mu_{-}$, the optical nonreciprocity can be reversed, and the single-photon circulator forms a $P_{2} \rightarrow P_{1} \rightarrow P_{4}$ circulator.
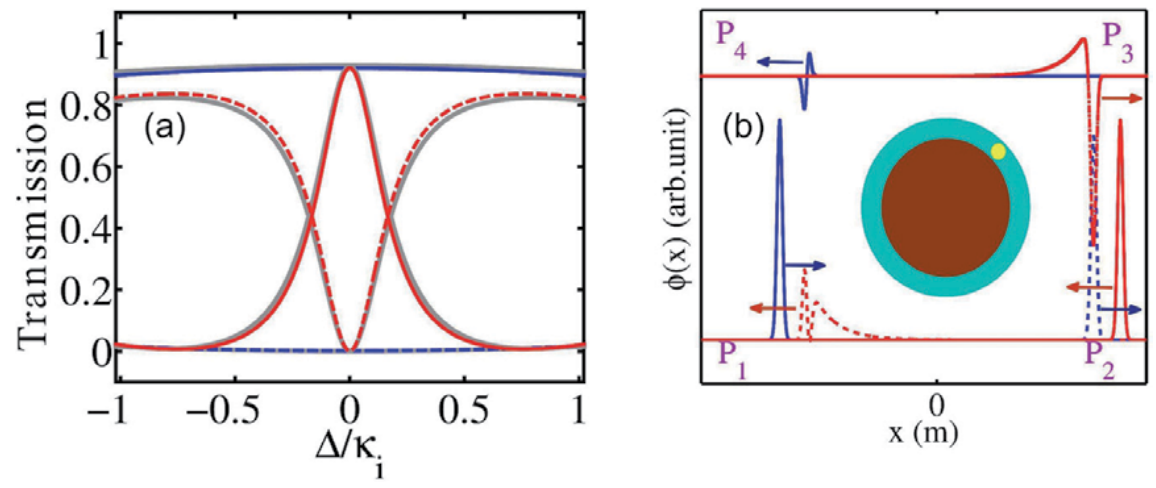

Figure 5.

(a) Steady-state transmissions of the single-photon circulator using a WGM microresonator and both of the bus and drop waveguides overcouple to the resonator, i.e., $\kappa_{\text {exx }}=\kappa_{\text {ex } 2}=3 \kappa_{\mathrm{i}}$. Solid blue (red) curve is for the transmission $T_{+, B}\left(T_{-, B}\right)$ in the bus waveguide, while the dashed blue (red) curve is for the transmission $T_{+, D}$ $\left(T_{-, D}\right)$ in the drop waveguide. The gray curves are the results from numerical simulations, which mostly overlap with other curves. $g_{+}=5 \kappa_{i}, g_{-}=g_{+} / \sqrt{45}, \gamma_{+}=0.3 \kappa_{i}$, and $\gamma_{-}=\gamma_{+} / 45$. (b) Propagation of singlephoton pulses with $\sim 4 \kappa_{i}$ in the resonator for $\Delta=0$ and $v_{g}=1 \times 10^{8} \mathrm{~m} / \mathrm{s}$. Blue (red) lines are for the input and transmitted excitations for the left-handed (right-handed) input. The arrows indicate the moving directions of photons. Reproduced with permission from [16]. 
A quantum optical circulator operated by a single atom has been demonstrated [19]. In the experiment, a single ${ }^{85} \mathrm{Rb}$ atom is coupled to the WGM of a bottle microresonator, which is interfaced by two tapered fiber couplers, realizing a fourport device. The single ${ }^{85} \mathrm{Rb}$ atom is prepared in the outermost Zeeman sublevel $m_{F}=+3$ of the $5 S_{1 / 2}, F=3$ hyperfine ground state. The light in the CCW mode excites state $\left|F^{\prime}=4, m_{F^{\prime}}=+4\right\rangle$ more strongly than CW mode exciting $\mid F^{\prime}=$ $\left.4, m_{F^{\prime}}=+2\right\rangle$ state, corresponding to coupling strength of resonator modes and the atom, $g_{+} \gg g_{-}$. The presence of the atom changes the resonator field decay rate from $\kappa_{\text {tot }}=\kappa_{i}+\kappa_{\text {ex } 1}+\kappa_{\text {ex2 } 2}$ to $\kappa_{\text {tot }}+\Gamma_{ \pm}$, where $\Gamma_{ \pm}=g_{ \pm}^{2} / \gamma$ is the direction-dependent atom-induced loss rate [19] and $\gamma=2 \pi \times 3 \mathrm{MHz}$ is the dipole decay rate of $\mathrm{Rb}$. For the $\mathrm{CW}$ mode, $\Gamma_{-}$is small, and the resonator field decay rate is not substantially modified by the atom, whereas for the CCW mode, $\Gamma_{+}$can become comparable with or even exceed $\kappa_{\text {tot }}$. As a result, when light is incident into the device from ports $P_{2}$ and $P_{4}$ for which it couples to the CW mode (see Figure 3), the add-drop functionality is achieved. However, for the two other input ports $P_{1}$ and $P_{3}$, the light couples to the CCW mode, and the incident light field remains in its initial fiber in the condition of the resonator-atom system operating in the undercoupled regime, $\kappa_{e x 1}, \kappa_{e x 2} \ll \Gamma_{+}$. Overall, the device realizes an optical circulator that routes light from the input port $P_{i}$ to the adjacent output port $P_{i+1}$ with $i \in\{1,2,3,4\}$ (see Figure 3).

In the experiment, the transmissions $T_{i j}$ to all output ports $P_{j}$ when sending a weak coherent probe field into the four different input ports $P_{i}$ have been measured [19]. And the performance of the circulator can be quantified with the fidelity $\mathcal{F}$ and the average photon survival probability $\eta$. The fidelity is evaluated as the overlap of the renormalized transmission matrix $\tilde{T}=\left(T_{i j} / \eta_{i}\right)$ with the one expected for the ideal circulator, $T^{\mathrm{id}}$. Here, $\eta_{i}=\sum_{k} T_{i, k}$ is the survival probability of a photon entering port $P_{i}$. Thus, the average operation fidelity of the circulator is [19]

$$
\mathcal{F}=\frac{\operatorname{Tr}\left[\tilde{T} T^{\mathrm{id}, T}\right]}{\operatorname{Tr}\left[T^{\mathrm{id}} T^{\mathrm{id}, T}\right]},
$$

giving the probability of the correct circulator operation average over various inputs. The minimum fidelity is $\mathcal{F}=0$, whereas $\mathcal{F}=1$ is reached for ideal operation. The experimental results show an optimum circulator performance for $\kappa_{\text {tot }} / 2 \kappa_{i}=2.2$, where $\mathcal{F}=0.72 \pm 0.03$ and, at the same time, $\eta=0.73 \pm 0.04$.

Furthermore, the circulator performance can also be quantified by the isolations [19].

$$
I_{i}=10 \log \left(T_{i, i+1} / T_{i+1, i}\right) .
$$

For the optimum working point, it achieves $\left\{I_{i}\right\}=\{10.9 \pm 2.5,6.8 \pm 1.3,4.7 \pm$ $0.7,5.4 \pm 1.1\} \mathrm{dB}$ and an average insertion loss of $-10 \log \eta=1.4 \mathrm{~dB}$ [19].

Note that when the atom is prepared in the opposite Zeeman ground state, $F=$ $3, m_{F}=-3$, the operation direction of the circulator is reversed.

The type-III single-photon isolator is based on a microring resonator coupling to a QD and a nearby waveguide [17]. In the approach, the silicon microring resonator in which light is tightly transversely confined has an exceptionally strong evanescent e-field and a near-unity OC surrounding the whole outside and inside walls of the resonator. By initializing a quantum dot (QD) in a specific spin ground state or using the optical Stark control, a broadband single-photon isolation over several gigahertz is achieved.

The QD-resonator system consists of a silicon waveguide, a silicon microring resonator, and a single negatively charged quantum dot (QD). Numerical 
simulations using the finite-difference time-domain (FDTD) method are performed to calculate the properties of the resonator. At the resonant wavelength $\lambda_{c} \sim$ $1.556 \mu \mathrm{m}$, the intrinsic quality factor $Q_{\text {in }}$ is about $3.9 \times 10^{4}$, and the mode volume $V_{m}$ is about $1.55 \mu \mathrm{m}^{3}$. The corresponding resonance frequency and the intrinsic decay rate are $\omega_{c} / 2 \pi \approx 192.67 \mathrm{THz}$ and $\kappa_{i} / 2 \pi \approx 4.94 \mathrm{GHz}$, respectively, yielding a total decay rate of $\kappa=\kappa_{e x}+\kappa_{i} \approx 2 \pi \times 9.88 \mathrm{GHz}$, where WGMs decay into the waveguide at the rate $\kappa_{e x}$ and $\kappa_{e x}=\kappa_{i}$ at resonance.

When the light enters the waveguide from port $P_{1}\left(P_{2}\right)$ with transverse electric mode and excites the CCW (CW) WGMs, the evanescent fields of interest circulating around the sidewalls of the resonator are tightly confined in the transverse direction as a transverse magnetic mode. The e-field distribution and the OC of the microring resonator are numerically investigated by FDTD simulation. For a TE mode incident to port $P_{1}\left(P_{2}\right)$, the intensity difference distribution $\mathcal{D}$ is shown in Figure $6 \mathbf{a}-\mathbf{c}$. When the light enters the waveguide from port $P_{1}$, the outer (inner) evanescent field of the WGM is $\sigma^{+}-\left(\sigma^{-}\right)$polarized, indicated by $\mathcal{C} \approx-1(1)$, as shown in Figure $6 \mathbf{b}$. For the light incident to port $P_{2}$, the polarization of the evanescent field is reversed, as shown in Figure 6d. Note that in this resonator, $|\mathcal{C}|>0.99$ from the surface of the outside wall to a position $280 \mathrm{~nm}$ away in the radial direction [17]. This large chiral area greatly relaxes the requirement for precisely positioning a QD. Importantly, the intensities of the evanescent fields near the outside wall are almost equal to that in the middle of the resonator, and they are still strong even at a position tens of nanometers away from the surface. These
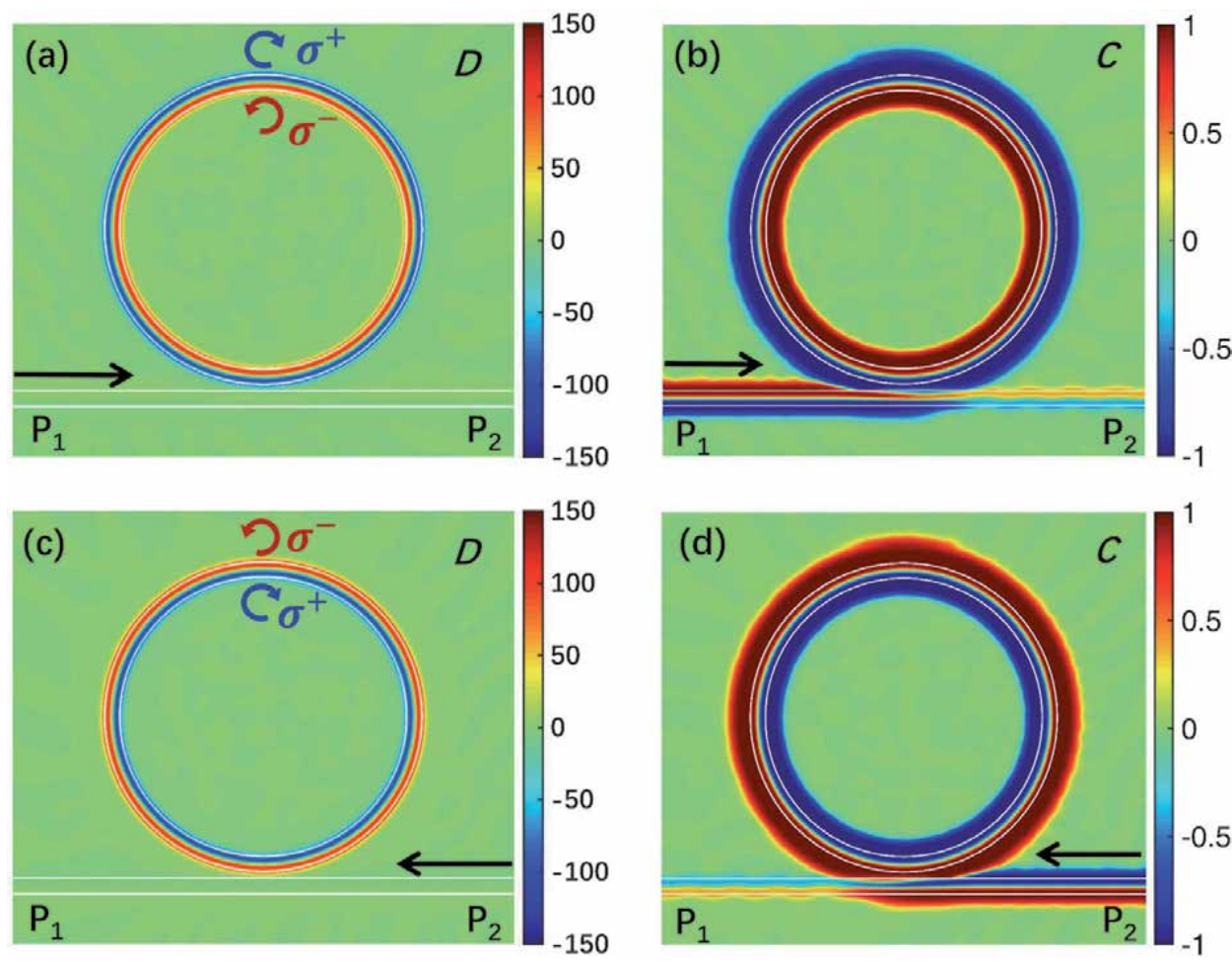

Figure 6.

$(a, c)$ Intensity difference $\mathcal{D}$ and $(b, d)$ optical chirality $\mathcal{C}$ for light with $\lambda=1.556 \mu \mathrm{m}$. Here, to clearly show the chiral e-fields in the vicinity of the resonator and wipe off the negligibly weak background, we use the definition for $\mathcal{C}=\left(\left|\mathbf{E}(\mathbf{r}) \cdot \hat{\sigma}^{-}\right|^{2}-\left|\mathbf{E}(\mathbf{r}) \cdot \hat{\sigma}^{+}\right|^{2}\right) /\left(|\mathbf{E}(\mathbf{r})|^{2}+\varrho\right)$, where we introduce a small bias, $\varrho=\max \left\{|\mathbf{E}(\mathbf{r})|^{2}\right\} \times$ $10^{-4}$, in the denominator. Light incident to port $P_{1}(a, b)$ and port $P_{2}(c, d)$. White lines are for the waveguide boundaries. Reproduced with permission from [17]. 
features of the resonator allow a strong chiral coupling between a nearby QD and the resonator.

As seen in Figure 7, a negatively charged QD is doped near the outside wall of the resonator. It has two energy-degenerate transitions at $\lambda_{q} \sim 1.556 \mu \mathrm{m}$, driven by a circularly polarized e-field, as seen in Figure 8a. It can be an InAs self-assembled QD grown on silicon dioxide/silicon substrates [29], with two electronic spin ground states $| \pm 1 / 2\rangle$ and two optically excited states $| \pm 3 / 2\rangle$.

By initializing the QD in a specific spin ground state or shifting the transition energy with the optical Stark effect (OSE), chiral QD-resonator interaction can be achieved. As shown in Figure 8b, by applying a magnetic field along the direction perpendicular to the growth direction of the QD, the spin-flip Raman transitions are enabled and can couple to linearly polarized e-fields. In this case, the spin ground state $|1 / 2\rangle$ or $|-1 / 2\rangle$ can be selectively prepared with a near-unity possibility $[27,30]$. When the spin ground state, e.g., $|1 / 2\rangle$, is populated and the magnetic field is switched off, the QD can be treated as a two-level system with a dipole moment coupling only with a $\sigma^{+}$-polarized e-field, as shown in Figure 8c. The second method involves all-optical control of the QD via the optical Stark control. The polarization-selective transition, $|1 / 2\rangle \leftrightarrow|3 / 2\rangle$ or $|-1 / 2\rangle \leftrightarrow|-3 / 2\rangle$, can also be tuned to have different energies by inducing a large optical Start shift with a large detuned circularly polarized light $[31,32]$. As shown in Figure 8d, the $\sigma^{+}$-polarized transition is shifted by $\sigma^{+}$-polarized classical light to be on resonance with the CCW mode, while the $\sigma^{-}$-polarized transition of the QD decouples to the resonator due to a large detuning $\Delta_{-}=\Delta_{c}+2 \Delta_{O S E}$, where $\Delta_{c}$ is the detuning of resonator's resonance and $\Delta_{O S E}$ is the detuning which resulted from the OSE. In this case, the QD can also be treated as a two-level system with a $\sigma^{+}$-polarized transition.

After QD spin ground state preparation, the QD strongly couples to the CCW mode with large strength $g_{+}$but decouples from the CW mode with a much smaller strength $g_{-}$. Note that the OSE-based method allows an all-optical operation. In fabrication, the QD can be engineered to have various resonance wavelengths, dipole moments, and decoherence rates. Self-assembled quantum dots can be engineered to possess a transition at $1.556 \mu \mathrm{m}$, and their dipole moment can vary

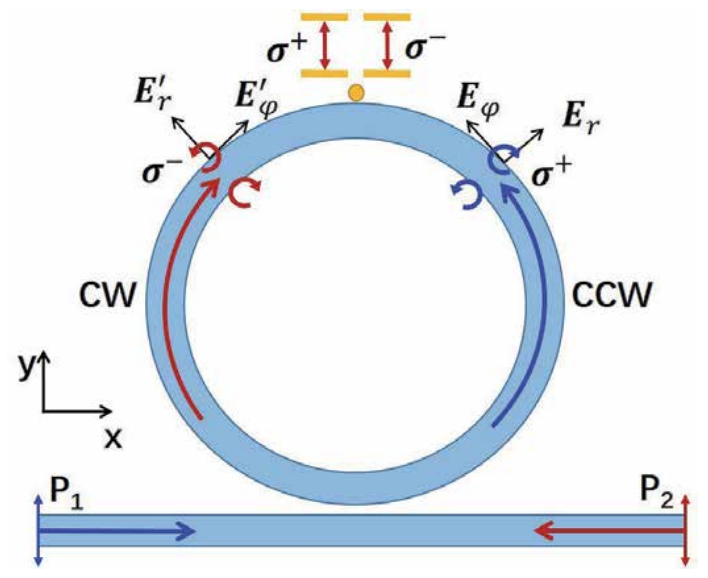

Figure 7.

Schematic of the single-photon isolation based on a microring resonator. The silicon resonator couples to a nearby silicon waveguide with refractive index $n=3.48$ and a single negatively charged $Q D$. The resonator and the waveguide are $0.44 \mu \mathrm{m}$ wide and $0.22 \mu \mathrm{m}$ thick. The resonator has a $4.22 \mu \mathrm{m}$ radius. The light incident to port $P_{1}\left(P_{2}\right)$ drives the counterclockwise (CCW) [clockwise (CW)] WGM. The polarization of the evanescent field of the CCW mode is $\sigma^{+}-\left(\sigma^{-}\right)$polarized near the whole outside (inside) wall, while that for the CW mode is $\sigma^{-}-\left(\sigma^{+}-\right)$polarized. After initialization for the $Q D$, it is treated as a two-level system with $\sigma^{+}$-polarized transition. Reproduced with permission from [17]. 

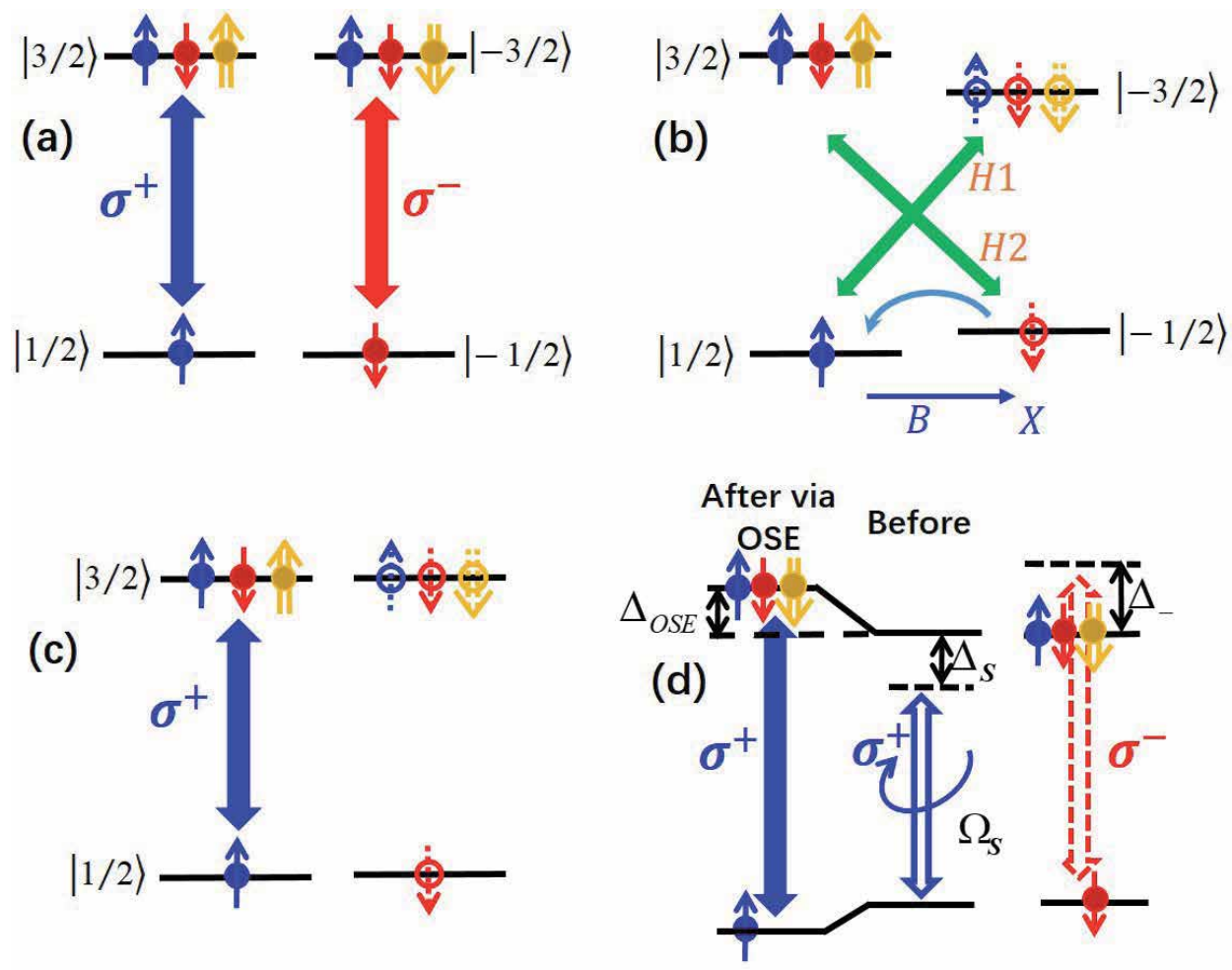

Figure 8.

Initialization of a negatively charged $Q D$ including two methods: Coherent population trapping $(a-c)$ and optical Stark effect $(d)$. (a) Four-level configuration of an electron spin in a single negatively charged $Q D .(b)$ Four-level configuration with dipole-allowed transitions, enabled by a magnetic field along the $X$ direction. (c) The Trion system which has been pumped with linearly polarized light at the magnetic field can be treated as a two-level system only with $\sigma^{+}$-polarized light excitation at zero magnetic field. (d) A $\sigma^{+}$-polarized classical light $\Omega_{s}$ with a detuning $\Delta_{s}$ from the $\sigma^{+}$-polarized transition $|1 / 2\rangle \leftrightarrow|3 / 2\rangle$ is applied to shift the transition energy by $\Delta_{O S E} \propto \Omega_{s}^{2} / \Delta_{S}$. The $\sigma^{-}$-polarized CW mode decouples from the QD because it is detuned by $\Delta_{-}$from the relevant transition $|-1 / 2\rangle \leftrightarrow|-3 / 2\rangle$. Figures are reproduced with permission from [17] and are slightly modified.

from a few Debye to 40 Debye [33]. Here, we choose the resonance wavelength $\lambda_{q} \approx 1.556 \mu \mathrm{m}, \omega_{q}=\omega_{c}$, and the dipole moment $|\mathbf{d}|=30$ Debye, yielding a spontaneous emission rate $\gamma_{q}=|\mathbf{d}|^{2} \omega_{q}^{2} / 3 \pi \varepsilon_{0} \hbar c^{3}=2 \pi \times 11.88 \mathrm{MHz}$. The strength of the zero-point fluctuation of this mode is $\left|\mathbf{E}_{0}\right|=\sqrt{\hbar \omega_{c} /\left(2 \varepsilon_{0} V_{m}\right) \approx 6.82 \times 10^{4} \mathrm{~V} / \mathrm{m}}$, where $\varepsilon_{0}$ is the vacuum permittivity and $\hbar$ is the Planck constant. Correspondingly, the QD-resonator coupling strength $g=\mathbf{d} \cdot \mathbf{E}_{0} / \hbar \approx 2 \pi \times 10.29 \mathrm{GHz}$. And asymmetry coupling strength $\left|g_{+}\right|=\alpha g$ and $\left|g_{-}\right|=\beta g$, where $\alpha=\sqrt{(1-\mathcal{C}) / 2}$ and $\beta=$ $\sqrt{(1+\mathcal{C}) / 2}$ [17]. As a consequence, the QD-resonator system is chiral and subsequently achieves the optical isolator at the single-photon level.

The steady-state forward (backward) transition amplitude $t_{+}\left(t_{-}\right)$, corresponding to the left-handed (right-handed) input, is derived by using the single-photon scattering method [17, 24, 34]

$$
t_{+}=\frac{\tilde{\Delta}_{c}\left[\tilde{\Delta}_{c} \tilde{\Delta}_{q}-G^{2}\right]+\tilde{\Delta}_{q} \kappa_{e x}^{2}-g_{+}^{*} g_{-} h-g_{+} g_{-}^{*} h^{*}-\tilde{\Delta}_{q}|h|^{2}+i\left(\left|g_{-}\right|^{2}-\left|g_{+}\right|^{2}\right) \kappa_{e x}}{\left(\tilde{\Delta}_{c}+i \kappa_{e x}\right)\left[\tilde{\Delta}_{q}\left(\tilde{\Delta}_{c}+i \kappa_{e x}\right)-G^{2}\right]-g_{+}^{*} g_{-} h-g_{+} g_{-}^{*} h^{*}-\tilde{\Delta}_{q}|h|^{2}},
$$




$$
t_{-}=\frac{\tilde{\Delta}_{c}\left[\tilde{\Delta}_{c} \tilde{\Delta}_{q}-G^{2}\right]+\tilde{\Delta}_{q} \kappa_{e x}^{2}-g_{-}^{*} g_{+} h-g_{-} g_{+}^{*} h^{*}-\tilde{\Delta}_{q}|h|^{2}+i\left(\left|g_{+}\right|^{2}-\left|g_{-}\right|^{2}\right) \kappa_{e x}}{\left(\tilde{\Delta}_{c}+i \kappa_{e x}\right)\left[\tilde{\Delta}_{q}\left(\tilde{\Delta}_{c}+i \kappa_{e x}\right)-G^{2}\right]-g_{-}^{*} g_{+} h-g_{-} g_{+}^{*} h^{*}-\tilde{\Delta}_{q}|h|^{2}},
$$

where $\tilde{\Delta}_{c}=\omega-\omega_{c}+i \kappa_{i}, \tilde{\Delta}_{q}=\omega-\omega_{q}+i \gamma_{q}$, and $G^{2}=\left|g_{+}\right|^{2}+\left|g_{-}\right|^{2}$. The detuning is defined as $\Delta_{c}=\omega-\omega_{c}$ and $\omega_{c}=\omega_{q}$ is assumed. In this device, $|\mathcal{C}|=$ 0.99 and $|h| \ll \kappa_{i}$, the transmissions, $T_{ \pm}=\left|t_{ \pm}\right|^{2}$, are shown in Figure 9. In the absence of the backscattering, i.e., $h=0$, at $\Delta_{c}=0, T_{+} \approx 0.99$ and $T_{-} \approx 0$, yielding the insertion loss of $\mathcal{L}=-10 \log \left(T_{+}\right) \approx 0.04 \mathrm{~dB}$ and the isolation contrast $\Upsilon \approx 1$. Consequently, at vanishing detuning, the single-photon isolation is achieved with almost zero insert loss and near-unity isolation contrast. The nonreciprocal bandwidth is about $1.3 \kappa \approx 2 \pi \times 12.8 \mathrm{GHz}$, which is about two to three orders broader than those in $[16,18-20,35]$. It can be seen from Figure 9a that the nonreciprocal spectral window becomes narrower and narrower as the backscattering strength increases; for a relatively large backscattering $|h|=\kappa_{i}$, both the backward and forward transmissions only change very slightly, but for an extremely large backscattering $|h|=3 \kappa_{i}$, the nonreciprocal performance is much affected. As shown in Figure $9 \mathrm{~b}$, in the absence of backscattering, the isolation contrast is quite robust, decreasing slowly from 1 to 0.8 as the OC changes from -1 to -0.5 (blue solid curve), while the insertion loss increases almost linearly in this region.

This device can achieve optical isolation when oppositely propagating photons enter the system at the same time, avoiding the dynamic reciprocity problem [36].
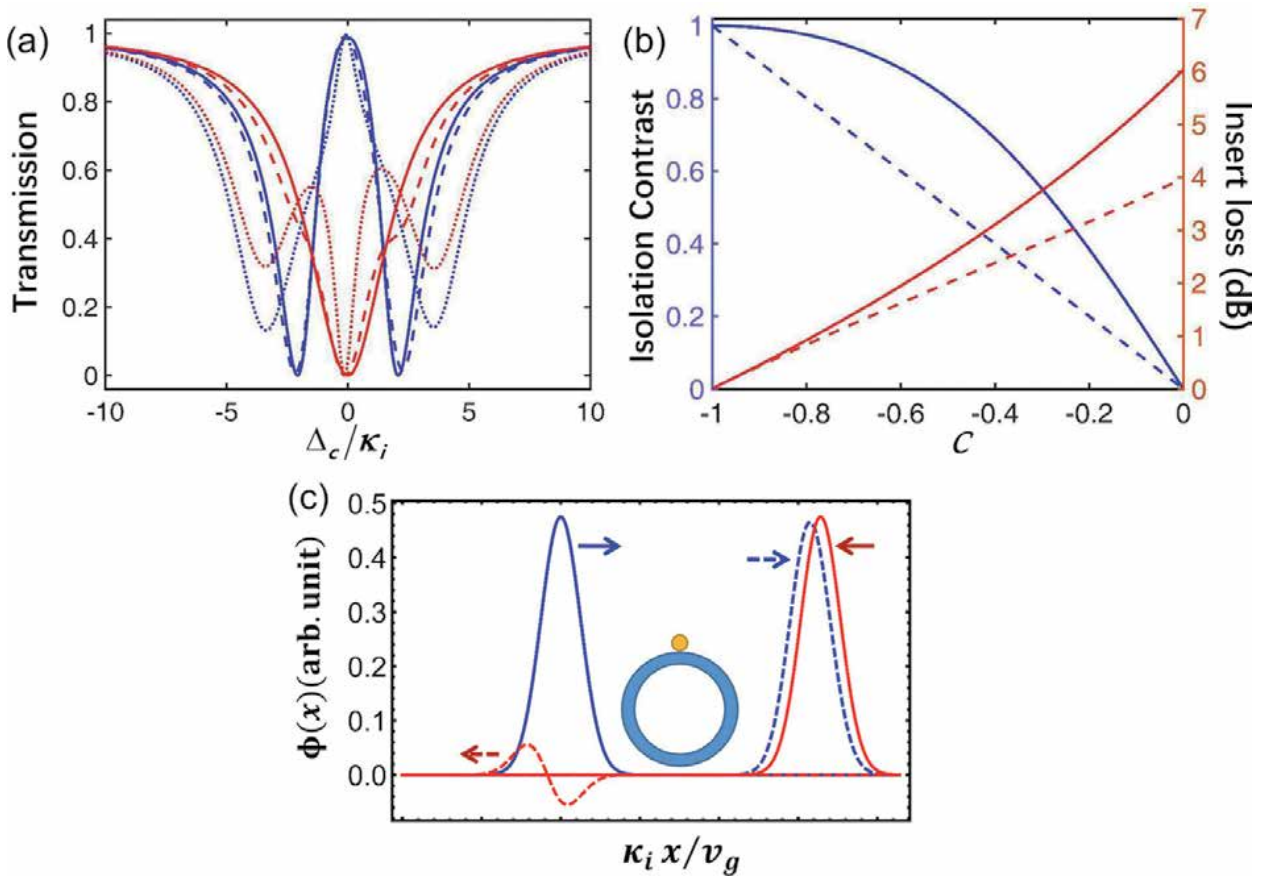

Figure 9.

(a) Steady-state transmissions for $|D|=0.99$. Blue (red) curves are for transmissions $T_{+}\left(T_{-}\right), h=o$ (solid curves) for $|h|=\kappa_{i}$ (dashed curves), and $|h|=3 \kappa_{\mathrm{i}}$ (dotted curves). (b) Blue curve is for isolation contrast, and red dashed curve is for insert loss as a function of the optical chirality D for $h=o$ (solid curves) and $|h|=\kappa_{i}$ (dashed curves). (c) Propagation of single-photon pulses incident to ports $P_{1}$ and $P_{2}$ simultaneously. Red thin (blue thick) curves are for the propagation of the right-moving (left-moving) single-photon pulses. Solid curves are for incident single-photon wave function, and dashed curves are for transmitted wave function. $|D|=1$ for simplicity. 
Numerical simulations for the propagation of single-photon wave packets incident to ports $P_{1}$ and $P_{2}$ simultaneously are performed by using wave-vector-space method [17]. The propagation of single-photon pulses in the system is shown in Figure 9c. At resonance, a right-moving single photon can pass through the system with transmission probability 0.98 , while that of a left-moving single photon is only 0.02 .

\section{Optical isolation via chiral cross-Kerr nonlinearity}

In a waveguide embedded with $N$-type atoms (see the upper waveguide in Figure 10a), the classical switching and coupling fields are applied to induce the phase shift $\phi$ and amplitude modulation $\xi$ of the probe field. The forward and backward amplitude transmissions $\xi_{f}$ and $\xi_{b}$ are sufficiently different after the probe field passes through the ensemble of atoms. Thus, the type-IV optical isolator is achieved.

$\mathrm{Rb}$ atoms are used to create the chiral XKerr nonlinearity. In the $|2\rangle \rightarrow|1\rangle$ transition, the $|2\rangle \rightarrow|3\rangle$ transition, and $|4\rangle \rightarrow|3\rangle$ transition of the $\mathrm{Rb}$ atoms with decay rates $\gamma_{21}, \gamma_{22}$, and $\gamma_{43}$, respectively, $\gamma_{21}=\gamma_{23}=\gamma_{43}=\gamma_{0}$ is assumed, and $\gamma_{0} \gg \Gamma$, where $\Gamma$ is the dephasing rates of both ground states $|1\rangle$ and $|3\rangle$ and $\gamma_{0}=$ $2 \pi \times 6 \mathrm{MHz}$. The XKerr nonlinearity can be efficiently induced between the probe and switching fields in the configuration and can be modified by the coupling laser. As shown in Figure 10b, the switching (coupling, probe) field drives the transition $|1\rangle \leftrightarrow|2\rangle(|3\rangle \leftrightarrow|2\rangle,|3\rangle \leftrightarrow|4\rangle)$ with a detuning $\Delta_{s}\left(\Delta_{c}, \Delta_{p}\right)$ in the absence of
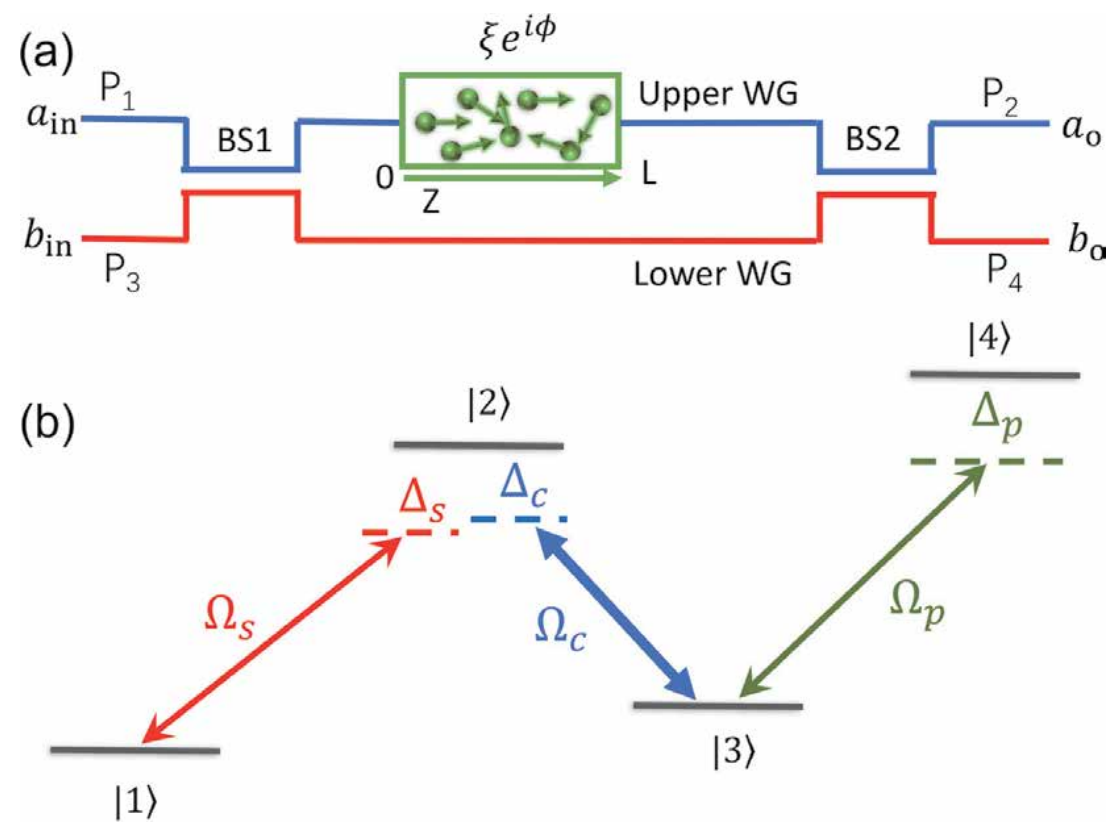

Figure 10.

(a) Schematic of the realization of optical isolator and circulator by using chiral cross-Kerr nonlinearity. To realize an optical isolator, we use only the upper waveguide (WG) embedded with a cloud of N-type atoms. The photon passing through the atoms suffers an amplitude transmission of $\xi$ and a phase shift $\phi$, which are dependent on its propagation direction. To achieve optical circulator, the lower waveguide is added to form a Mach-Zehnder interferometer with the upper one by using two beam splitters BS1 and BS2. (b) Energy-level diagram of $N$-type atoms. The switching (carrier frequency $\Omega_{\mathrm{s}}$ ), coupling $\left(\Omega_{c}\right)$, and probe $\left(\Omega_{p}\right)$ fields couple to transition $|1\rangle \leftrightarrow|2\rangle,|3\rangle \leftrightarrow|2\rangle$, and $|3\rangle \leftrightarrow|4\rangle$, with detunings $\Delta_{s}, \Delta_{c}$, and $\Delta_{p}$, respectively. Reproduced with permission from [20]. 
thermal motion. The switching (coupling, probe) laser beam has the carrier frequency $\Omega_{s}\left(\Omega_{c}, \Omega_{p}\right)$, corresponding to the wave vector $k_{s}\left(k_{c}, k_{p}\right)$. At room temperature, the inevitable random thermal motion of the $j$ th atom moving with velocity $v_{j}$ induces the "microscopic" Doppler shifts $k_{s} v_{j}, k_{c} v_{j}$, and $k_{p} v_{j}$ in the corresponding atomic transitions, respectively. The strength of the nonlinearity is strongly dependent on the effective detunings, and thus the Doppler shifts. As a result, these frequency shifts change the optical nonlinearity in a way strongly dependent on the propagation direction of the probe field with respect to the switching and coupling fields, leading to the chiral XKerr nonlinearity. Both the switching and coupling laser beams are left-moving and $k_{s} v_{j}=k_{c} v_{j}$ is assumed. Thus, the backward-moving (forward-moving) probe field "sees" the same (opposite) Doppler shift as the switching and coupling ones. Compared with the backward input case, where the Doppler broadening significantly reduces the total XKerr nonlinearity, the Doppler shift "seen" by the forward-moving probe field is partly compensated, and subsequently the nonlinearity remains large [20].

For a centimeter-scale medium, e.g., $L=2 \mathrm{~cm}$, the medium is absorptive, and the forward and backward transmissions are very different, as shown in Figure 11a. As the probe detuning increases, the forward transmission $T_{12}$ rapidly increases to 0.80 at $\Delta_{p}=35.6 \gamma_{0}$, corresponding to an insertion loss of $1 \mathrm{~dB}$. As a result of Doppler broadening, the backward transmission $T_{21}$ is much smaller than $T_{12}$, when $35.6 \gamma_{0}<\Delta_{p}<60.6 \gamma_{0}$. In this region, the insertion loss is smaller than $1 \mathrm{~dB}$, while the isolation ratio is larger than $15 \mathrm{~dB}$. The isolation ratio can be considerably improved with a large forward transmission by using a longer medium or, equivalently, increasing the density of the atoms. For $L=4 \mathrm{~cm}$, the isolation rate can reach more than $30 \mathrm{~dB}$ in the range of $50 \gamma_{0}<\Delta_{p}<60 \gamma_{0}$, yielding an isolation bandwidth of $2 \pi \times 60 \mathrm{MHz}$. At the same time, the insertion loss is less than $1 \mathrm{~dB}$. As a consequence, an isolation can be achieved by using this medium.

By carefully choosing the density and length of the atomic vapor, and properly arranging the switching and coupling fields, a phase shift difference, $\Delta \phi=\phi_{f}-\phi_{b}$, can approach $\pi$ with high transmissions $\xi_{f}$ and $\xi_{b}$. It can realize a four-port optical circulator by adding a lower waveguide to form a Mach-Zehnder interferometer (MZI), as seen in Figure 10a.

For a short medium $(L=3.33 \mathrm{~mm})$, the transmission of the forward- and backward-moving probes can be comparably high, but the phase shift $\phi_{b}$ is always small, specifically about $0.011 \pi$ at $\Delta_{p}=7.77 \gamma_{0}$, as shown in Figure 11b. In contrast, at the optimal point $\Delta_{p}^{\mathrm{opt}}=7.77 \gamma_{0}$, the difference of the phase shift, $\phi_{f}-\phi_{b}$, reaches
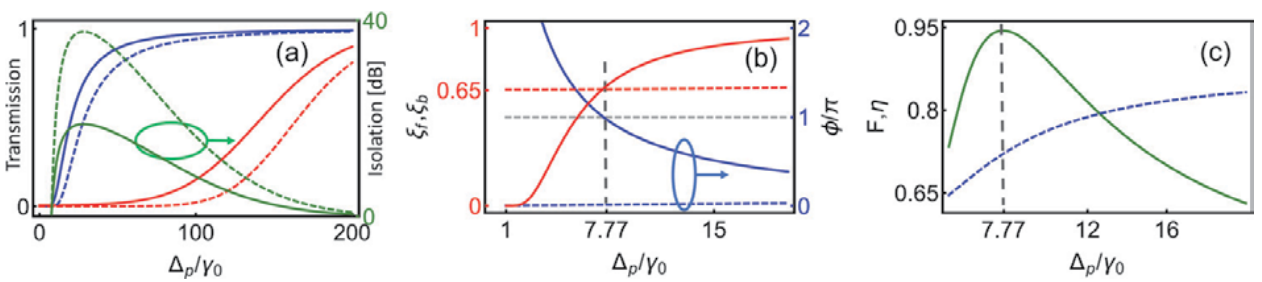

Figure 11.

(a) The transmission of the isolator for forward-moving (blue curves) and backward-moving (red curves) probe fields as a function of the probe detuning $\Delta_{\mathrm{p}}$. Green curves are for the isolations. Solid (dashed) curves are for the length of medium $\mathrm{L}=2(4) \mathrm{cm}$. (b) Amplitude transmissions (red curves) and phase shifts (blue curves) for forward-moving (solid curves) and backward-moving (dashed curves) probe fields as a function of $\Delta_{\mathrm{p}}$. (c) Green curves are for fidelities, and blue dashed curves are for average insertion loss as a function of $\Delta_{\mathrm{p}}$. The vertical black dashed lines in the two figures show the optimal detuning $\Delta_{p}^{\text {opt }}=7.77 \gamma_{0}$. The length of medium is $3.33 \mathrm{~mm}$. Other parameters $N_{a}=5 \times 10^{12} \mathrm{~cm}^{-3}, \Gamma_{3}=0.1 \gamma_{o}, \Omega_{c}=20 \gamma_{o}, \Omega_{s}=4 \gamma_{o}$, and $\delta=0$ are fixed. Reproduced with permission from [20]. 
the optimal value of $\pi$. At the same time, $\xi_{f}^{\text {opt }} \approx \xi_{b}^{\text {opt }} \approx 0.66$. As a result, a highperformance circulator can be realized by inserting this nonlinear medium into a MZI composed of unbalanced BSs. In the lower waveguide, a phase shift $\vartheta=0.01 \pi$ is added to compensate the phase shift of the backward-moving photon in the upper one. As shown in Figure 11c, when the detuning $\Delta_{p}$ varies from $6 \gamma_{0}$ to $20 \gamma_{0}$, the fidelity $\mathcal{F}$ first rises up rapidly, reaches the maximum 0.944 at $\Delta_{p}^{\mathrm{opt}}=7.77 \gamma_{0}$, and then decreases. At the same time, the average photon survival probability $\eta$ increases from 0.68 to 0.83 . Although the photons have a larger probability to survive at a large detuning, the fidelity is low. Thus, there is a trade-off between the fidelity and the survival probability. The circulator operating within the frequency range $6.6 \gamma_{0}<\Delta_{p}<9.7 \gamma_{0}$ can achieve a fidelity larger than 0.9 at the expense of $\eta>0.69$. The corresponding working window is about $2 \pi \times 20 \mathrm{MHz}$, and the average insertion loss is about $1.6 \mathrm{~dB}$. At the $\Delta_{p}^{\text {opt }}$, it obtains a fidelity $\mathcal{F}=0.944$ and a survival probability $\eta=0.72$, yielding an insertion loss of $1.42 \mathrm{~dB}$. The circulator can also be quantified by the isolations $\left\{\mathcal{F}_{i}\right\}=\{41.7,13.8,13.8,8.2\}$ dB with $i=$ $\{1,2,3,4\}$ (see Eq. (7)), implying nonreciprocal photon circulator along $1 \rightarrow 2 \rightarrow$ $3 \rightarrow 4 \rightarrow 1$.

The proposal can achieve the nonlinear optical isolation without dynamic reciprocity [36], because the XKerr nonlinearity itself is chiral and the isolation is based on linear equations [20]. According to this proposed method, the device that uses XKerr nonlinearity to achieve cavity-free optical isolator and circulator at ultralow light level has been demonstrated experimentally [21].

\section{Conclusions}

In this chapter, we introduce the optical chirality of light confined around nanophotonic structures and the chiral optical XKerr nonlinearity induced in atoms. Based on optical chirality, we propose single-photon isolators and circulators with chiral light-emitter interaction. These concepts have been demonstrated experimentally. Then we showed approaches to achieve an optical isolator and a circulator by using the chiral XKerr nonlinearity. All of these approaches can realize chipcomparable optical isolations with low insertion loss and high isolation performance. The methods also work at ultralow light level and even single-photon level. These optical isolators and circulators may pave the way for photon routing and information processing in a nonreciprocal way in integrated optical circuits and quantum networks.

\section{Acknowledgements}

K. X. thanks the support of the National Key R\&D Program of China (Grant No. 2017YFA0303703) and the National Natural Science Foundation of China (Grants Nos. 11874212 and 61435007). He also thanks his co-workers, in particular, Prof. Jason Twamley, Prof. Yong Zhang, Prof. Min Xiao, and Prof. Franco Nori for their essential contribution to the original works.

\section{Abbreviations}

OC 


$\begin{array}{ll}\text { QD } & \text { quantum dot } \\ \text { OSE } & \text { optical Stark effect } \\ \text { CCW } & \text { counterclockwise } \\ \text { CW } & \text { clockwise } \\ \text { FDTD } & \text { finite-difference time-domain } \\ \text { XKerr } & \text { cross-Kerr } \\ \text { MZI } & \text { Mach-Zehnder interferometer } \\ \text { BS } & \text { beam splitter }\end{array}$

\section{Author details}

Lei Tang ${ }^{1,2,3 * \dagger}$ and Keyu Xia ${ }^{1,2,3+}$

1 College of Engineering and Applied Sciences, Nanjing University, Nanjing, China

2 National Laboratory of Solid State Microstructures, Collaborative Innovation Center of Advanced Microstructures, Nanjing University, Nanjing, China

3 Jiangsu Key Laboratory of Artificial Functional Materials, and Key Laboratory of Intelligent Optical Sensing and Manipulation, Ministry of Education, Nanjing University, Nanjing, China

*Address all correspondence to: tanglei@smail.nju.edu.cn

$\uparrow$ These authors contributed equally.

\section{IntechOpen}

(C) 2019 The Author(s). Licensee IntechOpen. This chapter is distributed under the terms of the Creative Commons Attribution License (http://creativecommons.org/licenses/ by/3.0), which permits unrestricted use, distribution, and reproduction in any medium, provided the original work is properly cited. (c) BY 


\section{References}

[1] Cirac JI, Zoller P, Kimble HJ, Mabuchi H. Quantum state transfer and entanglement distribution among distant nodes in a quantum network. Physical Review Letters. 1997;78(16):3221-3224. DOI: 10.1103/PhysRevLett.78.3221

[2] Kimble HJ. The quantum internet. Nature. 2008;453(7198):1023-1030. DOI: $10.1038 /$ nature 07127

[3] Hacker B, Welte S, Rempe G, Ritter S. A photon-photon quantum gate based on a single atom in an optical resonator. Nature. 2016;536(7615): 193-196. DOI: $10.1038 /$ nature18592

[4] Riedinger R, Hong S, Norte RA, Slater JA, Shang J, Krause AG, et al. Non-classical correlations between single photons and phonons from a mechanical oscillator. Nature. 2016; 530(7590):313-316. DOI: 10.1038/ nature16536

[5] Sathyamoorthy SR, Tornberg L, Kockum AF, Baragiola BQ, Combes J, Wilson CM, et al. Quantum nondemolition detection of a propagating microwave photon. Physical Review Letters. 2014;112(9): 093601. DOI: 10.1103/PhysRevLett. 112.093601

[6] Bi L, Hu J, Jiang P, Kim DH, Dionne GF, Kimerling LC, et al. On-chip optical isolation in monolithically integrated non-reciprocal optical resonators. Nature Photonics. 2011; 5(12):758-762. DOI: $10.1038 /$ NPHOTON.2011.270

[7] Tien MC, Mizumoto T, Pintus P, Kromer H, Bowers JE. Silicon ring isolators with bonded nonreciprocal magneto-optic garnets. Optics Express. 2011;19(12):11740-11745. DOI: 10.1364/ OE.19.011740

[8] Fan L, Wang J, Varghese LT, Shen H, Niu B, Xuan Y, et al. An all-silicon passive optical diode. Science. 2012; 335(6067):447-450. DOI: 10.1126/ science. 1214383

[9] Peng B, Özdemir Ş K, Lei F, Monifi F, Gianfreda M, Long GL, et al. Parity-timesymmetric whispering-gallery microcavities. Nature Physics. 2014; 10(5):394-398. DOI: 10.1038/NPHYS2927

[10] Chang L, Jiang X, Hua S, Yang C, Wen J, Jiang L, et al. Parity-time symmetry and variable optical isolation in active-passive-coupled microresonators. Nature Photonics. 2014;8(7):524-529. DOI: 10.1038/ NPHOTON.2014.133

[11] Ruesink F, Miri MA, Alu A, Verhagen E. Nonreciprocity and magnetic-free isolation based on optomechanical interactions. Nature Communications. 2016;7:13662. DOI: 10.1038/ncomms13662

[12] Shen Z, Zhang YL, Chen Y, Zou CL, Xiao YF, Zou XB, et al. Experimental realization of optomechanically induced non-reciprocity. Nature Photonics. 2016;10(10):657-661. DOI: 10.1038/ NPHOTON.2016.161

[13] Fang K, Luo J, Metelmann A, Matheny MH, Marquardt F, Clerk AA, et al. Generalized non-reciprocity in an optomechanical circuit via synthetic magnetism and reservoir engineering. Nature Physics. 2017;13(5):465-471. DOI: $10.1038 /$ NPHYS4009

[14] Dong MX, Yu YC, Ye YH, Zhang WH, Li EZ, Zeng L, et al. Experimental realization of quantum non-reciprocity based on cold atomic ensembles. arXiv:1908.09242v1. 2019. https://arxiv.org/abs/1908.09242v1

[15] Lodahl P, Mahmoodian S, Stobbe S, Rauschenbeutel A, Schneeweiss P, Volz J, et al. Chiral quantum optics. Nature. 2017;541(7638):473-480. DOI: 10.1038/nature21037 
[16] Xia K, Lu G, Lin G, Cheng Y, Niu Y, Gong $\mathrm{S}$, et al. Reversible nonmagnetic single-photon isolation using unbalanced quantum coupling. Physical Review A. 2014;90(4):043802. DOI: 10.1103/PhysRevA.90.043802

[17] Tang L, Tang J, Zhang W, Lu G, Zhang H, Zhang Y, et al. On-chip chiral single-photon interface: Isolation and unidirectional emission. Physical Review A. 2019;99(4):043833. DOI: 10.1103/PhysRevA.99.043833

[18] Sayrin C, Junge C, Mitsch R, Albrecht B, O'Shea D, Schneeweiss P, et al. Nanophotonic optical isolator controlled by the internal state of cold atoms. Physical Review X. 2015;5(4): 041036. DOI: $10.1103 /$

PhysRevX.5.041036

[19] Scheucher M, Hilico A, Will E, Volz J, Rauschenbeutel A. Quantum optical circulator controlled by a single chirally coupled atom. Science. 2016; 354(6319):1577-1580. DOI: 10.1126/ science.aaj2118

[20] Xia K, Nori F, Xiao M. Cavity-free optical isolators and circulators using a chiral cross-Kerr nonlinearity. Physical Review Letters. 2018;121(20):203602. DOI: 10.1103/PhysRevLett.121.203602

[21] Li EZ, Ding DS, Yu YC, Dong MX, Zeng L, Zhang WH, et al. Experimental demonstration of cavity-free optical isolators and optical circulators. arXiv: 1908.07210v1. 2019. https://arxiv.org/ abs/1908.07210v1

[22] Mahmoodian S, Prindal-Nielsen K, Sölner I, Stobbe S, Lodahl P.

Engineering chiral light-matter interaction in photonic crystal waveguides with slow light. Optical Materials Express. 2017;7(1):43-51. DOI: 10.1364/OME.7.000043

[23] Shen Y, Bradford M, Shen JT. Single-photon diode by exploiting the photon polarization in a waveguide.
Physical Review Letters. 2011;107(17): 173902. DOI: 10.1103/PhysRevLett. 107.173902

[24] Shen JT, Fan S. Coherent photon transport from spontaneous emission in one-dimensional waveguides. Optics Letters. 2005;30(15):2001-2003. DOI: 10.1364/OL.30.002001

[25] Turchette QA, Hood CJ, Lange W, Mabuchi H, Kimble HJ. Measurement of conditional phase shifts for quantum logic. Physical Review Letters. 1995; 75(25):4710. DOI: 10.1103/PhysRevLett. 75.4710

[26] Junge C, O'shea D, Volz J, Rauschenbeutel A. Strong coupling between single atoms and nontransversal photons. Physical Review Letters. 2013;110(21):213604. DOI: 10.1103/PhysRevLett.110.213604

[27] Xu X, Sun B, Berman PR, Steel DG, Bracker AS, Gammon D, et al. Coherent population trapping of an electron spin in a single negatively charged quantum dot. Nature Physics. 2008;4(9):692-695. DOI: $10.1038 /$ nphys1054

[28] Vetsch E, Reitz D, Sagué G, Schmidt R, Dawkins ST, Rauschenbeutel A. Optical interface created by laser-cooled atoms trapped in the evanescent field surrounding an optical nanofiber. Physical Review Letters. 2010;104(20):203603. DOI: 10.1103/PhysRevLett.104.203603

[29] Choi BH, Park CM, Song SH, Son MH, Hwang SW, Ahn D, et al. Selective growth of InAs self-assembled quantum dots on nanopatterned $\mathrm{SiO}_{2} / \mathrm{Si}$ substrate. Applied Physics Letters. 2001; 78(10):1403-1405. DOI: 10.1063/ 1.1352049

[30] Atatüre M, Dreiser J, Badolato A, Högele A, Karrai K, Imamoglu A. Quantum-dot spin-state preparation with near-unity fidelity. Science. 2006; 312(5773):551-553. DOI: 10.1126/science. 1126074 
[31] Vora PM, Bracker AS, Carter SG, Sweeney TM, Kim M, Kim CS, et al. Spin-cavity interactions between a quantum dot molecule and a photonic crystal cavity. Nature Communications. 2015;6:7665. DOI: 10.1038/ncomms8665

[32] Yong CK, Horng J, Shen Y, Cai H, Wang A, Yang CS, et al. Biexcitonic optical stark effects in monolayer molybdenum diselenide. Nature Physics. 2018;14(11):1092-1096. DOI: 10.1038/s41567-018-0216-7

[33] Htoon H, Takagahara T, Kulik D, Baklenov O, Holmes AL Jr, Shih CK. Interplay of Rabi oscillations and quantum interference in semiconductor quantum dots. Physical Review Letters. 2002;88(8):087401. DOI: 10.1103/ PhysRevLett.88.087401

[34] Shen JT, Fan S. Theory of singlephoton transport in a single-mode waveguide. II. Coupling to a whispering-gallery resonator containing a two-level atom. Physical Review A. 2009;79(2):023838. DOI: 10.1103/ PhysRevA.79.023838

[35] Söllner I, Mahmoodian S, Hansen SL, Midolo L, Javadi A, Kiršanskej $G$, et al. Deterministic photon-emitter coupling in chiral photonic circuits. Nature Nanotechnology. 2015;10(9):775-778. DOI: 10.1038/NNANO.2015.159

[36] Shi Y, Yu Z, Fan S. Limitations of nonlinear optical isolators due to dynamic reciprocity. Nature Photonics. 2015;9(6):388-392. DOI: 10.1038/ NPHOTON.2015.79 
